\title{
WestVirginiaUniversity
}

THE RESEARCH REPOSITORY @ WVU

Graduate Theses, Dissertations, and Problem Reports

2009

\section{Exploring electron transfer in myoglobin-based transistors}

Debin Li

West Virginia University

Follow this and additional works at: https://researchrepository.wvu.edu/etd

\section{Recommended Citation}

Li, Debin, "Exploring electron transfer in myoglobin-based transistors" (2009). Graduate Theses,

Dissertations, and Problem Reports. 4491.

https://researchrepository.wvu.edu/etd/4491

This Dissertation is protected by copyright and/or related rights. It has been brought to you by the The Research Repository @ WVU with permission from the rights-holder(s). You are free to use this Dissertation in any way that is permitted by the copyright and related rights legislation that applies to your use. For other uses you must obtain permission from the rights-holder(s) directly, unless additional rights are indicated by a Creative Commons license in the record and/ or on the work itself. This Dissertation has been accepted for inclusion in WVU Graduate Theses, Dissertations, and Problem Reports collection by an authorized administrator of The Research Repository @ WVU.

For more information, please contact researchrepository@mail.wvu.edu. 


\title{
Exploring Electron Transfer \\ in Myoglobin-based Transistors
}

\author{
Debin Li \\ Dissertation submitted to the \\ Eberly College of Arts and Sciences \\ at West Virginia University \\ in partial fulfillment of the requirements \\ for the degree of \\ Doctor of Philosophy \\ in \\ Physics
}
David Lederman, Ph.D., Chair
Peter Gannett, Ph.D.
Larry Halliburton, Ph.D.
Thomas H. Myers, Ph.D.
Leonardo Golubovic, Ph.D.
Sergei Urazhdin, Ph.D.

Department of Physics

Morgantown, West Virginia

2009

Keywords: Myoglobin; Electron Transfer; Single Electron Transistor;

Break Junction; Electromigration 


\section{ABSTRACT \\ Exploring Electron Transfer \\ in Myoglobin-based Transistors}

Debin Li

Electron transfer in proteins is an issue of fundamental importance in biochemistry. This is especially true for proteins whose functionality relies on redox reactions, which by definition means that electron exchange must take place between the protein and another protein, a cofactor, or the external environment. An important example consists of proteins containing heme groups, where the iron ion in the protoporphyrin ring changes valence state depending on the whether an electron is accepted or given up by the protein. Much experimental work has been carried out since the late 1950s in order to develop an understanding of protein electron transfer mechanisms. This understanding is mainly based on the statistical average of protein ensembles measured by spectroscopic and electrochemical techniques.

Since the 1990s, electron transfer through individual molecules has been probed using electrochemical scanning tunneling microscopy (EC-STM) in aqueous solution at room temperature. Models that explain the behavior of electron transfer include the resonant tunneling model and two-step electron transfer model.

Recently, the fabrication of nanometer-gap electrodes by electromigration (break -junction) techniques has made it possible to study electron conductance through small single molecules that undergo redox reactions at cryogenic temperatures. By applying a bias voltage $V$ between the electrodes, as well as a gate voltage $V_{G}$ to the sample, the molecular energy levels can be probed and characteristic single-electron transistor (SET) behavior, such as a Coulomb blockade or a Kondo resonance, can be observed under the right conditions. This has created the possibility of making devices that exploit the unique electronic properties of organic and biomolecular compounds, such as their small size, stability, and dependence on conformation.

Considering the successful results in molecular SETs, an interesting question is whether similar devices can be fabricated using proteins. In principle, protein-based SETs could be observed at moderate cryogenic temperatures for heme proteins. Ideally, proteins with localized, well-defined energy levels are necessary.

In this work, the mechanism of electron transfer by myoglobin using nanometergap platinum electrodes was investigated. The electrodes were fabricated by breaking a small junction by electromigration at cryogenic temperatures. Apomyoglobin (myoglobin without the heme group) was used as a reference. The experimental results suggest single electron transport behavior is mediated by resonance of the electronic levels of the heme group in the myoglobin protein. This demonstrates that myoglobin across nanometer-gap electrodes could be utilized to fabricate single electron transistors. The orientation and conformation of myoglobin in the gap of electrodes may significantly affect the conductance of these devices. 


\section{Acknowledgements}

To the best of my knowledge, this is the first time electron transfer by a protein in a nanometer electronic device is studied at cryogenic temperatures. It took me a long time to solve several experimental and theoretical problems, and I will leave some problems unsolved. Anyway, it is my greatest pleasure to thank all the people who helped me bring this dissertation to fruition.

First, I thank my advisor, Dr. Lederman for guiding my research for about six years and his financial support even though there was no significant progress for several years. And I also thank him for his help with the calculation of the conductance due to the two-step electron transfer process.

I also thank Dr. Peter Gannett for his advice and comments on my research. He kindly taught me biochemistry. Dr. Sergei Urazhdin deserves special thanks as well. I thank him for giving me his advice and help on numerous occasions. He spent many hours with me, help me to solve many problem in electronics. And many thanks to Dr. Weng-Lee Lim in Dr. Urazhdin's group, who gave me a hand whenever I needed it.

I thank Dr. Halliburton for his encouragement during difficult times. I also thank Dr. Myers and Dr. Golubovic for their useful discussions. And many thanks to Dr. Jianhua Gu, Dr. Odille Myers, Eric Schires, Dr. Kolin Brown and all other people who helped me with my experiments when I needed them.

Finally, my warmest thanks go to my wife Yunqing Li. Without her love and support, this dissertation would not exist. 


\section{Table of Contents}

Abstract ........................................................ ii

Acknowledgements ........................................... iii

Table of Contents .............................................. iv

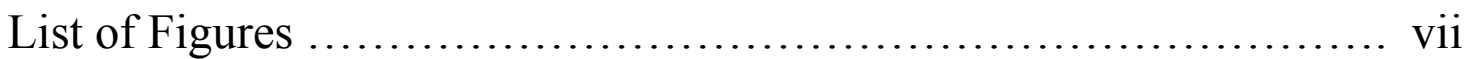

Chapter 1: Protein Electron Transfer ................................ 1

1.1 Introduction

1.2 Electron Transfer (ET)

1.3 Marcus Theory

1.4 Redox Potential

1.5 Cyclic Voltammetry (CV)

1.6 Electrochemical STM (EC-STM)

1.7 Some ET Models

1.8 Summary

Chapter 2: Basic Theories ............................................. 19

2.1 Overview

2.2 Inelastic Electron Tunneling

2.3 Elastic Electron Tunneling

2.4 Resonance Tunneling

2.5 Single Electron Transistor Theory

2.6 The Kondo Effect

2.7 Summary

Chapter 3: Device Fabrication

3.1 Introduction

3.2 Fabrication of Junctions

3.3 Breaking Junctions by Electromigration

3.4 Protein Deposition

3.5 Measurement Setup 
3.6 Summary

Chapter 4: Control Experiments in Blank Junctions

4.1 Introduction

4.2 The Origins of Conductance Peaks

4.3 The Pt Grains Produced During Electromigration

4.4 The Behavior of Metal Grains in the Gap of Electrodes

4.5 Localized Heating

4.6 Some Effective Methods

4.7 Summary

Chapter 5: Protein Immobilization

5.1 Introduction

5.2 Covalent Binding

5.3 Physical Immobilization

5.4 PBS Control Experiments

5.5 Summary

Chapter 6: Protein Vibrational Spectroscopy

64

6.1 Introduction

6.2 Vibrational State

6.3 Raman Spectroscopy

6.4 Raman Spectra for Myoglobin Proteins

6.5 Summary

Chapter 7: Electron Transfer by Apomyoglobin

7.1 Introduction

7.2 Electron Transfer Mechanism

7.3 One-dimensional Conductance

7.4 Some Results at $77 \mathrm{~K}$

7.5 Some Results at $\sim 5 \mathrm{~K}$

7.6 Summary

Chapter 8: Electron Transfer by Myoglobin 82

8.1 Introduction 
8.2 Cyclic Voltammetry (CV) for Mb Film

8.3 Electron Transfer Mechanism

8.4 The Charge State and Charging Energy

8.5 Some Results at $77 \mathrm{~K}$

8.6 Some Results at $\sim 5 \mathrm{~K}$

8.7 Summary

Chapter 9: Conclusions ........................................ 97

Bibliography ............................................. 99 


\section{List of Figures}

\section{Chapter 1}

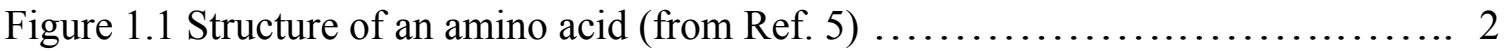

Figure 1.2 A covalent amide bond formed by two amino acids (from Ref. 5) .......... 3

Figure 1.3 Types of hydrogen bonds (from Ref. 5) ....................... 4

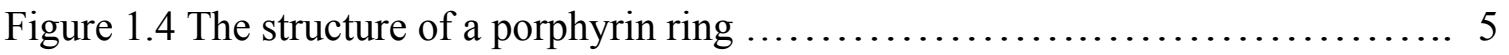

Figure 1.5 A: Detailed structure of the myoglobin protein. B: The heme group in oxymyoglobin, a porphyrin ring system complex with an iron ion (from Ref. 5) ...... 6

Figure 1.6 Splitting of the d orbitals in an octahedral field $\ldots \ldots \ldots \ldots \ldots \ldots \ldots \ldots \ldots .7$

Figure 1.7 Calculated orbital structure and relative energy of oxymyoglobin (from Ref. 9)

Figure 1.8 The electronic states of iron in myoglobin $\ldots \ldots \ldots \ldots \ldots \ldots \ldots \ldots \ldots . . . \ldots$

Figure 1.9 Energy diagram of the Marcus theory (from Ref. 2) $\ldots \ldots \ldots \ldots \ldots \ldots \ldots . \ldots 1$

Figure 1.10 Tao's results (from Ref. 3). (A) Schematic of Tao's STM experiment. (B) Energy diagram of the molecule. (C) The structure of the molecule. (D) The CV result

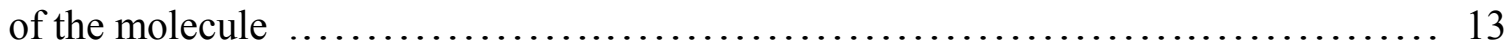

Figure 1.11 Park's results (from Ref. 13). (A) The structure of the molecule. (B) The CV result of the molecule. (C) Energy diagram of the molecule ................... 14

Figure 1.12 EC-STM configuration (from Ref. 15) $\ldots \ldots \ldots \ldots \ldots \ldots \ldots \ldots \ldots \ldots \ldots$

Figure 1.13 Energy diagram of EC-STM of redox molecule (from Ref. 16) ........... 16

Figure 1.14 Tunneling current vs. substrate potential in EC-STM (from Ref. 18) ...... 17

Figure 1.15 Energy diagram of the resonant tunneling model. (a) The redox level is above the Fermi levels of two electrodes. (b) The redox level is shifted to the Fermi levels of two electrodes by the gate potential $\ldots \ldots \ldots \ldots \ldots \ldots \ldots \ldots \ldots \ldots \ldots \ldots \ldots$

\section{Chapter 2}

Figure 2.1 Diagram of the elastic and inelastic electron tunneling (revised from Ref. 30) 
Figure 2.2 An electron with energy $E$ tunnels through a rectangular barrier 22

Figure 2.3 An electron with energy $E$ tunnels through a potential well .... 24

Figure 2.4 (a) Double barriers surrounding a well (b) Its transmission coefficient $T(\varepsilon)$ vs. $\varepsilon=E / V_{2}$ for $V_{3}<E_{0}$ (the resonance energy). The curves shown by dotted, solid and dashed lines are for $V_{3} / V_{2}=-2,0$ and 0.4 , respectively (from Ref. 33) ........... 26

Figure 2.5 A well surrounding two potential barriers ........................ 27

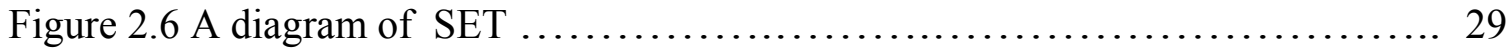

Figure 2.7 Energy diagram of single molecule level between two electrodes ......... 30

Figure 2.8 The energy diagram of a molecule with a single level .................... 33

Figure 2.9 Two-step electron transfer by a protein modeled as a quantum dot ......... 34

Figure 2.10 The calculation results for (a) with two-step process at $T=6 \mathrm{~K}, \delta E=25 \mathrm{mV}$, $E_{0}=25 \mathrm{mV}, C_{D}: C_{S}: C_{G}=12: 23.5: 1$, tunneling rates $\Gamma_{S}=\Gamma_{G}=10 \mathrm{GHz}$ (b) without twostep process at $T=6 \mathrm{~K}, \delta E=0 \mathrm{mV}, E_{0}=25 \mathrm{mV}, C_{D}: C_{S}: C_{G}=12: 23.5: 1$, tunneling rates

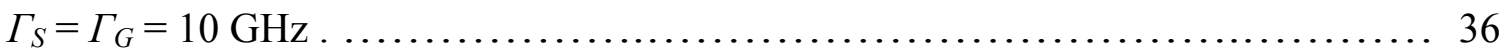

Figure 2.11 The Kondo effect in quantum dots. The red line show the Kondo effect for the odd number of electron confined in the dot. The blue line show the case that the

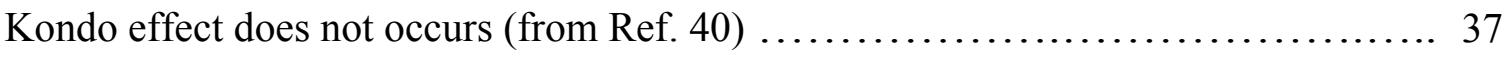

Figure 2.12 The Kondo effect from devices made using the shorter molecule, $\left[\mathrm{C}_{\mathrm{o}}\right.$ (tpy$\left.\mathrm{SH})_{2}\right]^{2+}$. Left graph show the temperature dependence of the Kondo peak. Right graph show the magnetic dependence of the Kondo peak (from Ref. 24) ................... 38

\section{Chapter 3}

Figure 3.1 There are 68 chips on 4 inch silicon wafer $\ldots \ldots \ldots \ldots \ldots \ldots \ldots \ldots \ldots \ldots . \ldots \ldots$ Figure 3.2 The sample holder in the cryostat. (A) in ARS, Helitran LT-3-110 open cycle cryostat. (B) in ARS, Displex DE-202 Closed Cycle cryostat .................. 40 Figure 3.3 A: An optical microscope image of a chip with 8 electrodes. B: An SEM micrograph of 6 junctions after e-beam lithography and lift-off $\ldots \ldots \ldots \ldots \ldots \ldots . \ldots 1$

Figure 3.4 Breaking junction by electromigration at cryogenic temperature. A: the gap after braking junction. B: I-V curve during breaking junction $\ldots \ldots \ldots \ldots \ldots \ldots \ldots, 42$ 
Figure 3.5 The two cryostat used for low temperature conductance measurements. (A) ARS, Helitran LT-3-110 open cycle cryostat (B) ARS, Displex DE-202 Closed Cycle

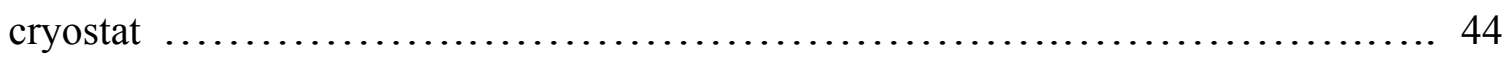

Figure 3.6 The program of breaking junction with feedback $\ldots \ldots \ldots \ldots \ldots \ldots \ldots \ldots .45$

Figure 3.7 The program of current vs. bias $\ldots \ldots \ldots \ldots \ldots \ldots \ldots \ldots \ldots \ldots \ldots \ldots \ldots \ldots$

\section{Chapter 4}

Figure 4.1 Two blank Pt junctions at $77 \mathrm{~K}$. (a) is a typical result (differential conductance $d I / d V_{\text {bias }}$ versus $\left.V_{\text {bias }}\right)$. The inset shows the current during the junctionbreaking process. (b) is the only one sample with conductance peaks

Figure 4.2 Measurements of blank Pt junctions at 5 6 K. (a) A sample that did not have peaks in the conductance vs. bias voltage. The inset shows the junction-breaking process. (b) One Pt junction with Coulomb diamonds, characteristic of SET behavior, was observed in the conductance as a function of gate and bias voltages. (c) Another sample with Coulomb diamonds, characteristic of SET behavior. (d) Conductance of blank Pt junction was broken by electromigration at $77 \mathrm{~K}$ and then cooled down to $5 \mathrm{~K}$....

Figure 4.3 Some results from Ref. 54. (a) shows when a single gold grain is left between the source and drain electrodes, the corresponding differential conductance plot has Coulomb diamonds (shown in (d)); (b) when the grain is attached to one of the leads by changing the deposition conditions, the differential conductance does not depend on the

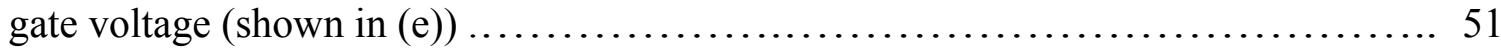

Figure 4.4 Differential conductance for four blank Au devices (at $250 \mathrm{mK}$ ) (from Ref. 47). (a) Coulomb blockade. (b) Superposition of a Coulomb blockade diamond and the Kondo effect. (c) Transition from Kondo effect to Coulomb diamond. (d) Kondo effect

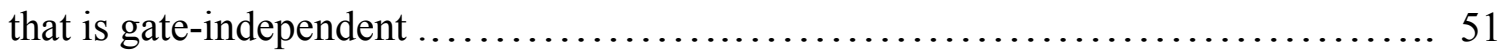
Figure 4.5 Simulation for two metal grains (from Ref. 53). (a) shows the system topology and energy decrements for each tunneling jump. (b) shows the differential conductance graph as a function of bias and gate voltage for the general case of a double-dot SET. The top graph is the result of a Monte Carlo simulation and the bottom graph is the result of direct numeric integration. (c) show all possible cases for a double-dot SET ....... 53 
Figure 4.6 I- $V$ characteristics of the two quantum dots system (from Ref. 58). The solid line for the system where the double-quantum-dot states are asymmetrically coupled to

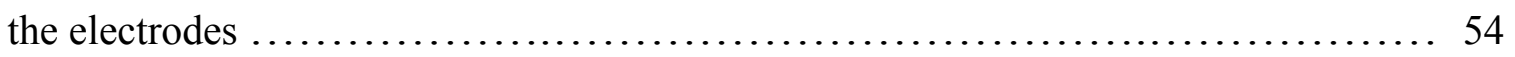
Figure 4.7 Two broken junctions. (A) Self-break (B)Break by electrostatic discharge .. 56

\section{Chapter 5}

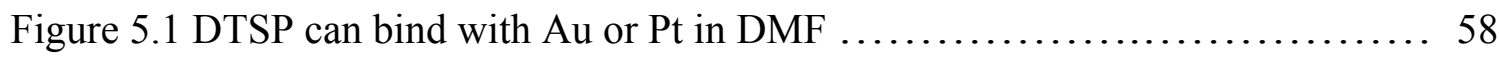

Figure 5.2 DTSP used to bind a protein to a Au or Pt surface $\ldots \ldots \ldots \ldots \ldots \ldots \ldots . . \ldots 8$

Figure 5.3 Two results of DTSP at $77 \mathrm{~K}$. Left graph shows there are two reproducible conductance peaks, measured at different times: $\mathrm{t} 1, \mathrm{t} 2$, $\mathrm{t} 3$. Right graph shows there is one

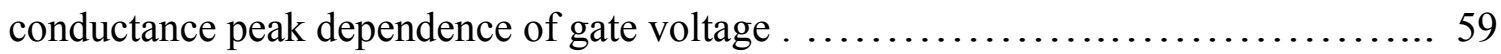
Figure 5.4 Two results of myoglobin with DTSP. (a) One result at $77 \mathrm{~K}$. (b) One result

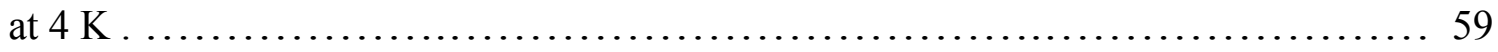

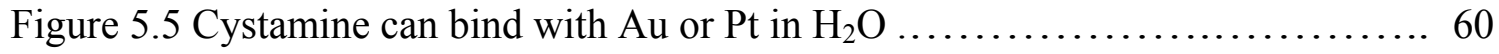
Figure 5.6 Two results from Cystamine bound with Pt at $77 \mathrm{~K}$. (a) One sample with one conductance peak at $77 \mathrm{~K}$. (b) Another sample at $77 \mathrm{~K}$ measured at different time: $\mathrm{t} 1, \mathrm{t} 2$, t3 60

Figure 5.7 One myoglobin sample without PBS measured at different time: $\mathrm{t} 1, \mathrm{t} 2$, $\mathrm{t} 3$ and t4

Figure 5.8 Two results from PBS at $77 \mathrm{~K}$. (a) One sample without conductance peak at $77 \mathrm{~K}$. (b) One samples had a conductance step or peak at $77 \mathrm{~K}$, Measured at different time: $\mathrm{t} 1, \mathrm{t} 2, \mathrm{t} 3$. 63

\section{Chapter 6}

Figure 6.1 Equivalent diatomic molecule $\ldots \ldots \ldots \ldots \ldots \ldots \ldots \ldots \ldots \ldots \ldots \ldots \ldots$

Figure 6.2 The vibrational levels of a diatomic molecule $\ldots \ldots \ldots \ldots \ldots \ldots \ldots \ldots .66$

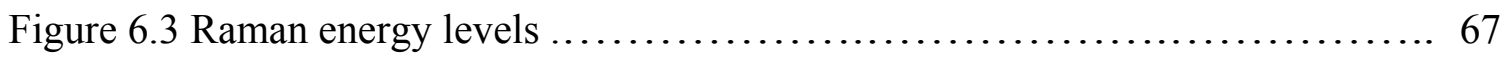

Figure 6.4 Vibronic transitions diagram based on the Franck-Condon principle (from

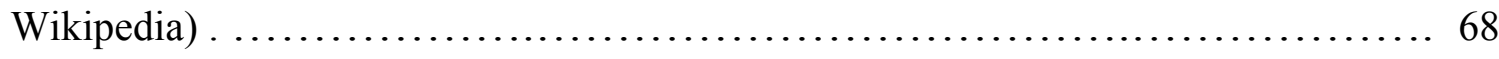


Figure 6.5 Resonance Raman spectra for aqueous metMb a and $\mathrm{Mb}$ film $\mathrm{b}$ in (a) $\lambda_{e x}=514.5 \mathrm{~nm}$ and (b) $\lambda_{e x}=406.7 \mathrm{~nm}$ (from Ref. 75) . .................... 69 Figure 6.6 Background checking. (a) $\mathrm{Si} / \mathrm{SiO} 2$ substrate. (b) Pt thin film on $\mathrm{Si} / \mathrm{SiO} 2$ substrate. (c) Dry PBS on $\mathrm{Si} / \mathrm{SiO} 2 / \mathrm{Pt}$ substrate. (d) Dry PBS on $\mathrm{Si} / \mathrm{SiO} 2 / \mathrm{Pt}$ substrata at

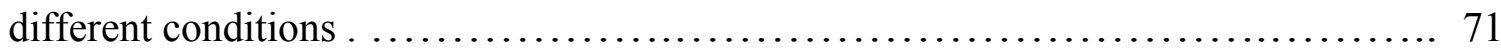

Figure 6.7 Raman spectroscopy for apomyoglobin. The black line shows the Raman spectra (1)(before cooling), the red line shows the Raman spectra (2)(after cooling) ... 72 Figure 6.8 Raman spectroscopy for myoglobin. The black line shows the Raman spectra (1)(before cooling). The red line shows the Raman spectra (2) (after cooling). The green line shows the Raman spectra (3)(drop PBS again after cooling down). The pink line shows the Raman spectra (4)(Mb in PBS and never cool down) ................... 72 Figure 6.9 The heme structural diagrams of (a) Mb in PBS; (b) dry Mb film (revised from Ref. 75) 73

\section{Chapter 7}

Figure 7.1 The possible mechanism for electron resonant tunneling through

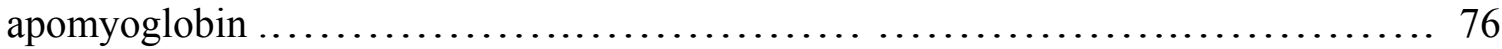

Figure 7.2 One apomyoglobin sample at $77 \mathrm{~K}$. (a) shows $I-V$ characteristics during the junction-breaking process. (b) shows that this junction was broken four times until steps in the $I-V$ graph were found. (c) is the conductance measured at $77 \mathrm{~K}$ with zero gate voltage which shows one conductance peak at $V_{\text {bias }}=-15 \mathrm{mV}$. (d) shows the conductance features remained more or less constant as a function of $V_{\mathrm{G}}$. (e) shows how this conductance peak changed with temperature. (f) shows the amplitude of the peak is plotted as a function of $1 / T$ which yields a straight line fit. (g) shows that the conductance peak changes slightly with gate voltage after cooling down again. (h) illustrates that the background conductance changed after warming up to $150 \mathrm{~K}$ and cooling back down to $77 \mathrm{~K}$. The black curve (t1) shows the initial conductance, the red curve (t2) shows the conductance after cooling down and warning up, and the blue one (t3) shows the final conductance after applying $V_{G}=+9.0 \mathrm{~V} \ldots \ldots \ldots \ldots \ldots \ldots \ldots$ 
Figure 7.3 One apomyoglobin sample at $77 \mathrm{~K}$. (a) shows that some conductance peaks can be found after breaking the junction. (b) shows the dependence of there conductance

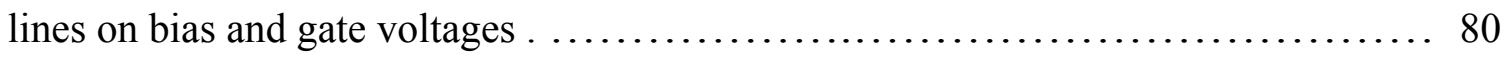
Figure 7.4 One apomyoglobin sample at $6 \mathrm{~K}$. (a) Reproducible conductance peaks after breaking the junction. (b) Conductance lines changed slightly with gate voltage. The red arrow indicates where one of the conductance peaks splits with gate voltage. Temperature dependence of these conductance lines .

\section{Chapter 8}

Figure 8.1 CV of (a) the $\mathrm{Mb}$ film electrode in a $\mathrm{pH} 7.4$ phosphate buffer and (b) a bare glassy carbon electrode in a buffer solution containing $5 \times 10^{-4} \mathrm{M}$ metMb. Scan rate $=0.1$ V/s (from Ref. 75)

Figure 8.2 (a) Protein measurement device. (b) There is an electrochemical potential $\mu$ $\left(\mathrm{Fe}^{3+} \rightarrow \mathrm{Fe}^{2+}\right)$ above the Fermi level. Just this level is drawn for simplicity (S: source, D:

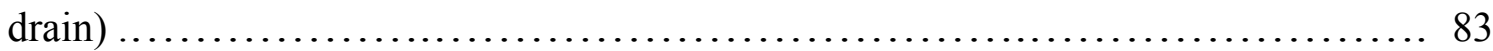

Figure $8.3 \mathrm{CV}$ at $2 \mathrm{~V} / \mathrm{s}$ in buffers containing $100 \mathrm{mM} \mathrm{NaBr}$ of $\mathrm{Mb}-\mathrm{DDAB}$ on $\mathrm{PG}$

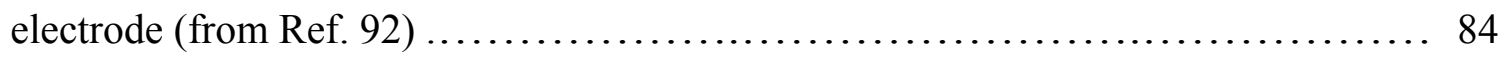

Figure 8.4 The electrochemical potential of protein. (a) for the isolated protein; (b) for the trapped protein .

Figure 8.5 Electron transport mechanism. (a) The redox level can be shifted to the Fermi level of the electrodes by the gate voltage. (b) Two-step electron transfer by protein ...86 Figure 8.6 (a) Calculated differential conductance $d I / d V$ as a function of gate and bias voltages without the two-step process for $T=6 \mathrm{~K}, E_{0}=4.72 \mathrm{mV}, C_{D}: C_{S}: C_{G}=24: 40: 1$, tunneling rates $\Gamma_{S}=\Gamma_{G}=10 \mathrm{GHz}$. (b) Calculated differential conductance $d I / d V$ as a function of gate and bias voltages with a two-step process for $T=6 \mathrm{~K}, \delta E=25 \mathrm{mV}, E_{0}=$ $25 \mathrm{mV}, C_{D}: C_{S}: C_{G}=12: 23.5: 1$, tunneling rates $\Gamma_{S}=\Gamma_{G}=10 \mathrm{GHz}$ 87

Figure 8.7 One myoglobin sample at 85 K. (a) Breaking junction process; (b) Some peaks or steps can be found in $d I / d V_{\text {bias }}$ as function of bias voltage, $V_{\text {gate }}=0 \mathrm{~V}$; (c)Differential conductance $d I / d V_{\text {bias }}$ as function of gate and bias voltage at $85 \sim 90 \mathrm{~K}$ (gray scale from

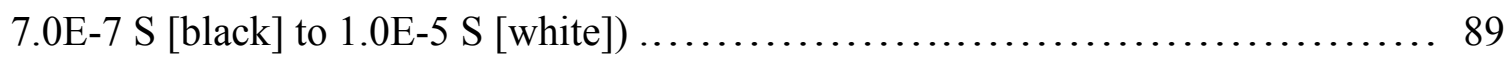


Figure 8.8 One of $\mathrm{Mb}$ samples at $77 \mathrm{~K}$ by $\mathrm{AC}$ measurement. (a) Some peaks can be found in $d I / d V_{\text {bias }}$ as function of bias voltage and gate voltage; (b)Differential conductance $d I / d V_{\text {bias }}$ as function of gate and bias voltage at $77 \mathrm{~K}$ (gray scale from -1.5

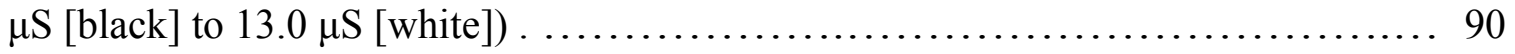

Figure 8.9 Enhanced zero bias conductance was observed in Mb samples (a) and apomyoglobin samples (b). The conductance of peaks are independence of gate voltage

Figure 8.10 One myoglobin sample at $6 \mathrm{~K}$. (a) The current suddenly increase a lot during breaking junction by electromigration; (b) Some conductance peaks can be found; (c) One the typical signature of single-electron transistor (gray scale from -1.5 $\mu \mathrm{S}$ [black] to $8.2 \mu \mathrm{S}$ [white]) ............................................................ 93

Figure 8.11 One myoglobin sample at $6 \mathrm{~K}$. (a) Differential conductance $d I / d V_{\text {bias }}$ as function of gate and bias voltage at $6 \mathrm{~K}$ (gray scale from $-0.27 \mu \mathrm{S}$ [black] to $1.6 \mu \mathrm{S}$ [white]); (b) Negative differential conductance from $-0.7 \mathrm{~V}$ to $-0.9 \mathrm{~V}$ (gate voltage); (c) The $I-V_{\text {bias }}$ curves change with gate voltage; (d) The $I-V_{\text {bias }}$ curves change with gate voltage 95 


\section{Chapter 1}

\section{Protein Electron Transfer}

\subsection{Introduction}

Electron transfer (ET) in proteins plays a crucial role in biological redox processes that are essential for life. Most significantly, respiration and photosynthesis processes rely entirely on electron transfer reactions in proteins. Identifying the fundamental mechanisms responsible for electron transfer in proteins over long distances is a problem that preoccupies physicists, chemists and biologists. As a result, much experimental work has been carried out since the late 1950s in order to develop an understanding of protein electron transfer mechanisms. ${ }^{1,2}$ Early experimental information was provided primarily by spectroscopic and electrochemical techniques. More recently, this has been investigated in single-molecules using electrochemical scanning tunneling microscopy (EC-STM) ${ }^{3}$ and this technique is now considered a mature field. ${ }^{4}$

In this chapter, the basic structure of proteins will be introduced and electron transfer will be explained in terms of the Marcus theory. Subsequently, cyclic voltammetry, an electrochemical technique that measures redox potential averaged over a large number of proteins, will be reviewed. Finally, EC-STM techniques and several models that deal with electron transfer by a redox center will be discussed.

\subsubsection{Amino Acids}

Amino acids, the building blocks of proteins, are composed of such elements as carbon, hydrogen, nitrogen, oxygen and sulfur. The general structure of an amino acid is shown in Figure 1.1..$^{5}$ All amino acids are built around a tetrahedral alpha $(\alpha)$ carbon $\left(\mathrm{C}_{\alpha}\right)$ which is covalently linked to four different groups: a hydrogen atom, an R group, an amino group and a carboxyl group. The $\mathrm{R}$ group determines the specific properties of the amino acid. Amino acids are joined together by peptide bonds between the carboxyl and amino groups of adjacent amino acid residues forming a linear chain as shown in Fig. 1.2. The bond is covalent in character and is so strong that it is practically never broken by thermal effects. 


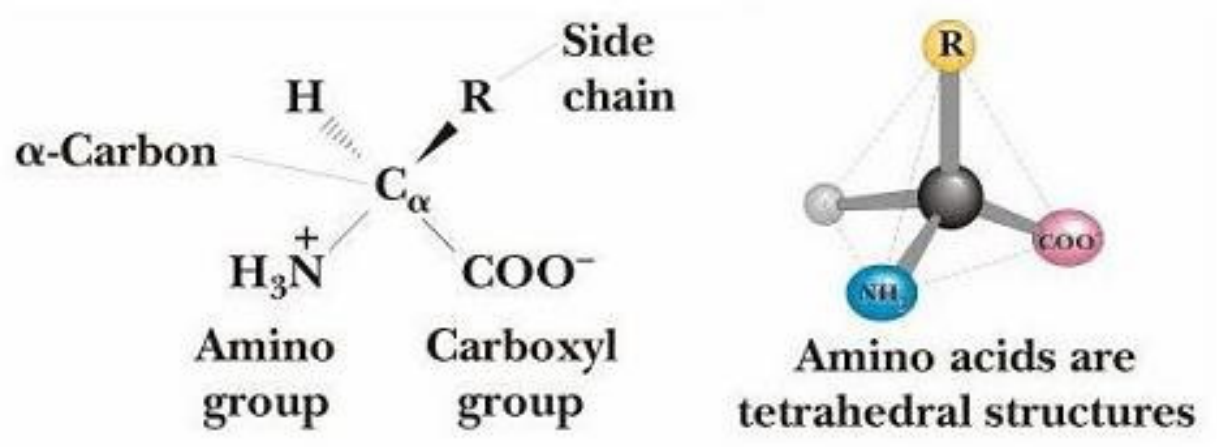

Figure 1.1 Structure of an amino acid (from Ref. 5).

Proteins consist of approximately 100 to 1000 amino acids which bind together to form a polypeptide chain. A polypeptide chain is formed from individual amino acids through peptide bonds, which consists of a backbone and two residues per amino acid. Proteins contain two terminal groups: the amino terminal $\left(-\mathrm{NH}_{3}{ }^{+}\right.$, denoted by $\left.-\mathrm{N}\right)$ and the carboxy terminal $\left(-\mathrm{COO}^{-}\right.$, denoted by $\left.-\mathrm{C}\right)$.

There are 20 common amino acids found in proteins ${ }^{5}$ which can be classified into four types based on the polarity of side chains: (1) Amino acids with nonpolar (hydrophobic) residues. These amino acids are less soluble in water than the polar amino acids. (2) Amino acids with uncharged polar residues. The polar R groups of these amino acids can hydrogen-bond with water and thus they are more soluble in water than those of group (1). (3) Amino acids with negatively charged (acidic) R groups. The members of this class possess a negative charge at $\mathrm{pH}=6-7$. (4) Amino acids with positively charged (basic) $\mathrm{R}$ groups. The basic amino acids have a positive charge at $\mathrm{pH}$ $=7$. 

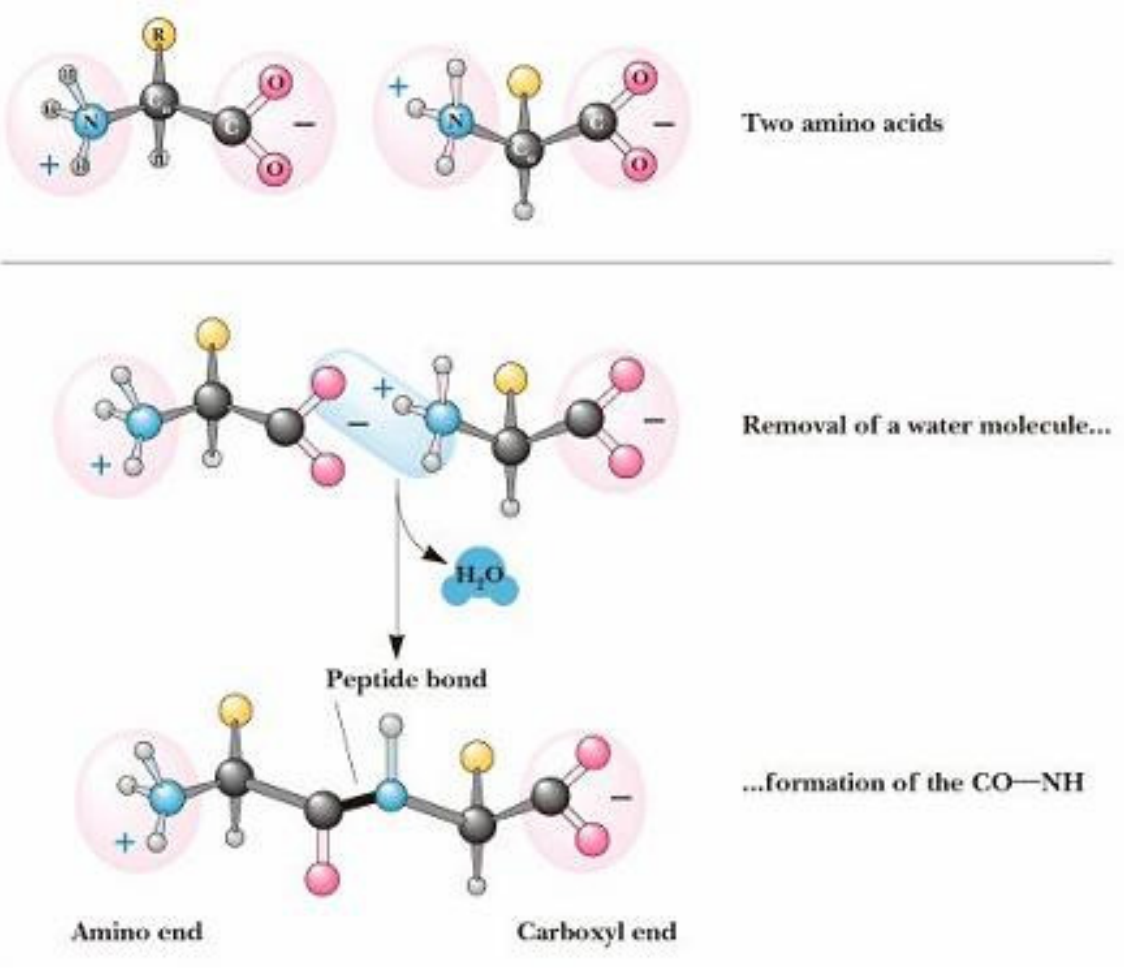

Figure 1.2 A covalent amide bond formed by two amino acids (from Ref. 5).

\subsubsection{Hydrogen Bond}

A hydrogen bond is a special type of dipole-dipole force between a hydrogen atom bonded to an electronegative atom (such as nitrogen or oxygen) and a second electronegative atom that serves as the hydrogen bond acceptor. This type of force always involves a hydrogen atom. A hydrogen atom bonded to an electronegative atom is a hydrogen bond donor. This electronegative atom is usually oxygen or nitrogen. An electronegative atom, such as oxygen or nitrogen, is a hydrogen bond acceptor, regardless of whether it is bonded to a hydrogen atom or not. Figure 1.3 shows a few types of hydrogen bonds and functional groups that serve as hydrogen bond donors and acceptors. This attractive force can occur between molecules or within different parts of a single molecule. The hydrogen bond is a very strong fixed dipole-dipole van der WaalsKeesom force, but weaker than covalent, ionic and metallic bonds. 


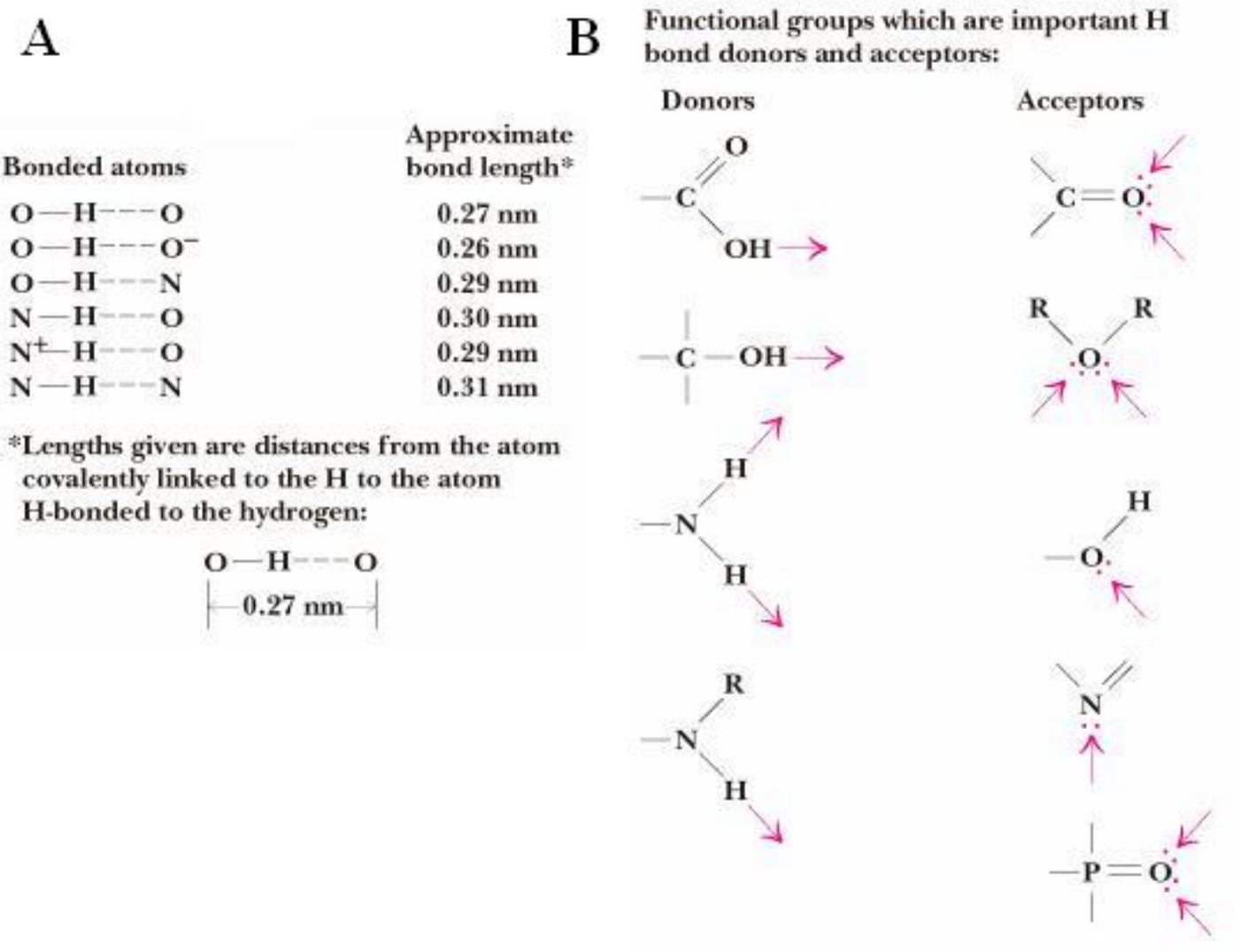

Figure 1.3 Types of hydrogen bonds (from Ref. 5).

\subsubsection{Protein Structure}

A protein's structure has four distinct aspects: (1) Primary structure - the amino acid sequence of the peptide chains. (2) Secondary structure - highly regular substructures (alpha helix and strands of beta sheet) which are locally defined, meaning that there can be many different secondary motifs present in one single protein molecule. (3) Tertiary structure - three-dimensional structure of a single protein molecule; a spatial arrangement of the secondary structures. (4) Quaternary structure - complex of several protein molecules or polypeptide chains.

\subsubsection{Proteins with Heme Group}


Proteins can be divided roughly into three classes on the basis of shape and solubility: globular proteins, fibrous proteins, and membrane proteins. Of particular interest are the globular proteins because essentially all enzymes (catalysts) are globular proteins. Proteins can be divided into functional proteins and structural proteins based on the functions they perform. Functional proteins are tailor-made for a specific job, such as storing energy or matter (oxygen) or catalyzing a reaction. An example of these proteins are those containing heme groups, where the Fe ion in the porphyrin ring changes valence state depending on the whether an electron is accepted or given up by the protein.

The metalloporphyrin group consists of an organic part and a central heavy atom. If the metal is iron, it is called the heme group. The structure of the porphyrin ring, shown in Figure 1.4, consists of four pyrrole rings linked by methene bridges to form a tetrapyrrole ring. The iron atom binds covalently to the four nitrogens in the center of the porphyrin. The iron can form two additional bonds, one on either side of the heme plane.

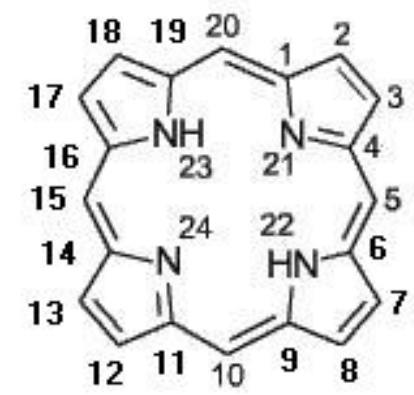

Figure 1.4 The structure of a porphyrin ring.

The $2,3,7,8,12,13,17$ and 18 positions are commonly referred to as " $\beta$ positions" (i.e. of the pyrrole rings). Similarly, positions at 1, 4, 6, 9, 11, 14, 16 and 19 are referred to as " $\alpha$-positions," while those at 5, 10, 15 and 20 are referred to generically as "meso-positions."

There are many types of heme proteins, such as myoglobin, hemoglobin, cytochrome P450, etc. The main function of myoglobin $(\mathrm{Mb})$ is oxygen storage, but it also transports oxygen in the cell and may also carry energy. The detailed structure of the $\mathrm{Mb}$ protein is showed in Fig. 1.5A. The heme group is embedded in the folded 
polypeptide chain. If the iron ion in the heme group is in the +2 oxidation state, it is called the ferrous form. In this case, the heme binds $\mathrm{O}_{2}$ (Fig. 1.5B), and myoglobin becomes oxymyoglobin. If the iron ion is in the +3 ferric form, myoglobin becomes metmyoglobin and the heme will not bind $\mathrm{O}_{2}$.
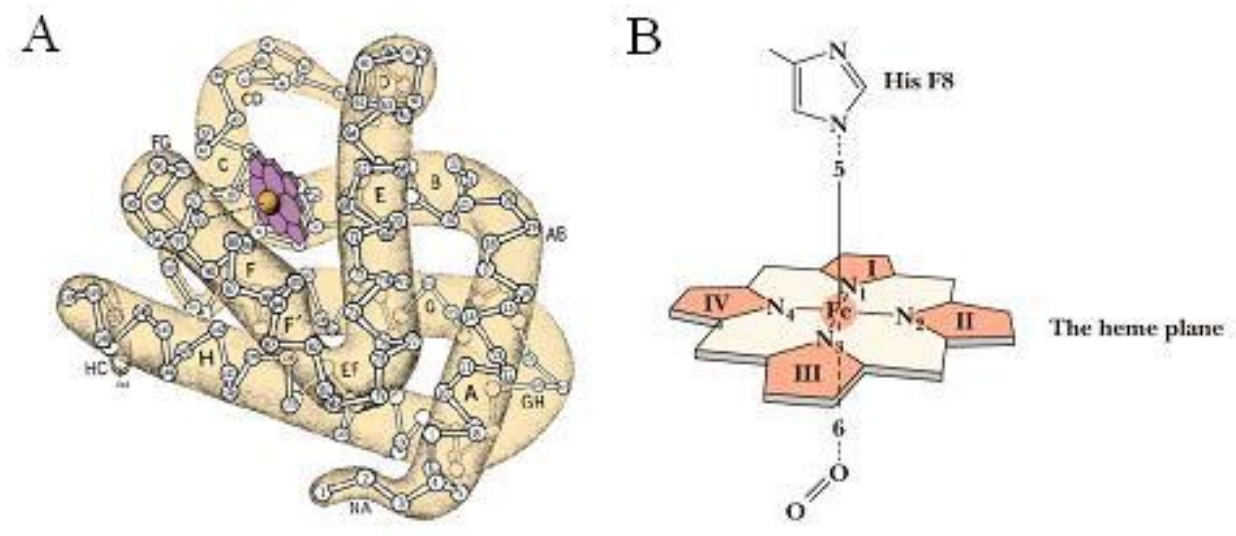

Figure 1.5 A: Detailed structure of the myoglobin protein. B: The heme group in oxymyoglobin, a porphyrin ring system complex with an iron ion. (from Ref. 5).

Figure $1.5 \mathrm{~B}$ shows the heme group in oxymyoglobin. There are six ligands around an iron ion. Four ligands share a common plane, the heme plane, which is composed by the nitrogen atoms of the four pyrrols. The fifth and sixth ligands lie above and below the heme plane. In myoglobin, the fifth ligand is His F8. In oxymyoglobin, the sixth ligand is $\mathrm{O}_{2}$ and in deoxymyoglobin, the sixth ligand is vacant. In metmyoglobin, for the ferric atom, the sixth ligand is occupied by a $\mathrm{H}_{2} \mathrm{O}$ molecule-filled $\mathrm{O}_{2}$ site. The iron in the active $\mathrm{Mb}$ is in the ferrous state and is formally written as $\mathrm{Fe}^{2+}$.

\subsubsection{Redox Reaction}

A redox reaction (shorthand for reduction/oxidation reaction) describes all chemical reactions in which the oxidation number (oxidation state) of an atom is changed. The term redox comes from the two concepts of reduction and oxidation. Oxidation describes the loss of electrons by a molecule, atom or ion. Reduction describes the gain of electrons by a molecule, atom or ion. The iron Fe ion in the porphyrin ring of a heme- 
containing protein changes valence state depending on the whether an electron is accepted or given up by the protein.

\subsubsection{Crystal Field Theory (CFT)}

Crystal field theory describes the electronic structure of transition metal compounds which can be considered coordination complexes. Figure 1.6 shows the splitting of $d$ orbitals according to CFT. In the free transition metal ion, the five $d$ orbitals are degenerate. In the proximity of a negative spherical charge distribution, the energy of the orbitals of the transition metal ion increase due to the Coulomb repulsion between the electrons in the ion and the spherical charge. ${ }^{6,7}$ In the presence of a nonspherically symmetric field, the degeneracy of the orbitals is removed. For example, in an octahedral field generated by six point charges equidistant from the ion, the wavefunctions of some of the $d$-orbitals will cause a stronger electrostatic interaction with the point charges than others, resulting in a loss of degeneracy.

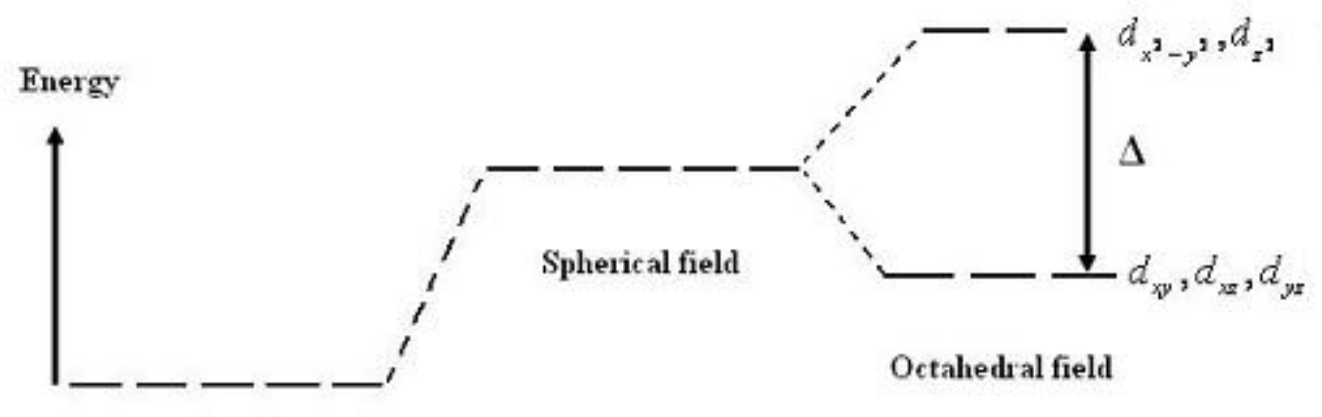

Free ion

Figure 1.6 Splitting of the d orbitals in an octahedral field.

\subsubsection{Ligand Field Theory (LFT)}

CFT describes certain properties of coordination complexes and emphasizes electrostatic interactions between the ligand electrons with the d-electrons on the metal, but it does not describe bonding. Combined with molecular orbital theory, CFT was developed to the more realistic and complex ligand field theory (LFT).

Ligand field theory (LFT) is an application of molecular orbital theory that describes the chemical bonding in transition metal complexes. For the octahedral 
complexes, the transition metal ion has six hybridized atomic orbitals to engage its ligands. The five $\mathrm{d}$ orbitals will lose part of their degeneracy according to crystal field theory. If combined with molecular orbital theory, the five $d$ orbitals may lose their degeneracy. ${ }^{8}$ Figure 1.7 show the calculated orbital structure and relative energy of oxymyoglobin. ${ }^{9}$

The electronic state of $\mathrm{Fe}$ in myoglobin can be elucidated by the LFT. As mentioned above, the $\mathrm{Fe}$ ion in myoglobin has two oxidation states: the ferrous $\left(\mathrm{Fe}^{2+}\right)$ and the ferric $\left(\mathrm{Fe}^{3+}\right)$ states. Considering its high spin and low spin states, the Fe ion has four electronic states, shown in Figure 1.8.

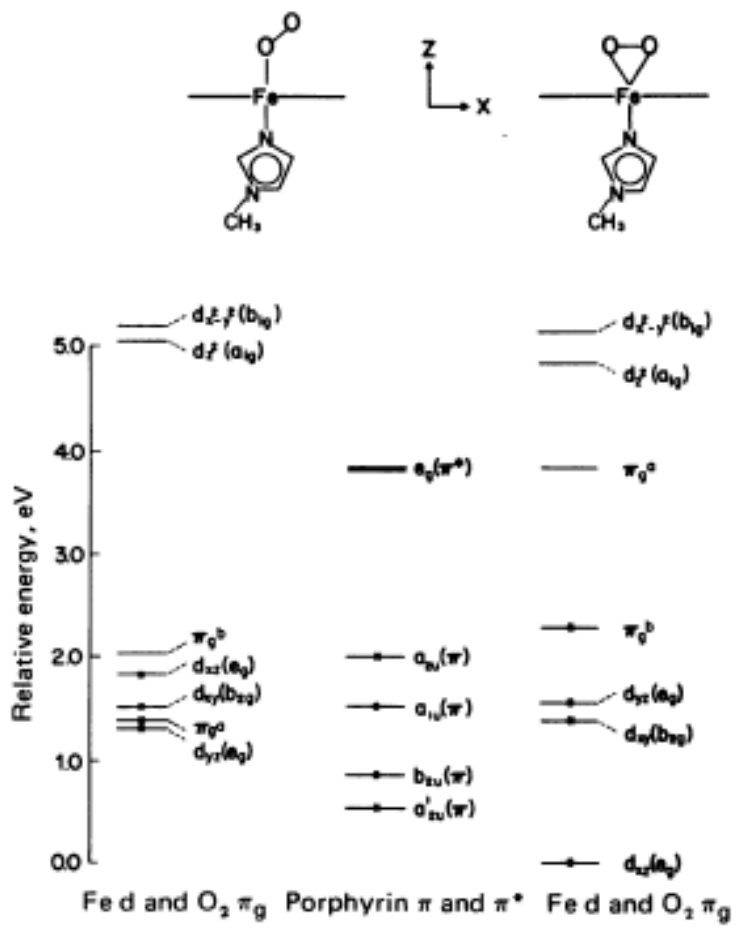

Figure 1.7 Calculated orbital structure and relative energy of oxymyoglobin (from Ref. 9). 


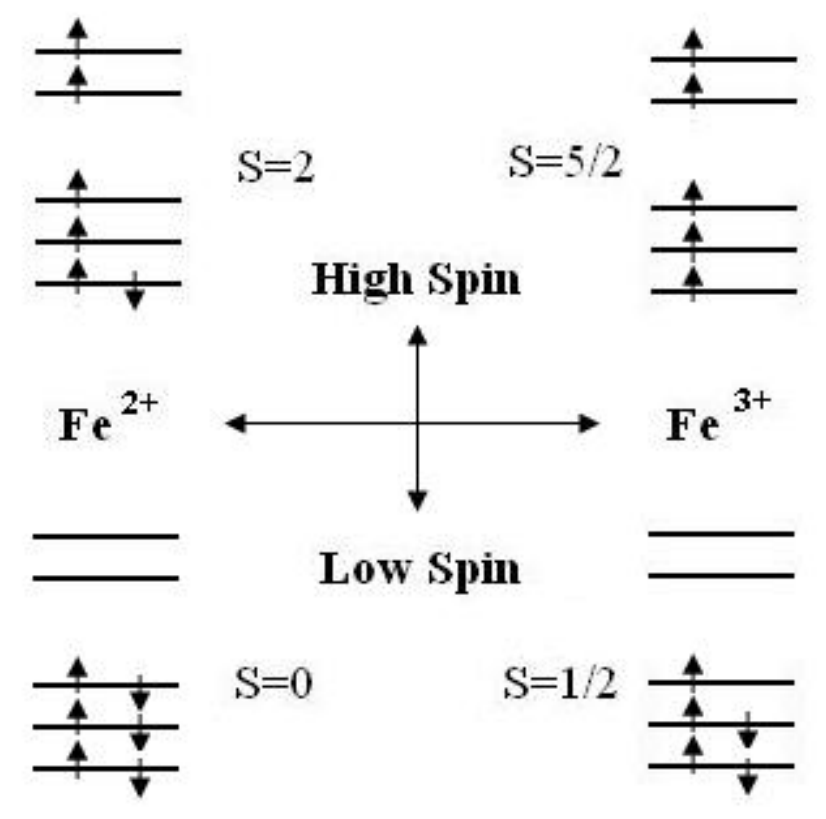

Figure 1.8 The electronic states of iron in myoglobin.

\subsubsection{Protein Denaturation}

The destruction of the structural order of a protein is called denaturation, which is accompanied by loss of function. Proteins can be denatured by changing the $\mathrm{pH}$, heating or exposing them to organic solvents. Anything that breaks the bonds and electrostatic attractions responsible for maintaining the structure of protein will cause the protein to denature.

For most biological proteins, their biological function will be lost when denatured. For example, enzymes lose their activity, because the substrates can no longer bind to the active site. For some proteins, denaturation is reversible, that is, the proteins will re-form their structure and can regain their native state when the denaturing conditions are removed.

\subsection{Electron Transfer (ET)}

Electron transfer (ET) is a fundamental process by which an electron moves from one atom or molecule to another atom or molecule. ET underlies many physical, chemical and biological reactions. There are many examples of ET in organic molecules with metal complexes. Electron transfer from a molecule (the donor) with high-energy to 
a molecule (the acceptor) with a lower-energy can be separated into a series of intermediate redox reactions called an electron transport chain. ${ }^{10}$ Electron transfer reactions where an electron is transferred from an donor D to an acceptor A can be written as

$$
D+A \rightarrow D^{+}+A^{-}
$$

If the electron is transferred through a molecular bridge (B), which is either connected to a donor and acceptor molecular units, or to two metal electrodes, this reaction can be described as follows

$$
D B A \rightarrow D^{+} B A^{-}
$$

There are three kinds of ET reactions. (1) Inner sphere electron transfer. In this case, the two redox centers are covalently linked during the ET. The reactant and oxidant share a ligand in their inner or primary coordination spheres with the electron being transferred across a bridging group. (2) Outer sphere electron transfer. In this case, the participating redox centers are not linked via any bridge during the ET event. Instead, the electron "hops" from the reducing center to the acceptor. Outer sphere electron transfer can occur between differing chemical species or between identical chemical species that differ only in their oxidation state. (3) Heterogeneous electron transfer. In this case, an electron is moved between a chemical species and a solid-state electrode.

\subsection{Marcus Theory}

The generally accepted electron transfer theory was developed by Rudolph A. Marcus to explain outer sphere electron transfer. The Marcus theory is based on a transition-state approach. The outer sphere electron transfer is the transfer of electrons between two metal spheres enabled by introducing reorganization energy and electronic coupling to the reaction coordinate diagram typically portraying the Gibbs' Free Energy and thermodynamic transition state. The Marcus theory was later extended to inner sphere electron transfer and heterogeneous electron transfer.

In the view of Marcus theory, all the nuclear motions of the reactant and the surrounding environment are regarded approximately as a single, simple harmonic oscillator potential along a reaction coordinate. ${ }^{2}$ In Figure 1.9, the left parabola 
represents the potential energy surface for the nuclear motion of the reactants in the initial state (where the electron is still on the donor molecule or group), and the right parabola represents the potential energy surface for the nuclear motion of the products in the final state (after the electron has transferred from the donor to the acceptor).

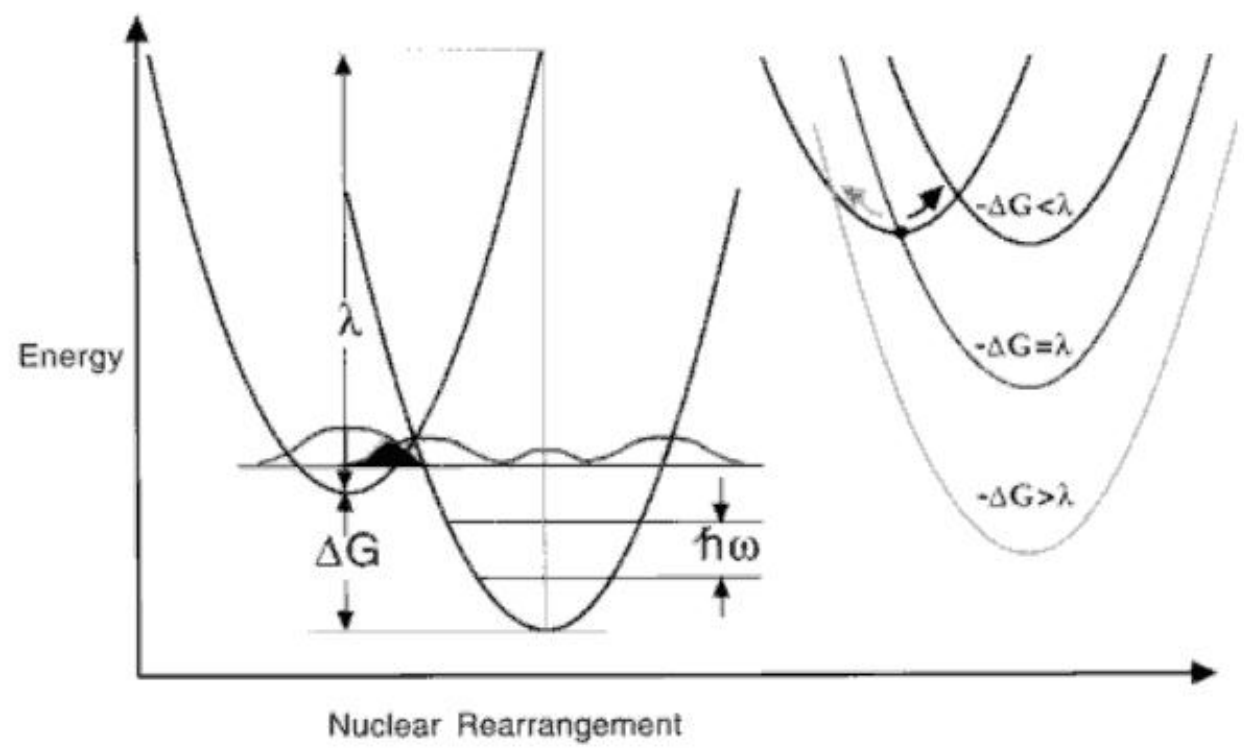

Figure 1.9 Energy diagram of the Marcus theory (from Ref. 2).

There are two fundamental points of the theory: 1 . The reorganization energy required to optically excite an electron from an electron donor to an electron acceptor is intimately related to the activation energy for thermal electron transfer process. 2 . The rate of electron transfer from one redox site to another will increase with the thermodynamic driving force for the reaction to a point. When the driving force becomes too large the electron transfer rate will decrease.

The basic equation of the Marcus theory is

$$
k_{e t}=\frac{2 \pi}{\hbar} H^{2}{ }_{A B} \frac{1}{\sqrt{4 \pi \lambda k_{B} T}} \exp \left(\frac{-(\lambda+\Delta G)^{2}}{4 \lambda k_{B} T}\right),
$$

where $k_{e t}$ is the rate of electron transfer, $H_{A B}$ is the electronic coupling between the initial and final states, $\lambda$ is the reorganization energy, $\Delta G$ is the total Gibbs free energy 
change for the electron transfer reaction, $k_{B}$ is the Boltzmann constant, and $T$ is the temperature. According to the semiclassical Marcus' theory, an electron transfer takes place when a thermal fluctuation of the solvent, or that of the nuclear coordinate of the donor and acceptor, shifts the donor and acceptor electronic states into resonance.

\subsection{Redox Potential}

The oxidation-reduction reactions of proteins containing heme groups can occur at the right conditions. The ability of an organism to carry out oxidation-reduction reactions depends on the oxidation-reduction state of the environment, or its reduction potential.

The redox potential (also known as oxidation / reduction potential) describes the affinity of a chemical species for electrons. The more negative the redox potential, the less the species' affinity for electrons and the tendency is toward oxidation. Usually, the redox potential is measured in volts $(\mathrm{V})$ or millivolts $(\mathrm{mV})$. Because the absolute potentials are difficult to measure accurately, the reduction potential of a chemical species is defined relative to the standard hydrogen electrode (which is set at 0.00 volts). Any system or environment that accepts electrons from a normal hydrogen electrode is a half cell that is defined as having a positive redox potential; any system donating electrons to the hydrogen electrode is defined as having a negative redox potential.

\subsection{Cyclic Voltammetry (CV)}

Cyclic voltammetry (CV) can be used to measure the redox potential of proteins. $\mathrm{CV}$ is one of the most frequently used electrochemical methods because of its relative simplicity and its high information content. There are three electrodes used in this technique: the working electrode, the reference electrode, and the counter electrode. In this experiment, the working electrode potential is ramped linearly versus time like linear sweep voltammetry. Cyclic voltammetry takes the experiment a step farther then linear sweep voltammetry which ends when it reaches a set potential. When cyclic voltammetry reaches a set potential the working electrode's potential ramp is inverted. This inversion can happen multiple times during a single experiment. The current flowing at the working electrode is plotted versus the applied voltage to give the cyclic 
voltammogram trace. Cyclic voltammetry is generally used to study the electrochemical properties of analyte in solution. ${ }^{11,12}$ There are two important examples of this type of measurement:

\section{Fe(III)-protoporphyrin IX}

Fe(III)-protoporphyrin IX [FePP, Fig. 1.10(C)] was studied in Tao's STM experiment ${ }^{3}$ sketched in Fig. 1.10(A). Its CV results were obtained with a sweeping rate of $0.2 \mathrm{~V} / \mathrm{s}$ (Fig. 1.10(D)). The redox potential measured in the $\mathrm{CV}$ was negative which means that the initial state of the $\mathrm{Fe}$ was $3+$ at $V_{G}=0$. Its redox level was above the Fermi level of electrodes (showed in Fig. 1.10(B)) and therefore the electrochemical potential of the molecule had a positive value. The energy diagram of this molecule, shown in Fig. 1.10(B), was developed according to this CV result.
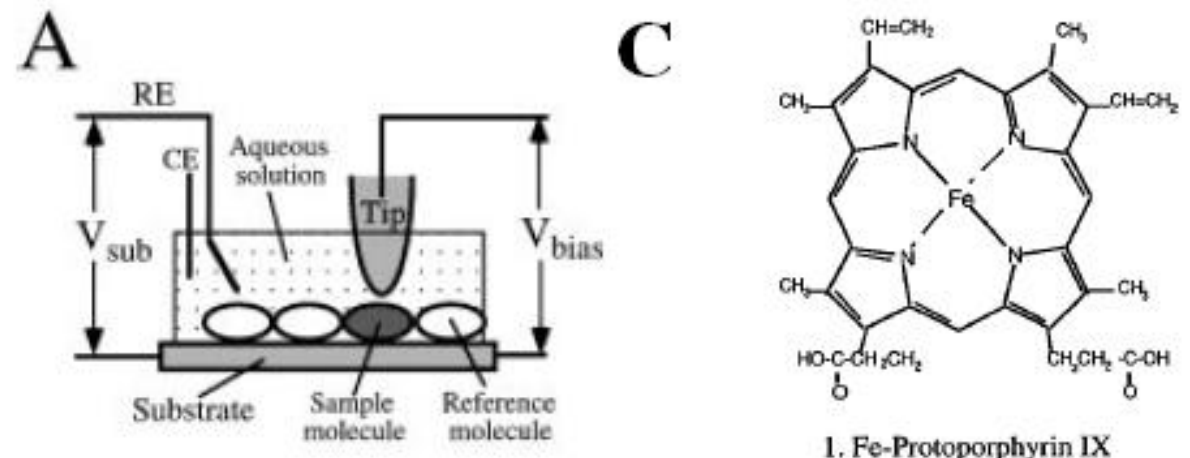

1. Fe-Protoporphyrin IX
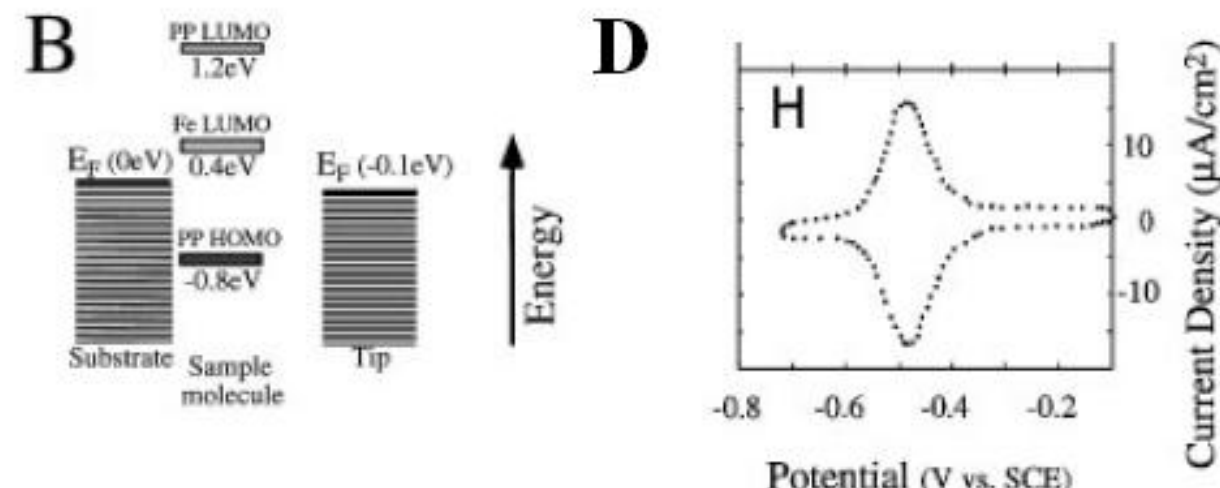

Figure 1.10 Tao's results (from Ref. 3). (A) Schematic of Tao's STM experiment. (B) Energy diagram of the molecule. (C) The structure of the molecule. (D) The CV result of the molecule.

2. $\left.\left[\mathrm{C}_{0}\left(\mathrm{tpy}-\left(\mathrm{CH}_{2}\right)_{5}-\mathrm{SH}\right)\right)_{2}\right]^{2+}$ and $\left[\mathrm{C}_{\mathrm{o}}(\mathrm{tpy}-\mathrm{SH})_{2}\right]^{2+}$

$\left.\left[\mathrm{C}_{0}\left(\text { tpy- }\left(\mathrm{CH}_{2}\right)_{5}-\mathrm{SH}\right)\right)_{2}\right]^{2+}$ (showed in Fig. 1.11(A)) and $\left[\mathrm{C}_{\mathrm{o}}(\mathrm{tpy}-\mathrm{SH})_{2}\right]^{2+}$ were used in Park's experiment. ${ }^{13} \mathrm{CV}$ measurements of $\left[\mathrm{C}_{0}(\text { tpy-SH })_{2}\right]^{2+}$ are shown in Fig. 1.11(B). 
The redox potential measured in the $\mathrm{CV}$ was positive which means that the initial state of the cobalt ion was $2+$ at $V_{G}=0$. Its redox level was below the Fermi level of electrodes. So, the electrochemical potential of the molecule had a negative value. The energy diagram of these molecules is shown in Fig. 1.11(C) based on this CV result.

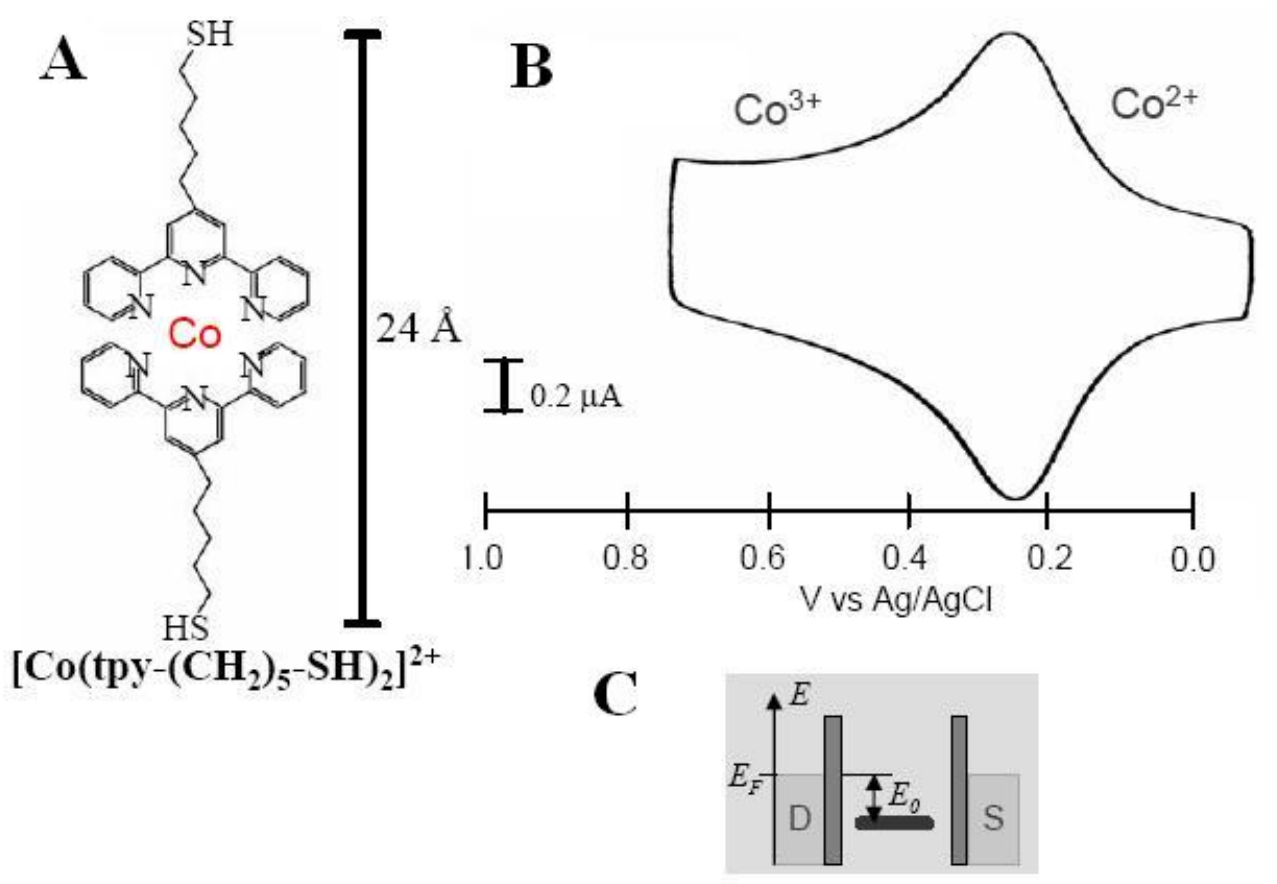

Figure 1.11 Park's results (from Ref. 13). (A) The structure of the molecule. (B) The CV result of the molecule. (C) Energy diagram of the molecule.

\subsection{Electrochemical STM (EC-STM)}

Single-molecule electronic devices were first proposed by Aviram and Ratner in 1974. ${ }^{14}$ Since then, researchers have developed various experimental devices to measure the electrical conductance of molecules. However, the study of single molecules was not practical until the invention of scanning tunneling microscopy (STM). The first experiment with a single molecule with redox reactions was reported by Tao based on STM measurements. ${ }^{3}$ Figure 1.12 shows an electrochemical scanning tunneling microscope (EC-STM). ${ }^{15}$ This device can be regarded as the combination of conventional STM and cyclic voltammetry. It can work in liquid on a conductive and atomically flat substrate. The role of the conductive substrate is that of the working electrode. The tip can be considered as another working electrode. The bipotentiostat controls the potential of the substrate and the tip. In EC-STM, the electrochemical gate 
(the substrate) is used to shift and align the molecule's redox level to the metal contacts' Fermi level, and the bias voltage between the tip and the substrate is kept constant with respect to the reference electrode when changing the gate voltage.

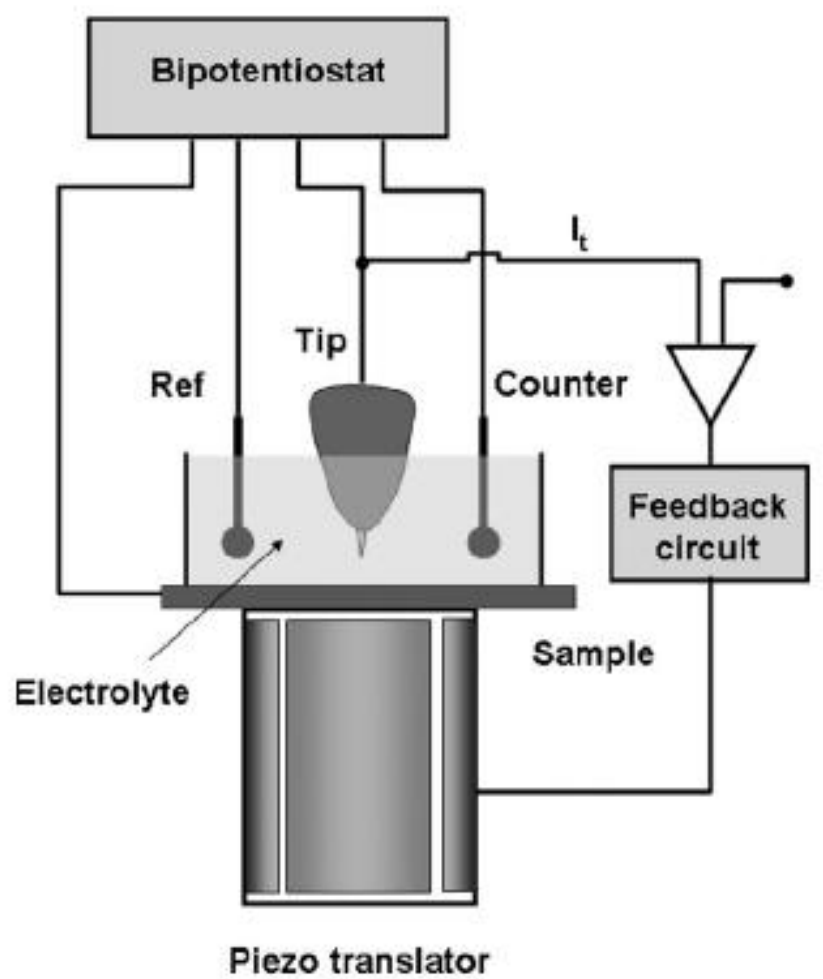

Figure 1.12 EC-STM configuration (from Ref. 15).

\subsection{Some ET Models}

\subsubsection{Two-Step ET Model}

This model has been used to describe how electron transfer by redox levels in ECSTM. Figure 1.13 shows energy diagram of a redox molecule in EC-STM. ${ }^{16}$ Electrons tunnel from the negatively biased electrode (substrate) to the positively biased electrode (tip) by resonating with the molecular redox level.

The initial equilibrium redox level position shown in Fig. 1.13 is vacant, which is above the Fermi level of electrodes. This vacant energy level can be shifted close to the Fermi level of electrodes by changing the substrate voltage and the bias voltage. Nuclear configurational fluctuations can also shift the vacant redox level close to the Fermi level of the negatively biased electrode where interfacial ET occurs. ${ }^{16,17}$ In Fig. 1.13, $e \xi \eta$ is 
the effective overpotential and $e V_{\text {bias }}$ is the bias potential energy with respect to the substrate. At a negative bias voltage, first the electron tunnels from the left electrode to the vacant redox level and then from the temporarily occupied redox level to the right electrode by thermal activation. The occupied level will be trapped below the Fermi level of two electrodes by further vibrational relaxation. ${ }^{16}$ This is the two-step process. A corresponding ET sequence would occur in the opposite direction at a positive bias voltage or when the redox level at equilibrium is initially occupied and located below the Fermi level of the positively biased (right) electrode. ${ }^{16,17}$

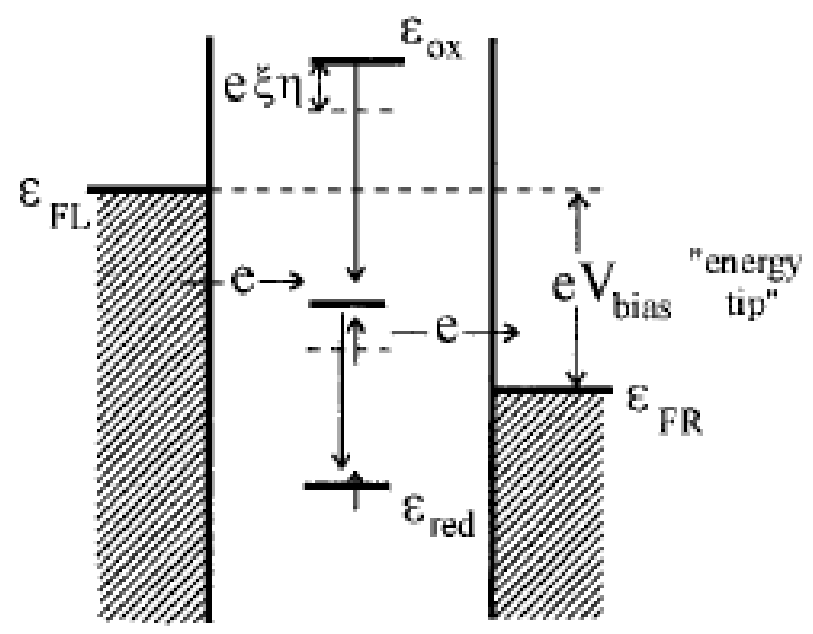

Figure 1.13 Energy diagram of EC-STM of redox molecule (from Ref. 16).

When electron transfer is much slower than typical redox energy fluctuations (typically, when the electronic coupling of the redox centre to the electrodes is small), the overall tip-to-substrate electron transport can be seen as the result of two processes that occur sequentially. First, one ET process occurs from the tip to the redox molecule, then another ET from the molecule to the substrate. ${ }^{15,16}$ Note that the second process has no memory of where the molecular electrons come from, i.e., there is no correlation between the two processes. ${ }^{15}$ This model predicts that the maximum current is obtained when the redox level of the molecule is mid-way between the Fermi levels of the two electrodes. Figure 1.14 show the tunneling current vs. substrate potential for EC-STM. ${ }^{18}$ Figure 1.14A shows that the redox level is empty and located above the Fermi levels of the two electrodes. Figure 1.14B shows that an amplifying current channel opens when the redox 
level is between the Fermi levels of the two electrodes. Figure 1.14C shows that the redox level occupation leads to level trapping below the Fermi levels of two electrodes.

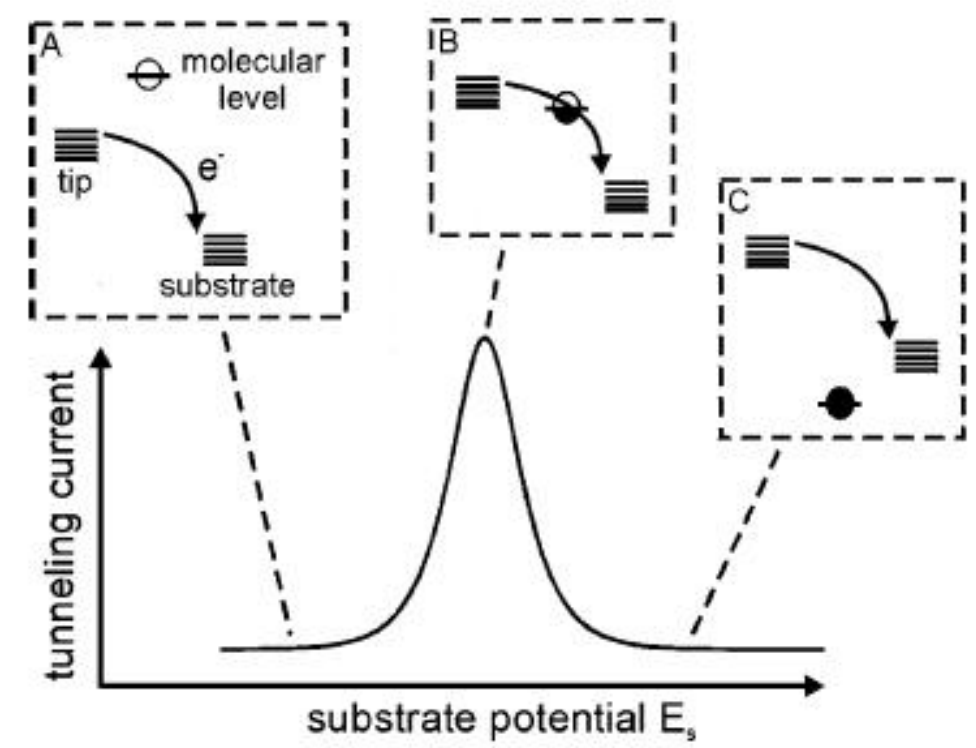

Figure 1.14 Tunneling current vs. substrate potential in EC-STM (from Ref. 18).

\subsubsection{Resonant Tunneling Model}

This model is only effective when the starting occupied tip electron level, the intermediate molecular level and the final empty substrate level all have the same energy. ${ }^{15}$ The mechanism predicts that the current reaches a peak when the empty state is shifted to the Fermi levels of the two electrodes by the gate potential. This implies that the ET between the tip and the substrate is much faster than the typical timescale of the fluctuations of the redox level energy (fluctuations are slow with respect to electron tunneling). Figure 1.15 shows the energy diagram of the resonant tunneling model. 


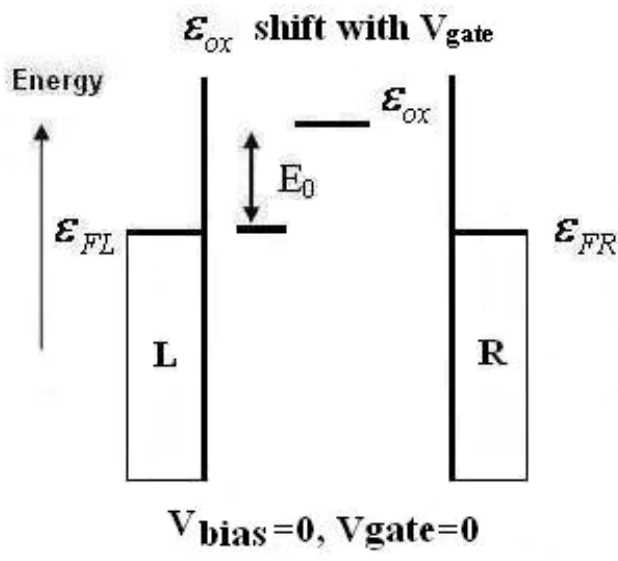

(a)

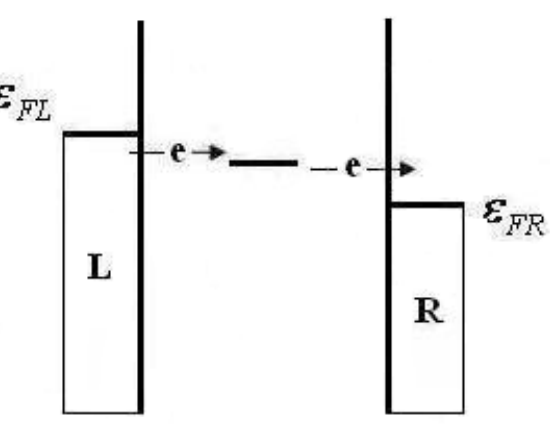

(b)

Figure 1.15 Energy diagram of the resonant tunneling model. (a) The redox level is above the Fermi levels of two electrodes. (b) The redox level is shifted to the Fermi levels of two electrodes by the gate potential.

\subsection{Summary}

In this chapter, the protein structure and some basic concepts that necessary for the description of the Marcus theory was reviewed. The concepts of redox potential and cyclic voltammetry were introduced. Finally, EC-STM techniques and some models for electron transfer by redox centers were discussed. Electron transfer through individual proteins has been probed using EC-STM in aqueous solution at room temperature. Considering the limitations of the poor energy resolution at room temperature and lacking one independent gate electrode for the EC-STM technique, it is necessary to probe the heme proteins with ordinary transistor geometry at cryogenic temperature in order to provide more detailed information for the electron transfer mechanism by the protein. 


\section{Chapter 2}

\section{Basic Theories}

\subsection{Overview}

The mechanism of electron transfer through an insulating medium relies on quantum mechanical electron tunneling. The importance of electron tunneling in biology was clearly shown in early experiments. ${ }^{19,20}$ In the first section of this Chapter, some basic tunneling phenomena will be explained, including inelastic electron tunneling, elastic electron tunneling and resonant electron tunneling.

The single electron transistor (SET) theory, which is also known as the Coulomb Blockade theory, has been introduced in a number of review articles and theses. ${ }^{13,21-23}$ To date, single electron transport behavior has been observed in devices made from single molecules with redox states. They include devices based on $\left[\mathrm{Co}\left(\operatorname{tpy}-\left(\mathrm{CH}_{2}\right)_{5^{-}}\right.\right.$ $\left.\mathrm{SH})_{2}\right]^{2+}$, OPV5, and some molecules with one Co ion inside. ${ }^{24-26}$ In these systems, it is possible to treat the behavior of the single molecule as that of a quantum dot. By applying a bias voltage between the electrodes, as well as a gate voltage to the sample, it is possible to probe the molecular energy levels and observe characteristic single-electron transistor (SET) behavior, such as a Coulomb blockade or a Kondo resonance under the right conditions. ${ }^{24-26}$ In the next section of this chapter, SET theory for the single-level molecule will be discussed. Finally, the Kondo effect will be reviewed simply.

\subsection{Inelastic Electron Tunneling}

Vibrational spectroscopy with tunneling electrons was first observed by Jaklevic and Lambe in 1966 when they studied the resonant tunneling from molecules adsorbed in buried metal-oxide-metal junctions due to localized molecular vibrational modes. ${ }^{27,28}$ This experiment showed that electrons tunneling through the $\sim 3 \mathrm{~nm}$ thick oxide were able to excite vibrational modes of the sandwiched molecules. This discovery marked the birth of a new spectroscopic tool which utilizes inelastic electron tunneling processes. Since then, inelastic electron tunneling spectroscopy (IETS) has been developed as a 
powerful spectroscopic tool for chemical identification, bonding investigation, trace substance detection, etc.

Figure 2.1 shows the energy diagram for inelastic tunneling with a vibrational excitation and the corresponding plots $\left(I-V, d I / d V\right.$ and $\left.d^{2} I / d V^{2}\right){ }^{29-31}$ When a negative bias voltage is applied to the left electrode, an electron tunnels from an occupied state on the left side into an empty state on the right side. If energy is conserved (process a in Fig. 2.1(a)), this is an elastic process. During this process the current increases linearly with the applied small bias (showed in Fig. 2.1(b)). If there is a vibrational mode with a frequency of $\omega$ localized inside this barrier, then when the applied bias is large enough such that $e V \geq \hbar \omega$ (the threshold for excitation of a vibrational mode), the electron can lose a quantum of energy of $\hbar \omega$ to excite the vibrational mode and tunnel into another empty state (process b in Fig. 2.1(a)). This opens an inelastic tunneling channel for the electron and its overall tunneling probability is increased. Thus the total tunneling current has a kink as a function of the applied bias (showed in Fig. 2.1(b)). This kink becomes a step in the differential conductance $(d I / d V)$ plot, and becomes a peak in the $d^{2} I / d V^{2}$ plot. When the energy of the electron is near the threshold for excitation of a vibrational mode, the electron can directly tunnel elastically or through a vibrational mode.

Since only a small fraction of electrons tunnel inelastically, the conductance step is too small to be conveniently detected. ${ }^{31}$ Usually, a phase sensitive detector ("lock-in") second harmonic detection technique is used to directly measure the peaks of the second derivative of $I(V) .{ }^{32}$ 

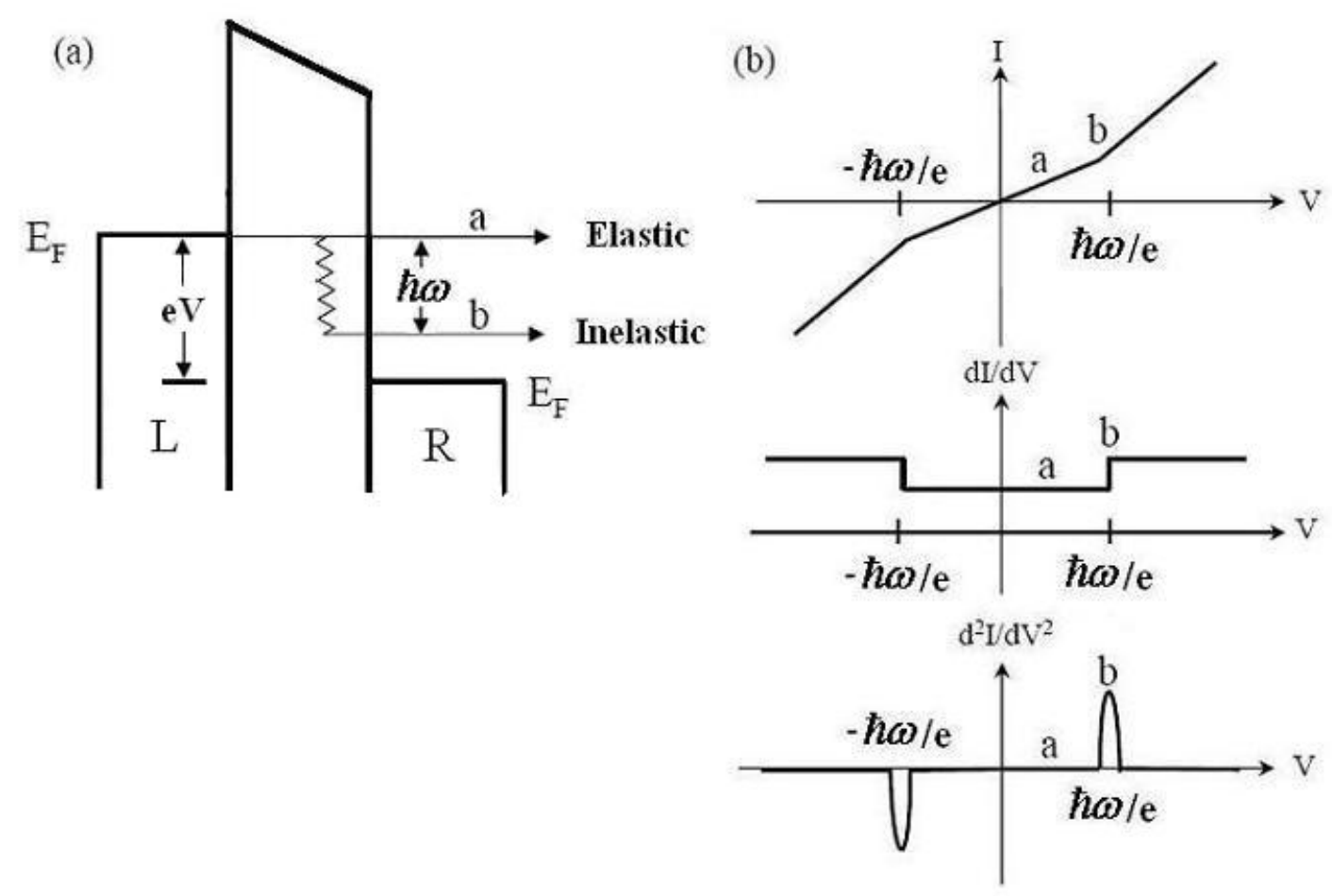

Figure 2.1 Diagram of the elastic and inelastic electron tunneling (revised from Ref. 30).

\subsection{Elastic Electron Tunneling}

\subsubsection{Rectangular Potential Barrier}

Fig. 2.2 shows a rectangular barrier with a barrier height of $V_{0}$ and a barrier width

of $a$. An electron with energy $E<V_{0}$ incoming from the left would be totally reflected by the barrier according to classical physics. However from the quantum mechanical point of view, due to the wave nature of electron, there is some probability that the electron will tunnel through this barrier to the other side. Schrödinger's equation can be used in different ranges to get the transmission coefficient of this potential. 


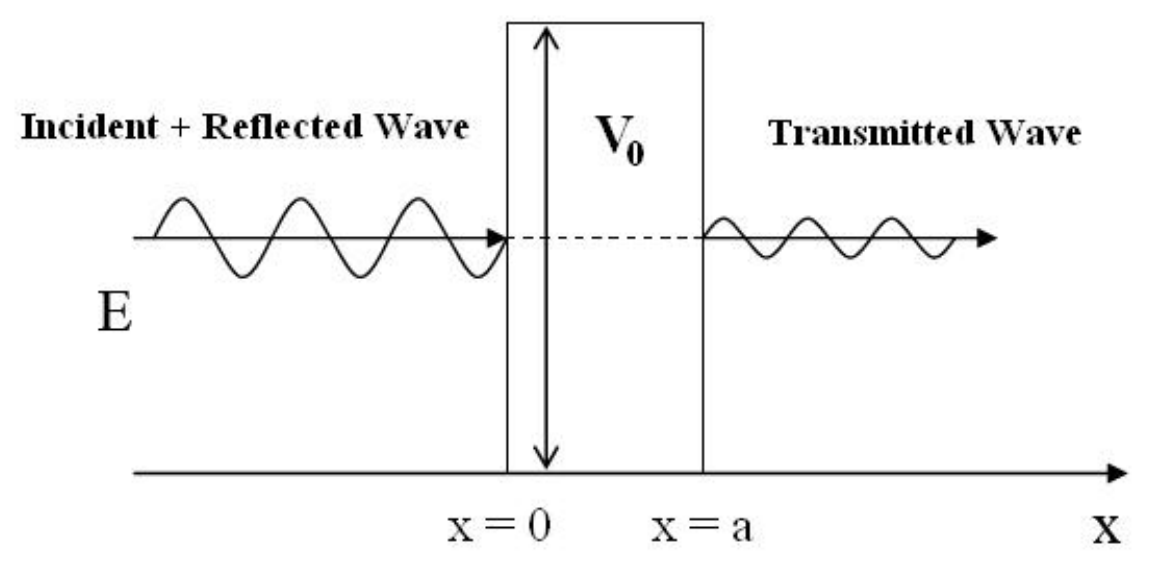

Figure 2.2 An electron with energy $E$ tunnels through a rectangular barrier.

Schrödinger's equation for an electron outside the barrier $(x<0, x>0)$ is

$$
\frac{d^{2}}{d x^{2}} \psi+\frac{2 m E}{\hbar^{2}} \psi=0
$$

And the solutions in there two regions can be written as

$$
\begin{array}{ll}
\psi_{1}(x)=e^{i k_{1} x}+\operatorname{Re}^{-i k_{1} x}, & x<0 \\
\psi_{3}(x)=S e^{i k_{1} x}, & x>0
\end{array}
$$

where $k_{1}=\sqrt{2 m E / \hbar^{2}}$, and only the transmitted wave is considered in the $x>$ a region.

The Schrödinger's equation for an electron inside the barrier $(0<x<a)$ is

$$
\frac{d^{2}}{d x^{2}} \psi-\frac{2 m\left(V_{0}-E\right)}{\hbar^{2}} \psi=0
$$

And the solutions in this region can be written as

$$
\psi_{2}(x)=A e^{k_{2} x}+B e^{-k_{2} x}
$$

where

$$
k_{2}=\sqrt{\frac{2 m}{\hbar^{2}}\left(V_{0}-E\right)} .
$$

The values of $\mathrm{R}$ and $\mathrm{S}$ can be obtained by using the wave functions $\psi$ and their first derivatives at $x=0$ and $x=a$, and thus the reflection and transmission coefficients are

$$
|R|^{2}=\frac{\left(k_{1}^{2}+k_{2}{ }^{2}\right)^{2} \sinh ^{2} k_{2} a}{\left(k_{1}{ }^{2}+k_{2}{ }^{2}\right)^{2} \sinh ^{2} k_{2} a+4 k_{1}{ }^{2} k_{2}{ }^{2}} ; T=|S|^{2}=\frac{4 k_{1}^{2} k_{2}{ }^{2}}{\left(k_{1}{ }^{2}+k_{2}{ }^{2}\right)^{2} \sinh ^{2} k_{2} a+4 k_{1}{ }^{2} k_{2}{ }^{2}},
$$


where $|R|^{2}+|S|^{2}=1$.

Now, we discuss the transmission coefficient $T$ :

(1) $T \neq 0$ indicates that the electron can tunnel through a potential barrier that is higher than its kinetic energy.

(2)If $k_{2} a>>1$, then $\sinh k_{2} a \approx \frac{1}{2} e^{k_{2} a}>>1$. In this case, the transmission coefficient can be approximated as

$$
T \approx e^{-2 k_{2} a}=\exp \left[-\frac{2 a}{\hbar} \sqrt{2 m\left(V_{0}-E\right)}\right],
$$

which show that $T$ decays exponentially with the barrier width.

(3) For the case where $V_{0}>E, k_{2}>0$ and $\sinh ^{2} k_{2} a>0$, the transmission coefficient is $T<1$, which means no resonant tunneling $(T=1)$ occurs.

In the one dimensional system, the quantum mechanical current density is

$$
J=\frac{-i \hbar}{2 m}\left(\psi * \frac{\partial}{\partial x} \psi-\psi \frac{\partial}{\partial x} \psi^{*}\right) .
$$

The current density of the transmitted wave $\left(\psi_{3}(x)=S e^{i k_{1} x}\right)$ is

$$
j=e \frac{\hbar k_{1}}{m} T \propto T .
$$

\subsubsection{The Square Potential Well}

Fig. 2.3 shows a square potential well of $-V_{0}$ and with a width of $2 a$. An electron with energy $E$ is incoming from the left. According to classical physics, no electrons would ever be turned back. However from the quantum mechanical point of view, the electron will be reflected from the sharp edges at $x=a$ and $x=-a$. 


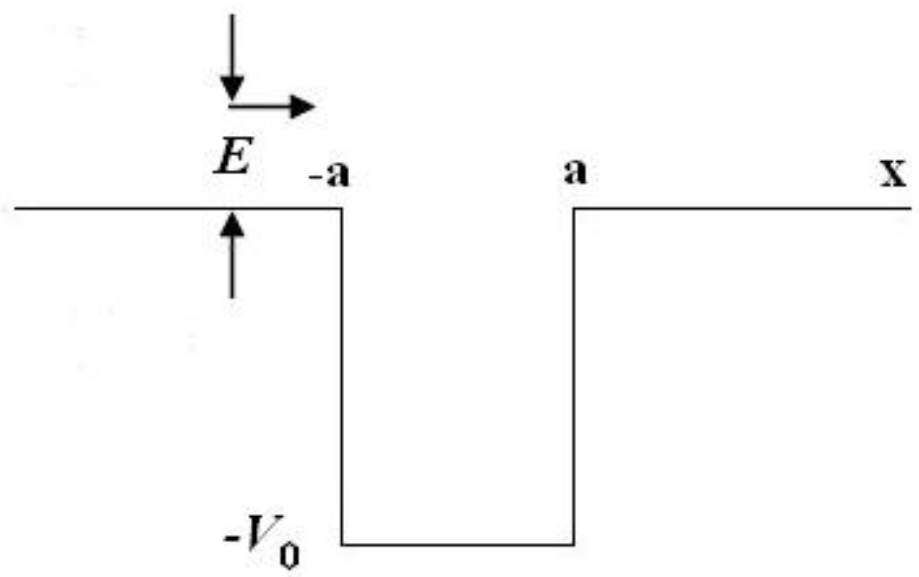

Figure 2.3 An electron with energy $E$ tunnels through a potential well.

The Schrödinger's equation for an electron in the region $x<-a$ is

$$
\frac{d^{2}}{d x^{2}} \psi+\frac{2 m E}{\hbar^{2}} \psi=0 \text {. }
$$

The solution in this region can be written as

$$
\psi_{1}(x)=A e^{i k_{1} x}+B e^{-i k_{1} x},
$$

where $k_{1}=\sqrt{2 m E / \hbar^{2}}$.

Schrödinger's equation for an electron inside the barrier $(-a<x<a)$ is

$$
\frac{d^{2}}{d x^{2}} \psi+\frac{2 m\left(E+V_{0}\right)}{\hbar^{2}} \psi=0 .
$$

The solution in this region can be written as

$$
\psi_{2}(x)=C e^{i k_{2} x}+D e^{-i k_{2} x}
$$

where $k_{2}=\sqrt{\frac{2 m}{\hbar^{2}}\left(E+V_{0}\right)}$.

The solution in the region $x>a$ can be written as

$$
\psi_{3}(x)=E e^{i k_{1} x},
$$

where $k_{1}=\sqrt{2 m E / \hbar^{2}}$.

The values of $A, B, C, D$ and $E$ can be obtained by using the wave functions $\psi$ and requiring that their first derivatives be continuous at $x=-a$ and $x=a$. The transmission coefficient is found to be 


$$
T=\frac{|E|^{2}}{|A|^{2}}=\frac{1}{1+\frac{1}{4}\left(\frac{k_{1}}{k_{2}}-\frac{k_{2}}{k_{1}}\right)^{2} \sin ^{2}\left(2 k_{2} a\right)} .
$$

There are two important cases:

(1)If $k_{1}=k_{2}, T=1$. In this case, $V_{0}=0$, there is no potential well at all, so $T=1$.

(2)If $k_{1} \neq k_{2}$, i.e. $V_{0} \neq 0$, the transmission coefficient $T<1$, indicating that some reflection has taken place. This reflection from an attractive potential is a result of the wave nature of matter.

\subsection{Resonance Tunneling}

\subsubsection{Square Potential Well}

From the equation above, the transmission coefficient $T=1$ when $\sin ^{2}\left(2 k_{2} a\right)=0$, or $k_{2}=\frac{n \pi}{2 a}$, where $n$ is an integer. In this case, the potential well is totally transparent for electron transmission. This phenomenon is called resonance tunneling.

From $k_{2}=\frac{n \pi}{2 a}=\sqrt{\frac{2 m}{\hbar^{2}}\left(E+V_{0}\right)}$, the resonance energy is

$$
E=-V_{0}+\frac{\pi^{2} \hbar^{2}}{8 m a^{2}} n^{2}, \mathrm{n}=1,2,3, \ldots
$$

\subsubsection{One-dimensional Double Barrier}

As explained above, in quantum tunneling through a single barrier the transmission coefficient, or the tunneling probability, is always less than one for an incoming particle with kinetic energy less than the potential barrier height. Consider the potential profile which contains two barriers (located close to each other) in Figure 2.4(a). The transmission coefficient (as a function of the incoming particle energy $E$ ) can be calculated using any of the standard methods. ${ }^{33}$ Resonance tunneling occurs when the energy $E$ of the incident electron is close to certain energies. It is interesting that while the transmission coefficient of a single potential barrier is always lower than one (and decreases with increasing barrier height and width), two barriers in a row can be completely transparent for certain energies of the incident particle. 

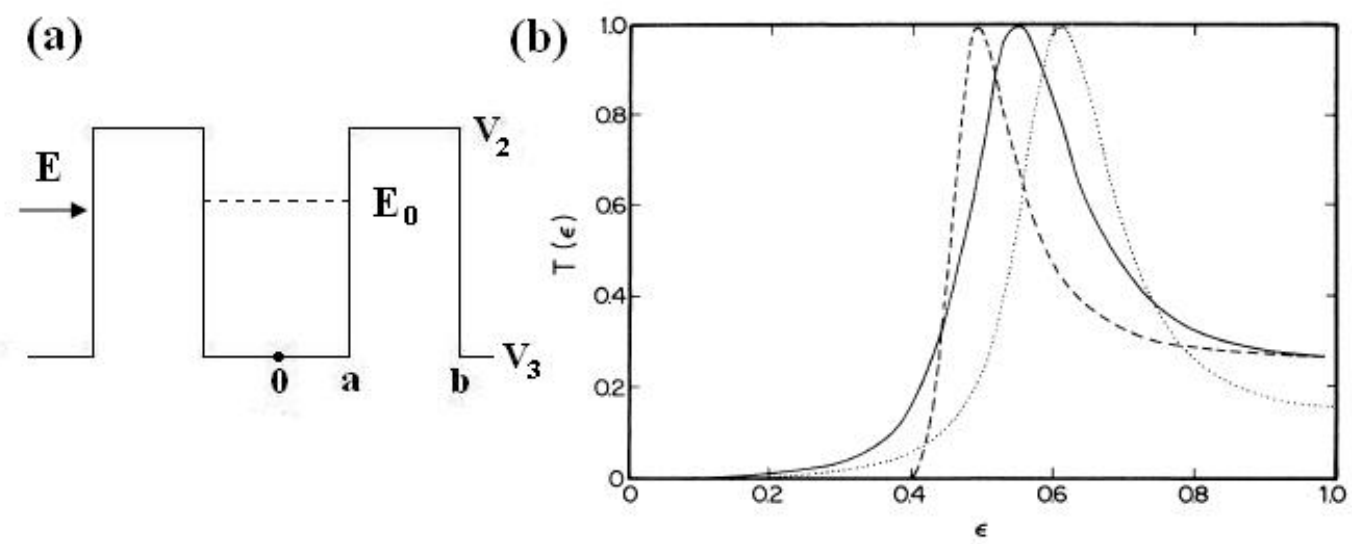

Figure 2.4 (a) Double barriers surrounding a well (b) Its transmission coefficient $T(\varepsilon)$ vs. $\varepsilon=E / V_{2}$ for $V_{3}<E_{0}$ (the resonance energy). The curves shown by dotted, solid and dashed lines are for $V_{3} / V_{2}=-2,0$ and 0.4 , respectively (from Ref. 33).

Assuming that the two barriers are symmetric about the origin and narrow (small $d=b-a)$, the transmission coefficient is

$$
T=\frac{1}{\mu^{2}+\lambda^{2}}=\frac{1}{1+\gamma^{2}},
$$

where $\gamma(E)=\frac{1}{\alpha} A(E)+\alpha B(E)$ with

$A(E)=\left[\sinh \left(K_{2} d\right) \cos (a \sqrt{E})+\beta^{-1} \sin (a \sqrt{E}) \cosh \left(K_{2} d\right)\right]\left[\beta \sinh \left(K_{2} d\right) \sin (a \sqrt{E})-\cosh \left(K_{2} d\right) \cos (a \sqrt{E})\right]$, $B(E)=\left[\sinh \left(K_{2} d\right) \sin (a \sqrt{E})+\beta^{-1} \cosh \left(K_{2} d\right) \cosh (a \sqrt{E})\right]\left[\beta \cosh \left(K_{2} d\right) \sin (a \sqrt{E})-\sinh \left(K_{2} d\right) \cos (a \sqrt{E})\right]$,

and

$$
\alpha \equiv \frac{K_{2}}{K_{3}}=\left(\frac{V_{2}-E}{E-V_{3}}\right)^{1 / 2} ; \beta \equiv \frac{\sqrt{E}}{K_{2}}=\left(\frac{E}{V_{2}-E}\right)^{1 / 2} ; K_{2}=\sqrt{\frac{2 m}{\hbar^{2}}\left(V_{2}-E\right)} ; K_{3}=\sqrt{\frac{2 m}{\hbar^{2}}\left(E-V_{3}\right)} .
$$

Figure 2.4(b) shows the transmission coefficient $T(\varepsilon)$ as a function of the dimensionless energy $\varepsilon=E / V_{2}$ for $V_{3}<E_{0}$ (the resonance energy). The resonant 
condition of the three curves (dotted, sold, and dashed lines) is $V_{3} / V_{2}=-2,0$, and 0.4 respectively, and $a \sqrt{V_{2}}=1, d \sqrt{V_{2}}=1.5$.

Now consider a potential well surrounded by barriers as shown in Figure 2.5. ${ }^{34,35}$ It is assumed that $V(\mathrm{x})$ is symmetric about the origin and the origin is located at the center of the well for simplicity. The turning points are assumed to be at $x=-a,-b, b$ and $a$. The electron is assumed to be incident from the left and its energy $E$ below the peaks of the barriers.

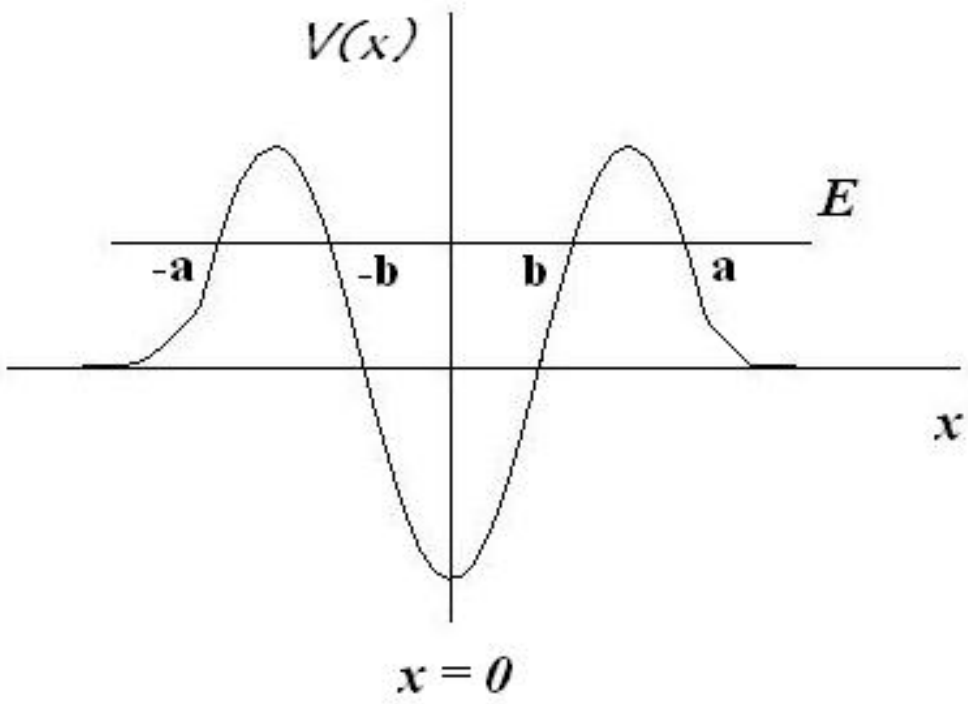

Figure 2.5 A well surrounding two potential barriers.

For this kind of potential, the WKB (Wentzel-Kramers-Brillouin) approximation has been used to describe the wave functions at all regions. The transmission $T$ is

$$
T=\left[1+\frac{1}{4}\left(4 \theta^{2}-\frac{1}{4 \theta^{2}}\right)^{2} \sin ^{2} \frac{1}{2}\left(\pi-\frac{J}{\hbar}\right)\right]^{-1},
$$


where $J$ is the action variable, $J=2 \int_{-b}^{b} p_{w} d x$, and $p_{w}$ is the absolute value of the momentum inside the well. The parameter $\theta=\exp \left(\int_{b}^{a} p 1 \frac{d x}{\hbar}\right)$, where $p 1$ is the absolute value of the momentum inside the barriers.

Transmission resonances occur when

$$
\pi-\frac{J}{\hbar}=-2 N \pi \quad \text { or } \quad J_{N}=\left(N+\frac{1}{2}\right) h, N=0,1,2, \ldots
$$

\subsection{Single Electron Transistor Theory}

An important question is whether single-electron transistors using proteins are feasible. The charging energy $E_{C}=e^{2} / C$, where $e$ is the electron's charge and $C$ is the protein's capacitance, can be roughly estimated by the size of protein. For a sphere with a radius $R$, its self-capacitance is $C=4 \pi \varepsilon_{0} R$ and the charging energy is $E_{C}=e^{2} /\left(4 \pi \varepsilon_{0} R\right)$, where $\varepsilon_{0}$ is the permeattivity of free space. For a protein such as myoglobin with $R=4$ $\mathrm{nm}, E_{C}=360 \mathrm{meV}$, which is much larger than the thermal energy $k_{B} T(25.9 \mathrm{meV}$ at 300 $\mathrm{K} ; 6.65 \mathrm{meV}$ at $77 \mathrm{~K} ; 0.52 \mathrm{meV}$ at $6 \mathrm{~K}$ ). Considering the additional capacitance between the molecule and electrodes, however, the total capacitance is much larger than its self capacitance and this will reduce the charging energy greatly. Therefore, protein-based SETs, in principle, could be observed at moderate cryogenic temperatures for heme proteins. Ideally, proteins with localized, well-defined energy levels are necessary for resonant tunneling to occur.

A few basic concepts will now be introduced following the discussion of other dissertations. $^{13,23}$ In order to treat the problem of a complicated single protein like myoglobin, we assume that resonant electron transfer occurs only through the orbitals of the heme group.

\subsubsection{Basic Concepts of Single Electron Transistor}




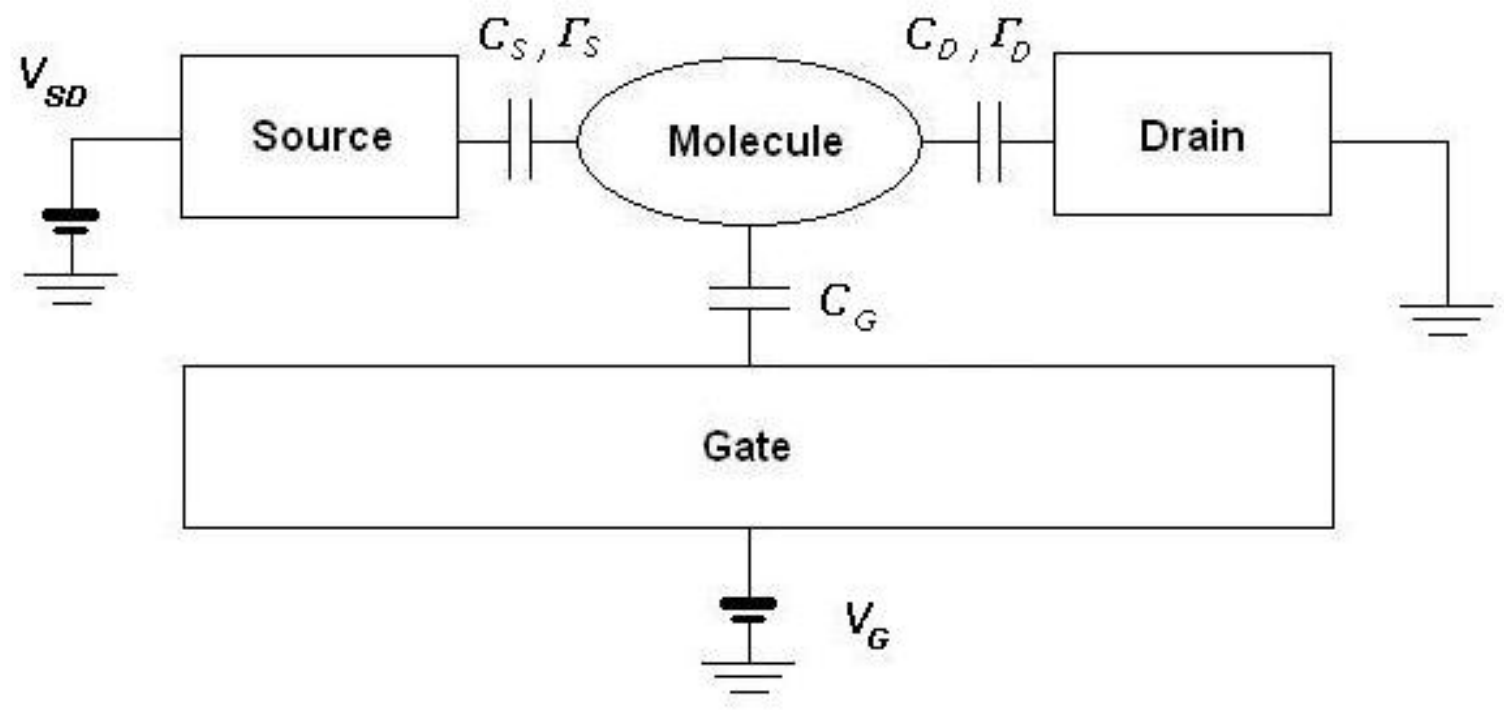

Figure 2.6 A diagram of SET.

Figure 2.6 is a sketch of a single-electron transistor based on a single molecule. The single molecule is coupled capacitatively to three electrodes - the source, drain and gate electrodes with capacitances $C_{S}, C_{D}$ and $C_{G}$, respectively. A change in any of the electrodes' potentials will modify the electrostatic potential of the molecule. The molecule is coupled by an effective tunnel junction to the source and drain electrodes. Therefore, electric current can flow between the source and the drain by electrons tunneling on and off the molecule. The gate electrode is used to shift the molecule's energy levels with respect to the Fermi levels of the source and drain metal electrodes. The rate of electron tunneling between the molecule and the source or drain electrode is represented by the tunneling rates $\Gamma_{S}$ and $\Gamma_{D}$, respectively. $\Gamma_{S}$ and $\Gamma_{D}$ are defined as the number of electrons that tunnel through one of the tunnel barriers per unit time, with units of $s^{-1}$ or $\mathrm{Hz}$.

In this dissertation sequential tunneling as the electron transport mechanism through myoglobin proteins will be used. The sequential tunneling process is described below.

The energy diagram of a single molecule device as illustrated in Figure 2.7. The molecule has $N$ electrons and the solid lines represent all the filled quantized electrochemical levels of the molecule. The lowest dotted line labeled $\mu_{N+1}$ represents an unoccupied molecular energy since it is above the Fermi level of electrodes. In this 
case, the molecule is stable with $N$ electrons and current cannot flow through the molecule. Electric current will flow when an electron can tunnel into the molecule and subsequently off the molecule to the other electrode. When one molecular energy state is available between $\mu_{S}$ and $\mu_{D}$ (the Fermi level of the electrodes), the sequential tunneling process can occur via this state while changing the number of electrons on the molecule between $N$ and $N+1$. An electrical current will flow in this case. When there are no molecular states between $\mu_{S}$ and $\mu_{D}$, the current will be blocked. Note that the electrochemical potential of the dot changes linearly with $V_{G}$.

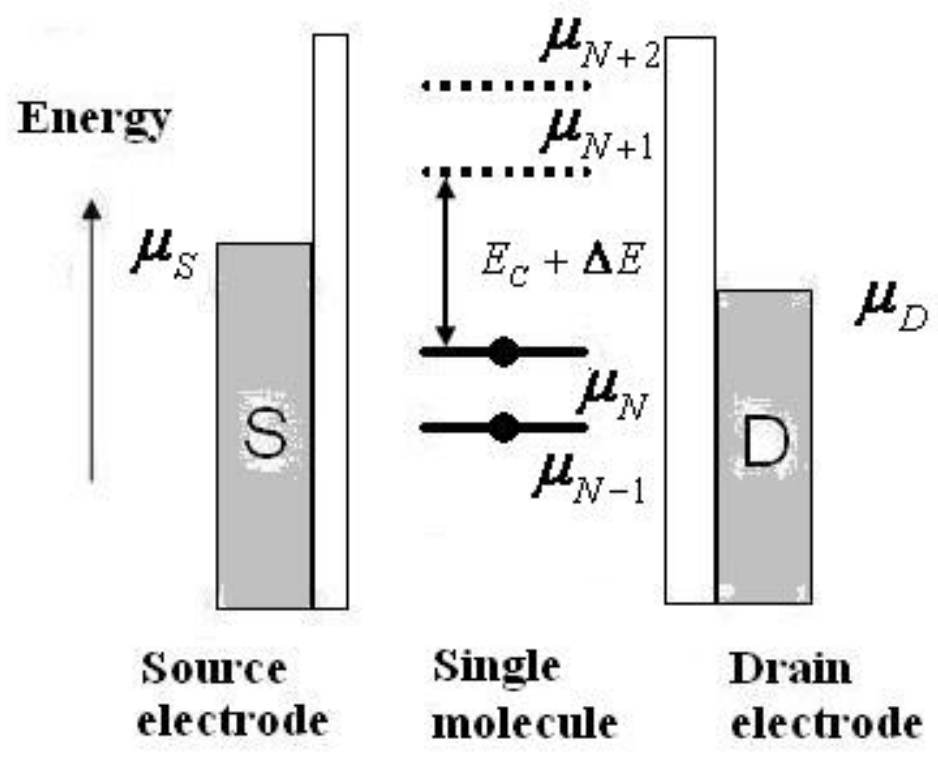

Figure 2.7 Energy diagram of single molecule level between two electrodes.

The electrochemical potential $\mu_{N}$ of a single molecule with $N$ electrons is defined as the minimum energy required to add the $N$-th electron. This energy can be obtained from the energy difference between the total energy $U(N)$ for the $N$ electron state and $U(N-1)$ for the $N-1$ electron state:

$$
\mu_{N} \equiv U(N)-U(N-1) .
$$

It is generally assumed that all electrostatic interactions between the localized electrons in the molecule and all the electrons in the electrodes can be parameterized by the total capacitance $C_{\text {total }}$ of the system, where $C_{\text {total }}$ does not depend on the different 
charged states of the molecule. In this case, the total electrostatic energy for one molecule with $N$ electrons is $Q^{2} / 2 C_{\text {total }}=(N e)^{2} / 2 C_{\text {total }}$. When $N$ electrons reside on the molecule, the total energy is

$$
U(N)=\sum_{i=1}^{N} E_{i}+(N e)^{2} / 2 C_{\text {total }}+\sum_{i=1}^{N} \sum_{j=S, D, G} e_{i, j} V_{d o t} .
$$

After an additional electron is added to the molecule, the total energy increases to

$$
U(N+1)=\sum_{i=1}^{N+1} E_{i}+((N+1) e)^{2} / 2 C_{\text {total }}+\sum_{i=1}^{N+1} \sum_{j=S, D, G} e_{i, j} V_{d o t} .
$$

Here $E_{i}$ is the chemical potential of the molecule with $i$ electrons, which is the energy of the orbital of the molecule that the $i$-th electron would occupy if there was no interaction among electrons. The third term is the effect of three electrodes on the total energy. The electrochemical potential $\mu_{N}$ is

$$
\mu_{N} \equiv U(N)-U(N-1)=E_{N}+(N-1 / 2) e^{2} / C_{\text {total }}+e V_{d o t} .
$$

Here $\mathrm{e} V_{d o t}$ is a function of the source voltage $V_{S}$ and the gate voltage $V_{G}$, which is described by

$$
V_{\text {dot }}=\frac{1}{C_{\text {total }}} \sum_{i-S, D, G} C_{i} V_{i}=\frac{C_{S}}{C_{\text {total }}} V_{S}+\frac{C_{G}}{C_{\text {total }}} V_{G},
$$

where $V_{D}=0$ (the drain electrode is grounded). $C_{\text {total }}$ is the sum of all three capacitances, $C_{\text {total }}=C_{S}+C_{D}+C_{G}$. From the equations above, the electrochemical potential of molecule can be controlled by changing the bias voltage $V$ and/or the gate voltage $V_{G}$ for an arbitrary charge state.

Similarly, the electrochemical potential $\mu_{N+1}$ of the molecule with $N+1$ electrons is

$$
\mu_{N+1} \equiv U(N+1)-U(N)=E_{N+1}+(N+1 / 2) e^{2} / C_{\text {total }}+e V_{d o t} .
$$

This requires $\mu_{N+1}$ to be below $\mu_{S}$ and $\mu_{D}$. Then,

$$
\mu_{N+1}-\mu_{N}=\frac{e 2}{C}+\left(E_{N+1}-E_{N}\right) .
$$

This means that if there are $N$ electrons on a molecule, the external energy required to add one more electron to the molecule is $\mu_{N+1}-\mu_{N}$. 
There are several characteristic energies in this problem:

(1) $\Delta E=E_{N+1}-E_{N}$ : the quantized energy spacing between adjacent electronic states of the molecule.

(2) $E_{C} \equiv \frac{e^{2}}{C}$ : the charging energy required to overcome the Coulomb repulsion among different electrons.

(3) $\frac{e^{2}}{C}+\Delta E:$ the energy required to add an electron to the molecule.

(4) $k_{B} T:$ the thermal energy.

(5) $\gamma \sim h\left(\Gamma_{S}+\Gamma_{D}\right)$ : the intrinsic broadening due to the finite tunneling rate between the molecule and the electrodes.

Single electron transistor behavior can be observed only when the following conditions are satisfied:

(1) $\frac{e^{2}}{C}+\Delta E>>k_{B} T$

(2) $R_{\text {contact }}>>h / e^{2}$, where $R_{\text {contact }}$ is the contact resistance between the molecule and the electrodes and $h / e^{2}$ is the resistance of a single conductance channel.

(3) $\gamma<<k_{B} T$

For condition (1) above, when $\Delta E<k_{B} T$, the system is called a "classical dot", and when $\Delta E>k_{B} T$, it is referred to as a "quantum dot". In the classical dot regime, a tunneling electron can access a continuum of excited states of the dot, and the overall conductance can be described by the tunneling rates averaged over many electronic levels. In the quantum dot regime, each quantum state of the dot can be identified and specific tunneling rates are assigned to each quantum state.

\subsubsection{Single Level Molecule}

For a molecule with a single level, it is assumed that $E_{C}>k_{B} T$ and $\Delta E>k_{B} T$ (the quantum dot regime) and also that $E_{C}$ and $\Delta E$ are large enough that only one additional charge state $(N+1)$ is accessible and that no quantum excited states are 
accessible. Therefore, only two charged states $(N$ and $N+1)$ in their own ground states will be discussed here.

If the electrochemical potential of the $N+1$ electron state $\left(\mu_{N+1}\right)$ when $V=V_{G}=0$ is defined as $E_{0}, \mu_{N+1}$ can be written as

$$
\mu_{N+1}=E_{0}+e V_{d o t} .
$$

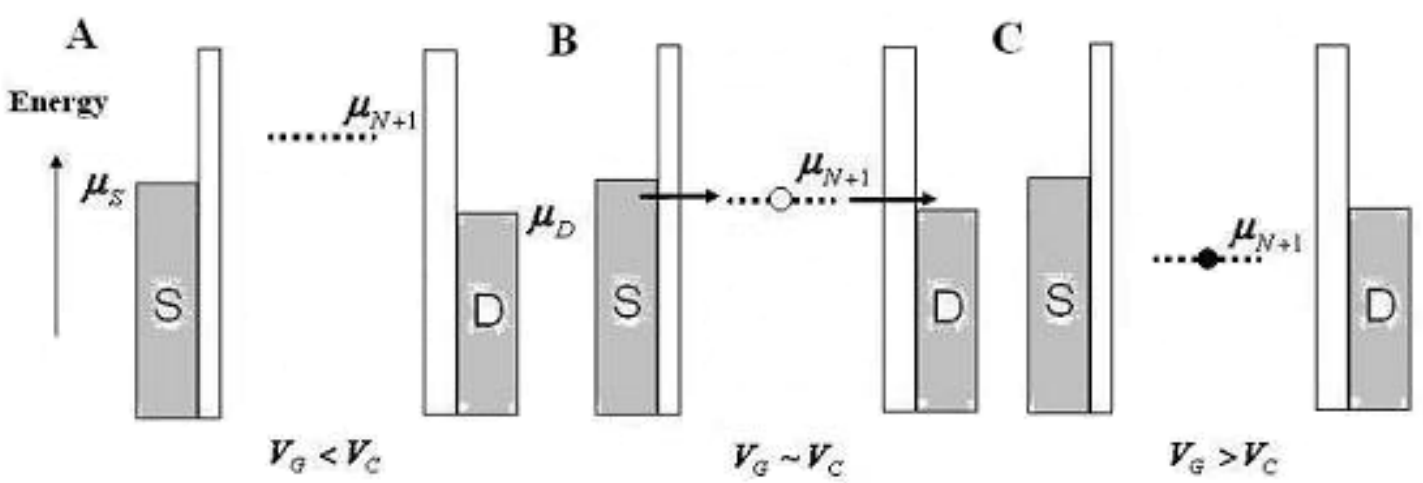

Figure 2.8 The energy diagram of a molecule with a single level.

In Figure 2.8, energy diagrams of a molecule with $E_{0}>0$ and $V \sim 0$ are illustrated. For case A, $V_{G}<V_{C}$, the molecule is always in its $N$ electron state because $\mu_{N+1}$ is above the Fermi level of both the source and drain electrodes. The current will not flow in this case. For case $\mathrm{C},|e| V_{G}>E_{0}\left(C_{\text {total }} / C_{G}\right)$, the current is blocked again because the $N+1$ electron state is always occupied. For case $\mathrm{B},|e| V_{G} \sim E_{0}\left(C_{\text {total }} / C_{G}\right)$, electron transport is allowed because $\mu_{N+1}$ is aligned with the source and drain electrodes. In this case, an electron can jump between the dot and the source or drain electrode freely. The crossing potential $V_{C}$ is defined as $V_{C}=V_{G}$ which corresponds to electrical current flow at low bias $\left(V_{S} \sim 0\right)$, with

$$
V_{C}=\frac{E_{0}}{|e|} \frac{C_{\text {total }}}{C_{G}} .
$$

For $V_{G}=V_{C}$, the two charge states $N$ and $N+1$ of the molecule have the same energy, hence an electron can hop on and off the dot freely. This charge degeneracy of the quantum dot is the origin of the longer relaxation time. The electron can tunnel through the molecule with the molecule remaining in the same intermediate state. 


\subsubsection{Solving Rate Equations for Two-Steps ET}

The rate equation ${ }^{13,36}$ for the transition of an electron into an energy level in a SET is

$$
\frac{d P_{\alpha}}{d t}=\sum_{\beta}\left(\Gamma_{\beta \rightarrow \alpha} P_{\beta}-\Gamma_{\alpha \rightarrow \beta} P_{\alpha}\right),
$$

where $P$ is the probability that the molecule is in a specific state and $\Gamma_{\alpha \rightarrow \beta}$ and $\Gamma_{\beta \rightarrow \alpha}$ are the transition rate between $\alpha$ and $\beta$ states. The current can be calculated by the electron transfer through either of the two tunnel barriers corresponding to the source and drain electrical contacts. In equilibrium the two currents are equal. The current through the source is

$$
I=I_{S}=|e| \sum_{\alpha} \sum_{\beta} \Gamma_{\alpha \rightarrow \beta}^{S} P_{\alpha},
$$

where $e$ is the charge of the electron, $\Gamma_{\alpha \rightarrow \beta}^{S} P_{\alpha}$ is the contribution of the source to $\Gamma_{\alpha \rightarrow \beta}$, multiplied by +1 or -1 depending on the $\Gamma_{\alpha \rightarrow \beta}$ transition contribution to the current.

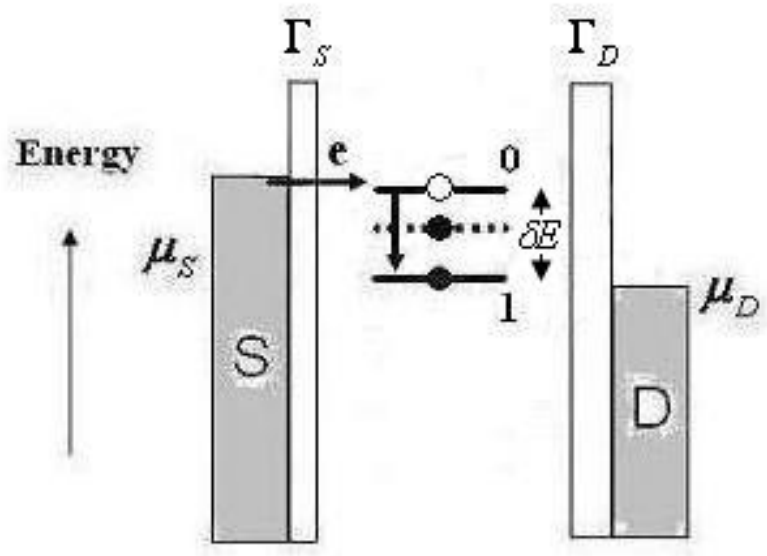

Figure 2.9 Two-step electron transfer by a protein modeled as a quantum dot.

Figure 2.9 is a sketch of the two step tunneling process. The protein's ferric state is labeled by (0) and the ferrous state is labeled (1). $\Gamma_{S}$ and $\Gamma_{D}$ are the tunneling rates between the protein and the source and drain electrodes, respectively. The energy 
difference between the ferric and ferrous states is $\delta E$. Here $P_{0}$ and $P_{1}$ are the probabilities that the level in the quantum dot (protein) is unoccupied and occupied, respectively.

For single electron transport, the total tunneling time $\tau$ can be estimated from $\tau=\frac{|e|}{I}$, where $\tau \sim$ ps for a current $I \sim 10^{-7} \mathrm{~A}$. The rate equations for two-step electron transfer are set up by making the following assumptions:

(1) When the electron tunnels into the protein, the energy level 0 relaxes to level 1 via a structural distortion in a time $\sim \tau$.

(2) The process is not reversible and fluctuations between levels 1 and 0 resulting from nuclear distortions are neglected. This is especially true at low temperatures where the thermal energy is much smaller than the vibrational energy of the heme group $\left(k_{B} T<<\right.$ $E_{\mathrm{vib})}$.

With these assumptions, the time change rate of $P_{0}$ can be written as

$$
\begin{aligned}
& \frac{\partial P_{0}}{\partial t}=-P_{0}\left(\Gamma_{S} f_{S}^{0}+\Gamma_{D} f_{D}^{0}\right)+P_{1}\left[\Gamma_{S}\left(1-f_{S}^{1}\right)+\Gamma_{D}\left(1-f_{D}^{1}\right)\right] \\
& \frac{\partial P_{1}}{\partial t}=P_{0}\left(\Gamma_{S} f_{S}^{0}+\Gamma_{D} f_{D}^{0}\right)-P_{1}\left[\Gamma_{S}\left(1-f_{S}^{1}\right)+\Gamma_{D}\left(1-f_{D}^{1}\right)\right]=-\frac{\partial P_{0}}{\partial t}
\end{aligned} .
$$

where $f_{S}^{0(1)}$ and $f_{D}^{0(1)}$ are the Fermi distribution functions of the source and drain electrodes, respectively, for the unoccupied (occupied) energy levels. These can be written as

$$
\begin{gathered}
f_{S}{ }^{0}=\left[1+\exp \left(\frac{\mu_{N+1}-\mu_{S}}{k_{B} T}\right)\right]^{-1} \\
f_{S}{ }^{1}=\left[1+\exp \left(\frac{\mu_{N+1}-\delta E-\mu_{S}}{k_{B} T}\right)\right]^{-1} \\
f_{D}{ }^{0}=\left[1+\exp \left(\frac{\mu_{N+1}-\mu_{D}}{k_{B} T}\right)\right]^{-1} \\
f_{D}{ }^{1}=\left[1+\exp \left(\frac{\mu_{N+1}-\delta E-\mu_{D}}{k_{B} T}\right)\right]^{-1} .
\end{gathered}
$$


Here $\mu_{N}$ is the chemical potential of the system with $N$ electrons and $\mu_{N+1}$ is the chemical potential when an additional electron is added to the system. Using the Eqs. (2.2) and (2.1), the chemical potential of the $N+1$ electron state can be written as

$$
\mu_{N+1}=E_{0}-|e| V_{d o t}=E_{0}-|e| \frac{C_{G} V_{G}+C_{S} V_{S D}}{C_{\text {total }}},
$$

where the energy $E_{0}=e V_{C} C_{G} / C_{\text {total }}$ (from the Eq. 2.3) is proportional to the crossing potential $V_{C}$.

In equilibrium, $\frac{\partial P_{0}}{\partial t}=\frac{\partial P_{1}}{\partial t}=0$ and $P_{0}+P_{1}=1$. The current is calculated by

$$
\frac{I}{|e|}=-P_{0} \Gamma_{S} f_{S}+P_{1} \Gamma_{S}\left(1-f_{S}\right) .
$$

Simplifying, one obtains

$$
\begin{aligned}
& P_{0}=\frac{\Gamma_{S}\left(1-f_{S}^{1}\right)+\Gamma_{D}\left(1-f_{D}^{1}\right)}{\Gamma_{S}\left(1+f_{S}^{0}-f_{S}^{1}\right)+\Gamma_{D}\left(1+f_{D}^{0}-f_{D}^{1}\right)} . \\
& \frac{I}{|e|}=\Gamma_{S}\left[1-f_{S}^{1}-P_{0}\left(1+f_{S}^{0}-f_{S}^{1}\right)\right]
\end{aligned} .
$$

In the calculation shown in Fig. 2.10(a), the differential conductance $d I / d V$ was calculated by numerically taking the derivative of Eq. 2.4 ( $I$ as a function of the drainsource voltage $V$ ) with respect to $V$. As in the experiments, the chemical potential of the drain was set to $\mu_{D}=0$ (i.e., the drain contact was grounded). Note that if $\delta E=0$ there is no two-step process and the result of a single-level quantum dot is recovered (shown in Fig. 2.10(b)).
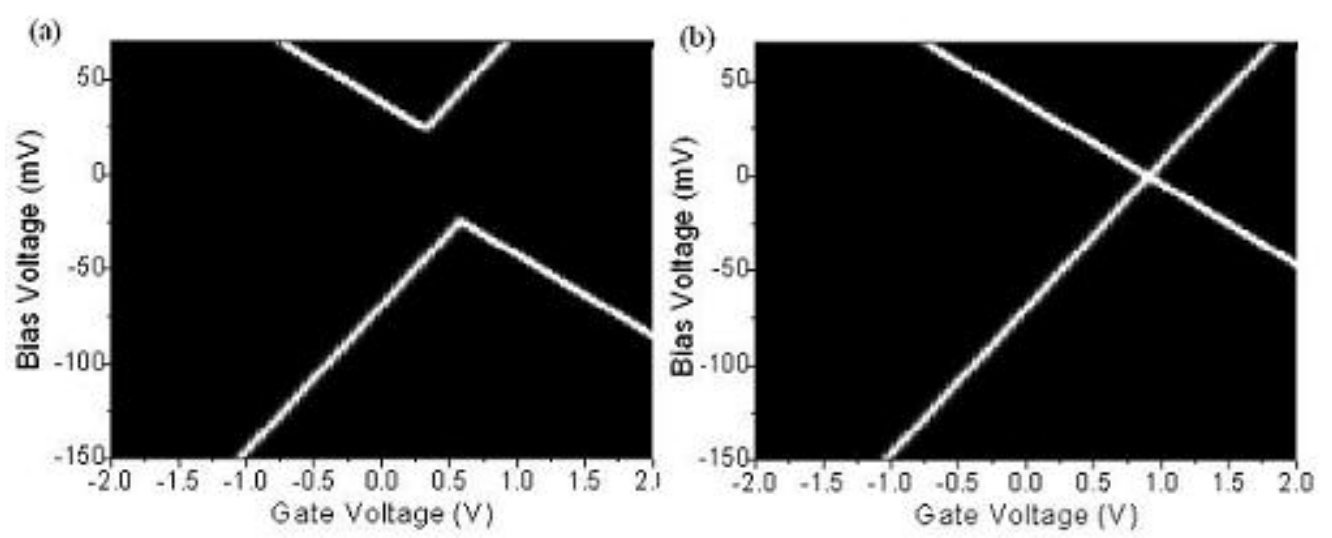

Figure 2.10 The calculation results for (a) with two-step process at $T=6 \mathrm{~K}, \delta E=25 \mathrm{mV}, E_{0}=25 \mathrm{mV}$, $C_{D}: C_{S}: C_{G}=12: 23.5: 1$, tunneling rates $\Gamma_{S}=\Gamma_{G}=10 \mathrm{GHz}$ (b) without two-step process at $T=6 \mathrm{~K}, \delta E=0$ $\mathrm{mV}, E_{0}=25 \mathrm{mV}, C_{D}: C_{S}: C_{G}=12: 23.5: 1$, tunneling rates $\Gamma_{S}=\Gamma_{G}=10 \mathrm{GHz}$. 


\subsection{The Kondo Effect}

The Kondo effect is a consequence of the interaction between a magnetic impurity with a conduction electron. ${ }^{37}$ It was first explained by Jun Kondo in 1964 for the effect of magnetic impurities in metals. ${ }^{38}$ The interacting electrons confined on a quantum dot between two electrodes can be described by Phil Anderson's model. ${ }^{39}$ Figure 2.11 shows the relation of conductance verse temperature for the Kondo effect in quantum dots. ${ }^{40}$ The red line shows the Kondo effect at low temperature for the odd number of electron confined in the dot. The blue line shows the case where the dot contains an even number of electrons and the total spin is zero, and thus the Kondo effect does not occur.

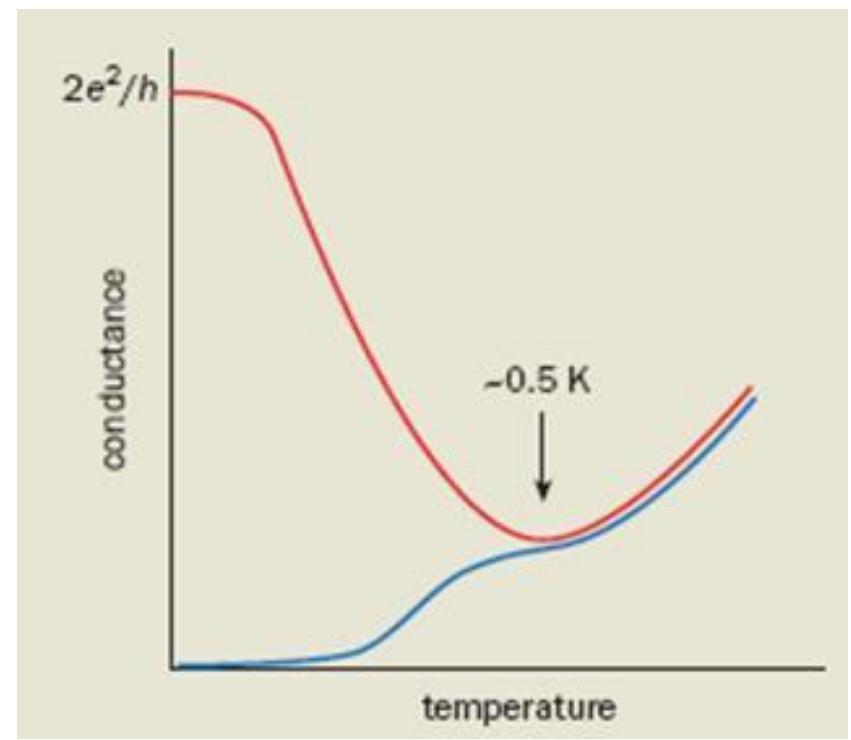

Figure 2.11 The Kondo effect in quantum dots. The red line show the Kondo effect for the odd number of electron confined in the dot. The blue line show the case that the Kondo effect does not occurs (from Ref. 40).

The Kondo effect has captured the interest of both experimentalists and theorists with the rapidly development of nanotechnology. Various groups around the world have exploited trapping a molecule between two metal electrodes to make single-electron transistor $^{24-26}$ and some of these devices exhibit the Kondo effect ${ }^{24,26}$ Figure 2.12 shows the Kondo effect from the devices made using a relatively short molecule, $\left[\mathrm{C}_{0}\right.$ (tpy$\left.\mathrm{SH})_{2}\right]^{2+} .^{24}$ The Kondo effect is observed as an enhanced conductance at zero bias resulting from the formation of a bound state between a local spin in the molecule and the 
conduction electrons in the electrodes. ${ }^{24}$ The graph on the left of Fig. 2.12 shows that the peak height decreases with temperature. The graph on the right shows that peak splits with increasing magnetic field. These are characteristic properties of the Kondo effect in quantum dots that must be verified in order to unambiguously identify its presence. Normally it can only be observed if the electron maintains its spin coherence during the tunneling process and if the electronic state of the resonant energy level has a net spin.

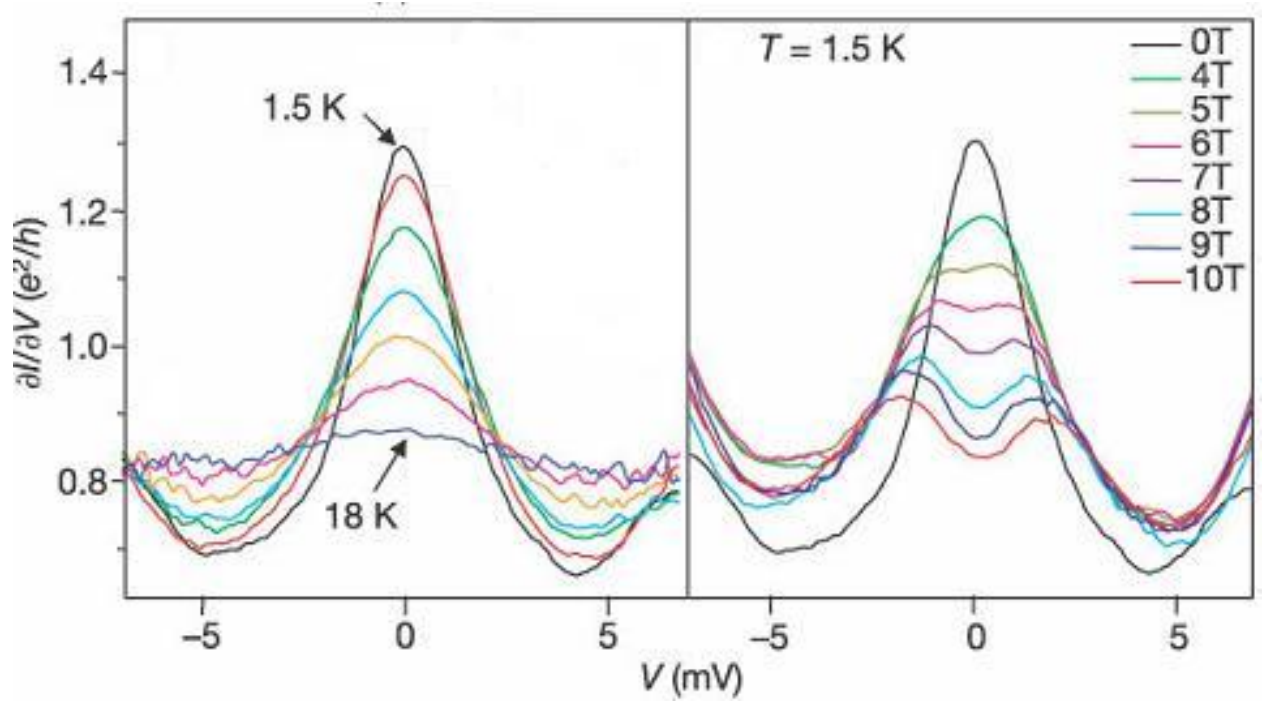

Figure 2.12 The Kondo effect from devices made using the shorter molecule, $\left.\left[\mathrm{C}_{\mathrm{o}} \text { (tpy-SH }\right)_{2}\right]^{2+}$. Left graph show the temperature dependence of the Kondo peak. Right graph show the magnetic dependence of the Kondo peak (from Ref. 24).

\subsection{Summary}

In this chapter basic tunneling physics phenomena were introduced, including inelastic electron tunneling, elastic electron tunneling and resonance electron tunneling. Then, single electron transistor theory for the single-level molecule was discussed, including the rate equation for two-step ET process. Then the Kondo effect was reviewed. These are the basic theories for discussing electron transfer by a single protein in the next chapters. 


\section{Chapter 3}

\section{Device Fabrication}

\subsection{Introduction}

Various experimental devices have been developed to measure the conductance of molecules. $^{41}$ One of the most powerful methods is the EC-STM technique discussed in the Chapter 1. One advantage of this technique is that the proteins can be imaged before measuring them. However, its energy resolution is usually poorer than other techniques and it lacks the independent gate electrode, which is a significant disadvantage when one wishes to study charge states of the molecule.

It is difficult to fabricate nanometer gap electrodes directly on an insulating surface with electron beam lithography because of poor reproducibility at the nanometer scale. A method used to fabricate such electrodes is the mechanical break junction technique. ${ }^{42}$ The disadvantage of the method is that it lacks a gate electrode due to experimental limitations. Other nanofabrication techniques include electrodeposition ${ }^{43}$ and nanopore techniques. ${ }^{44}$ The technique used in our experiment is the electromigration technique. ${ }^{45}$ In this chapter, various experimental issues regarding device fabrication will be discussed. The junction fabrication procedure and the protein deposition and measurement setups will be discussed.

\subsection{Fabrication of Junctions}

Commercial silicon wafers with a $200 \mathrm{~nm}$ thick layer of $\mathrm{SiO}_{2}$ on top and a resistivity ranging from 0.002 to $0.003 \Omega-\mathrm{cm}$ were used. The Si wafers were $550 \mu \mathrm{m}$ thick. Gate failure and leakage were caused by impurities and pin-holes present in the gate oxide. In our experiments, the $200 \mathrm{~nm}$ thickness of $\mathrm{SiO}_{2}$ usually failed while soldering of the contacts by hand. Therefore, all the gates were checked to be good insulators up with a gate voltage of at least $5 \mathrm{~V}$ before cooling down. The electrodes were fabricated by standard lithography techniques. ${ }^{46}$

\subsubsection{Photolithograph}


First 68 chips were fabricated on a single 4 -inch $\mathrm{Si} / \mathrm{SiO}_{2}$ wafer using standard photolithography (Fig. 3.1). Eight microscopic electrodes were designed on each chip (5 $\mathrm{mm}$ by $5 \mathrm{~mm}$ ) so that it would fit in the sample holder of the cryostat in our experiments (Fig. 3.2). The thickness of the evaporated pattern was approximately $3 \mathrm{~nm}$ for $\mathrm{Cr}$ and 90 $\mathrm{nm}$ for Au. This total thickness was enough for the macroscopic electrodes to stick to the junctions easily. The design pattern of the macroscopic electrodes is shown in Figure 3.3(A). The pattern consists of a long vertical electrode that serves as the common drain electrode for all the junctions, and another long vertical electrode that serves as the common gate electrode. There are six other macroscopic electrodes that serve as source electrodes that share the same drain and gate electrodes.

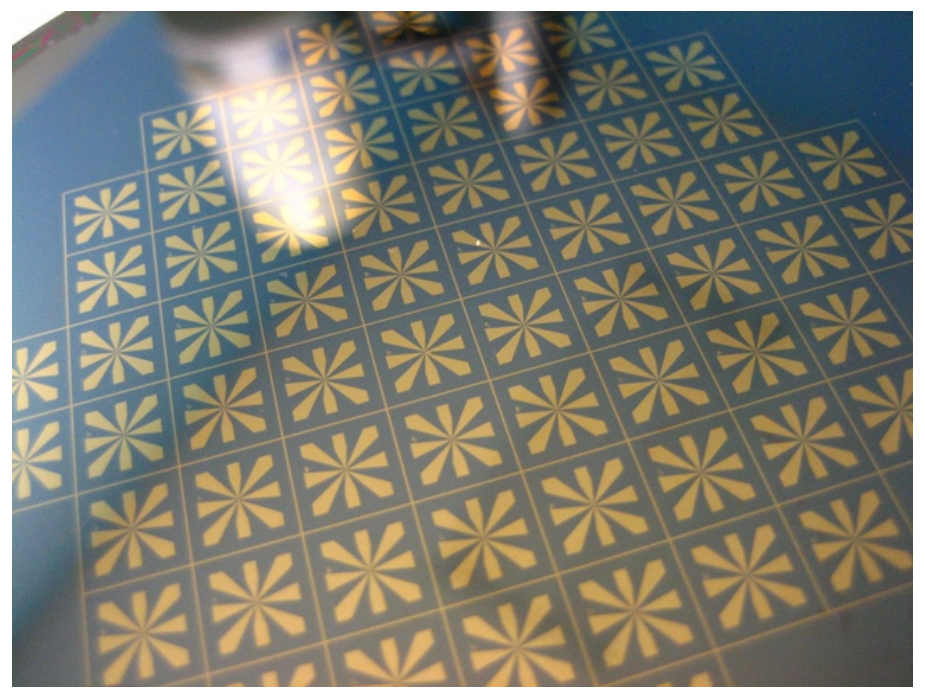

Figure 3.1 There are 68 chips on 4 inch silicon wafer.
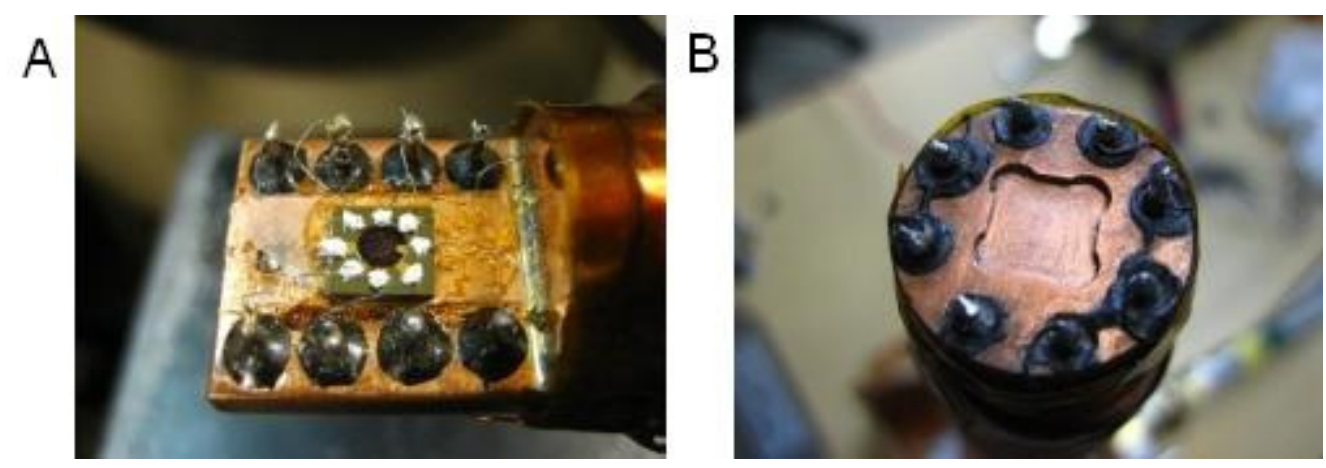

Figure 3.2 The sample holder in the cryostat. (A) in ARS, Helitran LT-3-110 open cycle cryostat. (B) in ARS, Displex DE-202 Closed Cycle cryostat. 


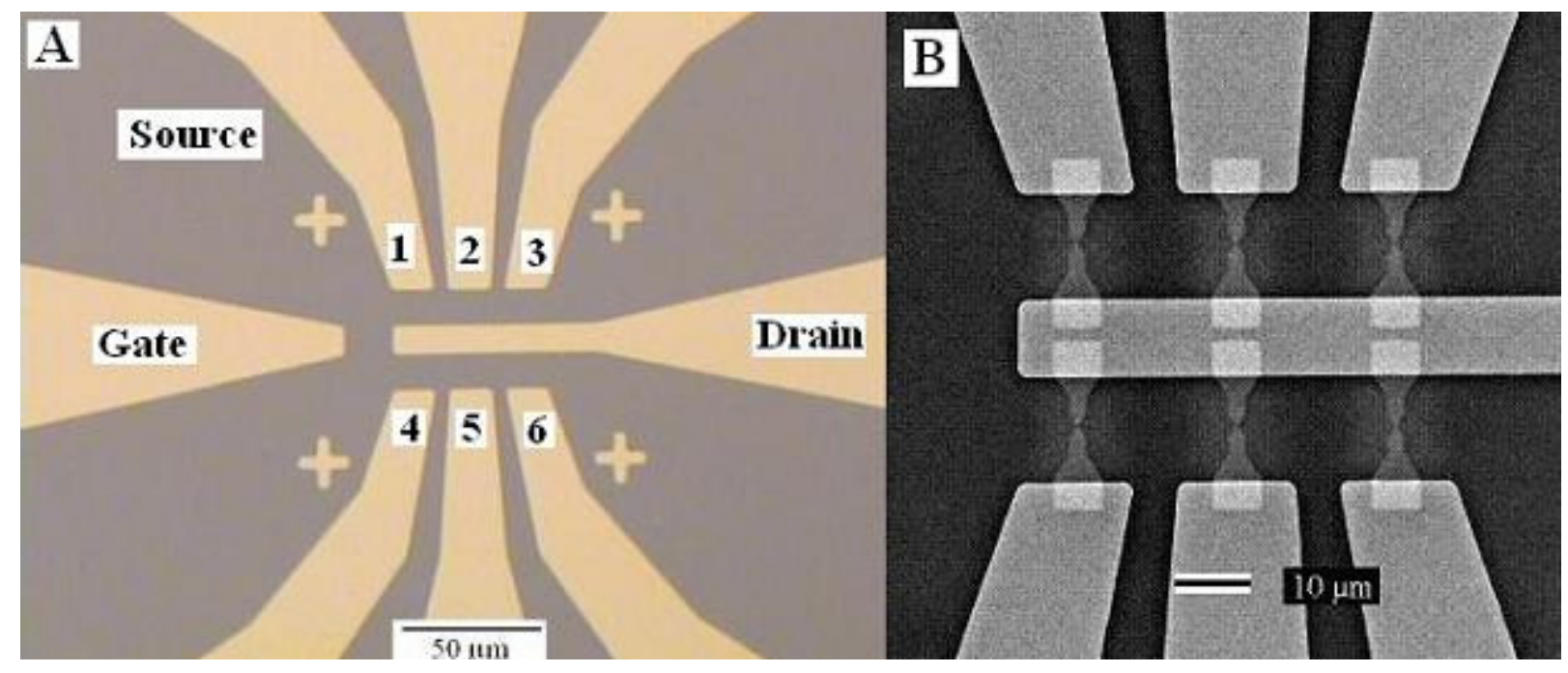

Figure 3.3 A: An optical microscope image of a chip with 8 electrodes. B: An SEM micrograph of 6 junctions after e-beam lithography and lift-off.

\subsubsection{Electron Beam Lithography}

A chip that has six junctions is shown in Figure 3.3(B) (scanning electron microscope image). The junctions were fabricated using $e$-beam lithography. A bilayer of $e$-beam resist (first MMA $120 \mathrm{~nm}$ thick and then PMMA $180 \mathrm{~nm}$ thick) was spun on the surface. Then the target area that would later become the junctions was exposed using the electron beam (dose approximately $200 \mu \mathrm{C} / \mathrm{cm}^{2}$ ). The development time was between 20 and 45 seconds using the standard PMMA developer (MIBK:IPA 1:3). After development, platinum films (10 to $20 \mathrm{~nm}$ thick) were deposited on a $\mathrm{Si} / \mathrm{SiO}_{2}$ substrate by magnetron sputtering with approximately a $15^{\circ}$ degree deposition angle with respect to the surface normal of the sample. The samples were rotated during deposition in order to improve thickness homogeneity. The deposition rate was $0.28 \AA / \mathrm{s}$ and the base pressure was $5 \times 10^{-8}$ Torr. The width of junctions was usually between $100 \mathrm{~nm}$ and $300 \mathrm{~nm}$. The total resistance of the junction (including parasitic resistance) at room temperature ranged between $100 \Omega$ and $1 \mathrm{k} \Omega$. Compared to gold, Pt sticks to $\mathrm{SiO}_{2}$ very well without Ti or Cr adhesion underlayers.

\subsection{Breaking Junctions by Electromigration}


In most cases, $\mathrm{Pt}$ junctions were broken by electromigration at voltages ranging from $0.6 \mathrm{~V}$ to $1.0 \mathrm{~V}$ with feedback and a current between approximately $2 \mathrm{~mA}$ and $7 \mathrm{~mA}$ at temperatures below $80 \mathrm{~K}$ (depending on the experiment). The failure current density for a $\mathrm{Pt}$ junction was approximately $10^{12} \mathrm{~A} / \mathrm{m}^{2}$. In order to break the junction, a bias voltage was ramped at a rate of $30 \mathrm{mV} / \mathrm{s}$ and the computer was programmed to stop when the current dropped by a certain percentage, approximately $10 \%$ for the most successful samples. Usually the junctions needed to be broken several times until conductance peaks were found in some samples coated with the protein solution. The voltage ramping rate was slower $(10 \mathrm{mV} / \mathrm{s})$ after the first break in order to control the gap between electrodes. In this case, the gap of electrodes was usually approximately $5 \mathrm{~nm}$ wide after breaking junction several times at low temperature and warming up (Fig. 3.4(A)). Breaking the junction without feedback (the program was stopped by hand after a drop in the current was observed) caused large gaps and was not practical.
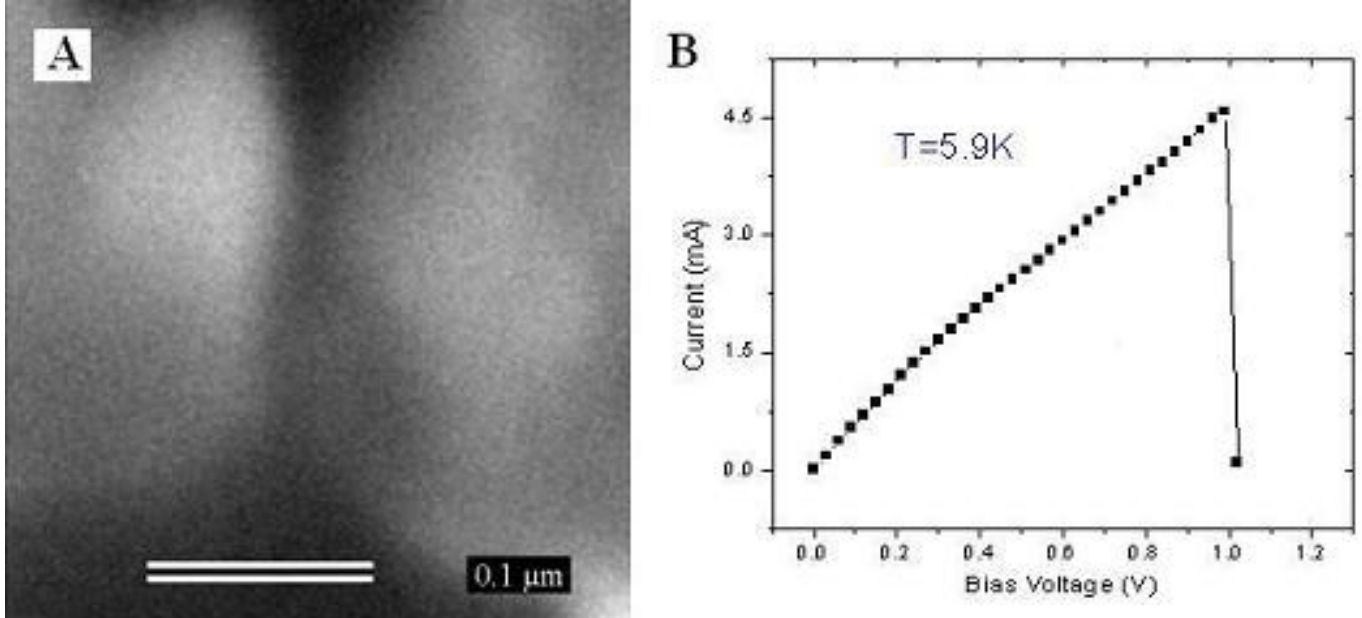

Fig. 3.4 Breaking junction by electromigration at cryogenic temperature. A: the gap after braking junction. B: I-V curve during breaking junction.

Figure 3.4(B) shows the current versus the total bias voltage applied to the circuit during the process of breaking the junction. In this plot, the current keeps increasing until $V_{\text {bias }}$ reaches $1.02 \mathrm{~V}$ and then it suddenly drops to approximately zero while ramping at a constant rate of $30 \mathrm{mV} / \mathrm{s}$. This breaking curve shows that the junction behaves like an ohmic conductor up to a certain current density and then fails, forming a physical gap within the junction. 
In most cases, junctions needed to be broken several times until they were broken completely. The gap of broken junction by electromigration were observed in images taken by SEM or AFM. The presence of a gap between the electrodes is clearly visible after the wire is broken and the size of the narrowest gap is around $5 \mathrm{~nm}$. However, an accurate measurement of the gap size is difficult because the resolution limit of the SEM.

\subsection{Protein Deposition}

Because the gap of a broken junction is $\sim 5 \mathrm{~nm}$, which is the approximate diameter of the proteins studied here, these electrodes can be used to wire up single proteins in our experiments. The molecular deposition method used to accomplish this objective differs from molecule to molecule and will be discussed in the Chapter 5. The procedure is summarized below.

First, junctions were rinsed with optima acetone and isopropanol, and subsequently cleaned with $\mathrm{O}_{2}$ plasma in order to remove possible contaminants on the electrode surface. After the cleaning procedure, the protein solution was dropped on the samples. The samples were kept at $4{ }^{\circ} \mathrm{C}$ in order to allow $\mathrm{H}_{2} \mathrm{O}$ to evaporate slowly and minimize damage to the protein. Finally the junctions coated with proteins were loaded into a cryostat and broken by the electromigration process to form a tunnel gap at cryogenic temperatures ( $77 \mathrm{~K}$ or $5 \mathrm{~K}$ ). Proteins deposited on the surface of junctions before the breaking that end up in the gap were identified by subsequent conductance measurements.

Electrical measurements were performed immediately after breaking the junction by electromigration at cryogenic temperatures. The conductance $\left(d I / d V_{\text {bias }}\right)$ as a function of bias and voltages was acquired by numerically differentiating $I-V_{\text {bias }}$ curves. It was verified that the same results were obtained using a direct measurement technique utilizing a lock-in amplifier.

The effects of a protein in the gap were occasionally observed during the breaking process (Figure 8.6(a)). This resulted in a significant increase in the current even after the junction was broken. The mechanism of incorporation of proteins within the gap is not well understood. One possible explanation is the electrostatic trapping mechanism. ${ }^{13}$ 


\subsection{Measurement Setup}

Most transport measurements and the electromigration process were performed in two ARS(Advanced Research System, Inc.) cryostats, a Helitran LT-3-110 open cycle cryostat (Fig.3.5(a)) and a Displex DE-202 Closed Cycle cryostat (Fig. 3.5(b)). The standard measurement devices were two Keithley source meters, a Keithley 6430 with preamplifier provide bias voltage and measure current and a Keithley 2400 used to provide the gate voltage.
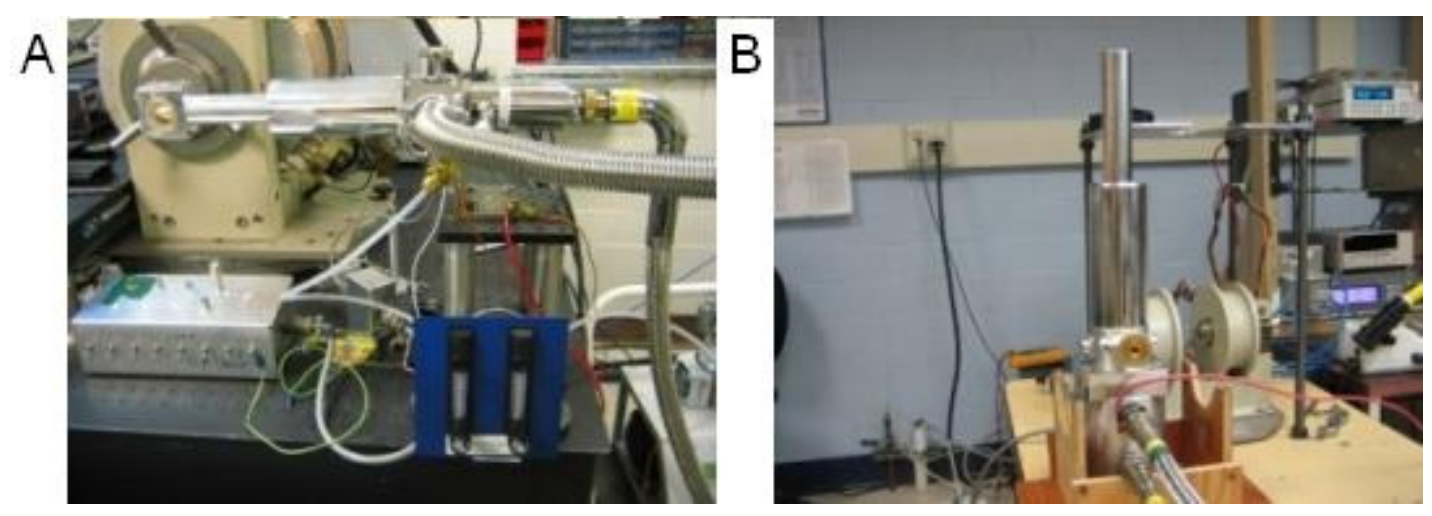

Fig. 3.5 The two cryostat used for low temperature conductance measurements. (A) ARS, Helitran LT-3110 open cycle cryostat (B) ARS, Displex DE-202 Closed Cycle cryostat.

\section{Software}

All junctions were broken in this work were taken using a Labview program developed for this purpose called "Breaking junction with Feedback" (Figure 3.6). Another program called "I-V with Keithley 6430" can control two voltage outputs, which are usually used for controlling the source-drain bias and the gate bias (Fig. 3.7). It can also read several analog signals and record them in real time. It is a very versatile program and has many useful functions especially developed for three terminal transport measurements. 


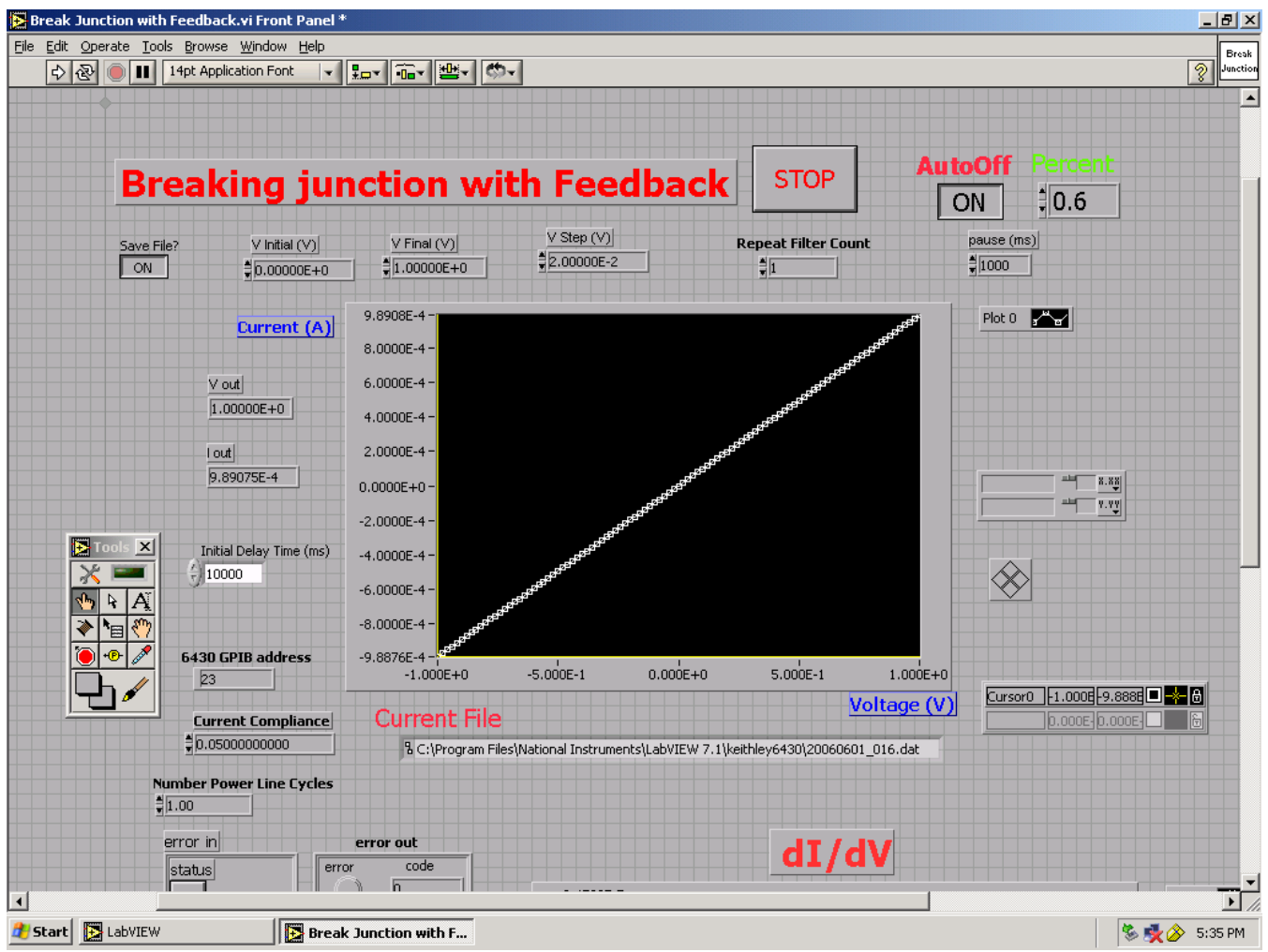

Figure 3.6 The program of breaking junction with feedback.

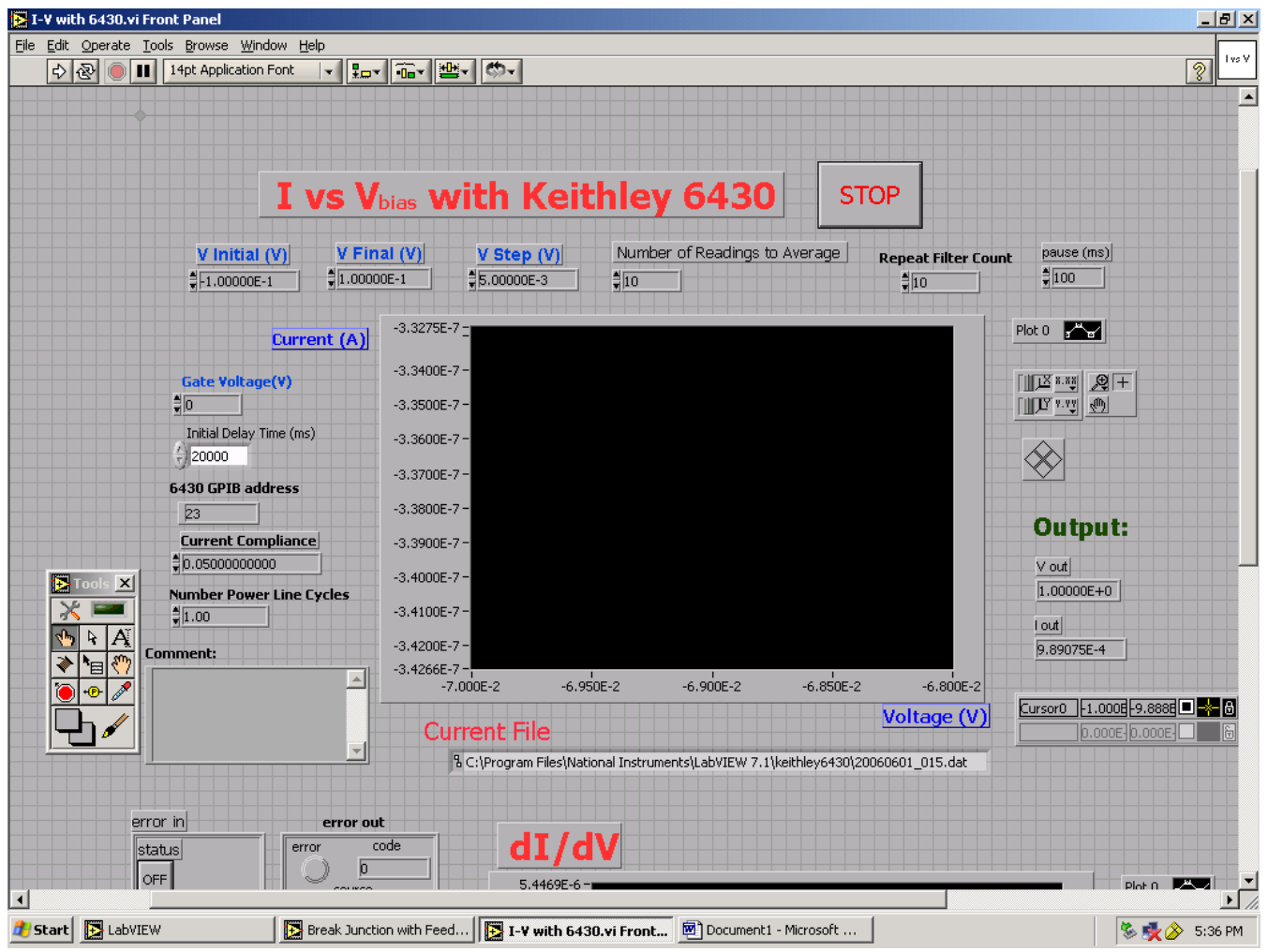

Figure 3.7 The program of current vs. bias. 


\subsection{Summary}

In this chapter, various issues concerning the device design, device fabrication, sample preparation and the measurement setup procedures were discussed. The junction cleaning process and protein deposition is a critical process for successful device fabrication and requires careful control. Finally the electromigration process is used to form two electrodes with a several nanometer sized gap that can be bridged by single proteins previously deposited on the surface. As discussed in the next chapters, conductance measurements after the junction breaking process show simple tunnel $I-V$ curves or the Coulomb blockade depending on whether a protein is located in the gap or not. 


\section{Chapter 4}

\section{Control Experiments in Blank Junctions}

\subsection{Introduction}

As discussed in the Chapter 3, different methodologies have been developed to measure the conductance of single molecules. Each of them has its advantages and disadvantages. EC-STM offers mechanical control, but the incorporation of a third, independent gate electrode is difficult to realize. Three-terminal molecular junctions have been made using standard (e-beam) fabrication techniques. The electromigration fabrication technique has the disadvantage that small metal grains can form near the gap region masking molecule information. The local temperature to which metal junctions become heated during the electromigration process is not well known. Despite these shortcomings, many groups around the world are using this method to perform experiments on single molecule transport.

In this chapter, several pitfalls and ways of distinguishing the molecular transport from impurity transport will be discussed.

\subsection{The Origins of Conductance Peaks}

There are several possible origins for conductance peaks. One possibility is that they come from the molecule that one intends to measure, which has discrete energy levels. Another possibility is that an impurity in the junction can cause spurious signals. This impurity can consist of metal grains produced during junction-breaking process by electromigration. ${ }^{47,48,49}$ It is therefore necessary to know the behavior of metal grains first.

\subsection{The Pt Grains Produced During Electromigration}

In control experiments, 107 blank Pt junctions were broken by electromigration at a temperature of $77 \mathrm{~K}$. The broken junctions had a resistance is ranging from $1 \mathrm{M} \Omega$ to 1 $\mathrm{G} \Omega$ at $77 \mathrm{~K}$. All but one of the broken junctions did not show conductance peaks. Figure 4.1(a) is a typical result (differential conductance $d I / d V_{\text {bias }}$ versus $V_{\text {bias }}$ ) from a 
blank Pt measured at $T=77 \mathrm{~K}$. The conductivity was relatively low and there was a significant amount of noise for these structures. Only one sample had conductance peaks as shown in Fig. 4.1(b).
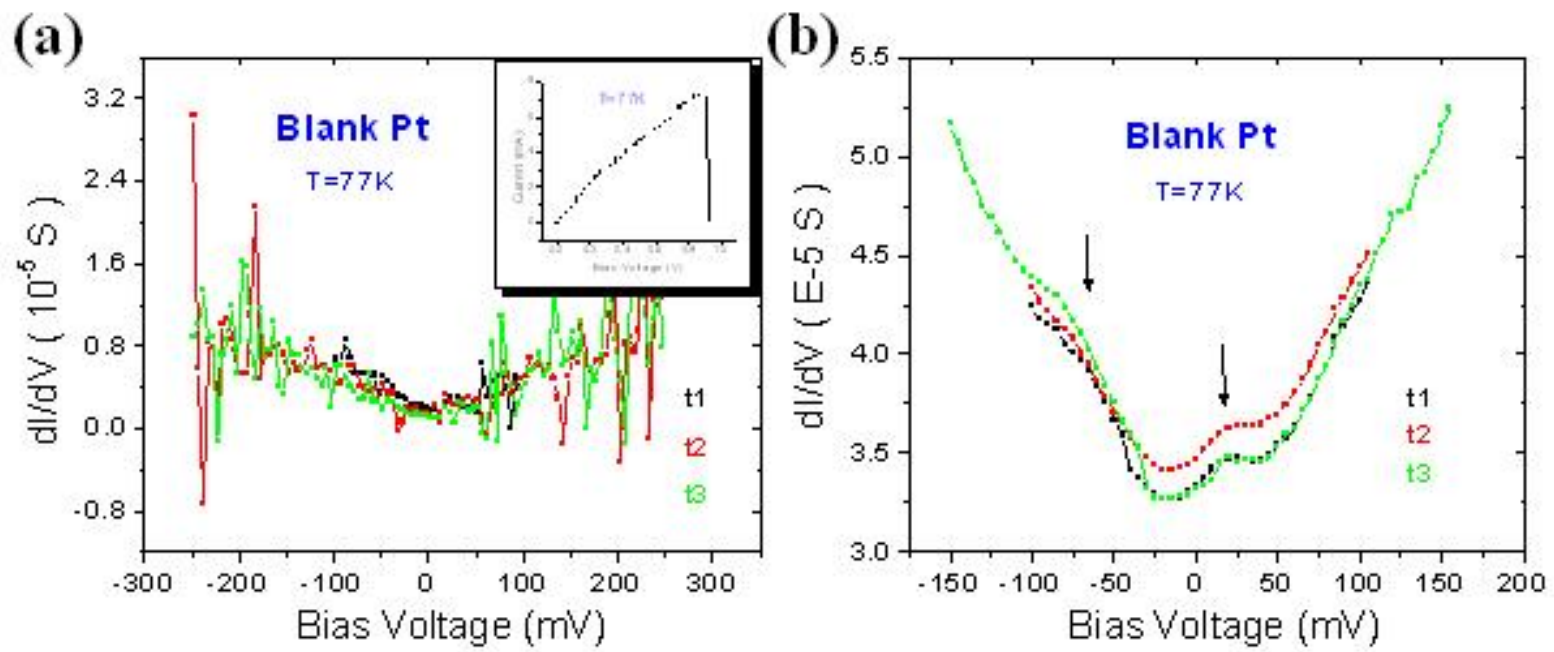

Figure 4.1 Two blank Pt junctions at $77 \mathrm{~K}$. (a) is a typical result (differential conductance $d I / d V_{\text {bias }}$ versus $\left.V_{\text {bias}}\right)$. The inset shows the current during the junction-breaking process. (b) is the only one sample with conductance peaks.

In addition, 31 bare Pt junctions were broken by electromigration and measured at $5.5 \mathrm{~K}$. Seventeen of these did not have peaks in the conductance vs. bias voltage as shown in Fig. 4.2(a). Out of the remaining 14 junctions that had conductance peaks, 11 broken junctions did not have a dependence on the gate voltage. The remaining three junctions did have conductance peaks whose positions were dependent on the gate voltage. Coulomb diamonds, characteristic of SET behavior, were observed in the conductance as a function of gate and bias voltages as shown in Fig. 4.2(b)(c). This demonstrates that occasionally Pt grains are formed during electromigration at $\sim 5 \mathrm{~K}$, just like gold grains can form during electromigration, which can mask the desired molecular information. ${ }^{47,48,49}$ The electronic behavior of small metal grains between the electrodes can be described using the theory of quantum dots. ${ }^{50}$ These islands have several energy levels and the conductance peaks from metal grains can be tuned by gate voltage modulation. More Coulomb diamonds can be expected in $d I / d V$ and all the degeneracy points are around zero bias. If there is one island, all diamond edges are linear and have two kinds of slopes whose values are determined by the three capacitances associated with each electrode in the transistor geometry. ${ }^{13,51,52}$ If there is more than one island, 
more complicated Coulomb diamond shapes and more than two kinds of slopes may be found. ${ }^{53}$

In order to check whether Pt grains can be formed at $77 \mathrm{~K}$, four blank Pt junctions were broken by electromigration at $77 \mathrm{~K}$ and then cooled down to $5 \mathrm{~K}$. None of these junctions had a discernible conductance peak at $77 \mathrm{~K}$. After cooling down to $5 \mathrm{~K}$, two of these broken junctions had conductance peaks (shown in Fig. 4.2(d)). Out of these two samples, one had a gate voltage effect and the other did not. This shows that Pt grains can form at $77 \mathrm{~K}$ in some cases and that their conductance peaks can't be observed because of the high temperature.

(a)

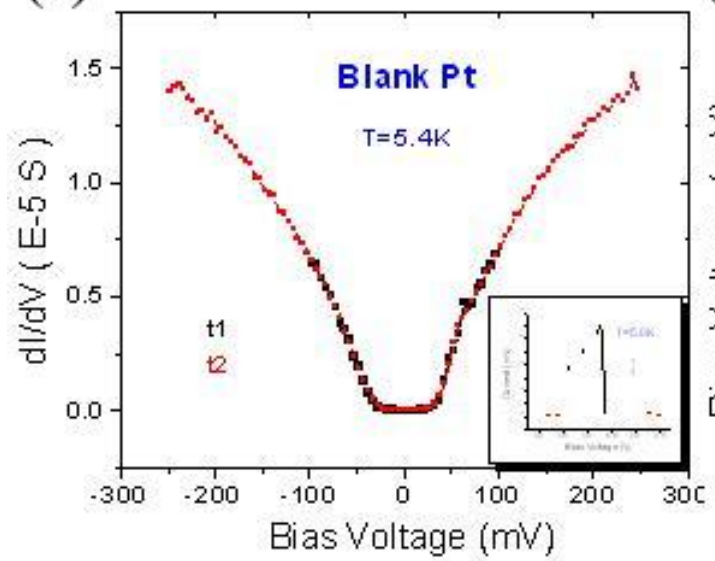

(c)

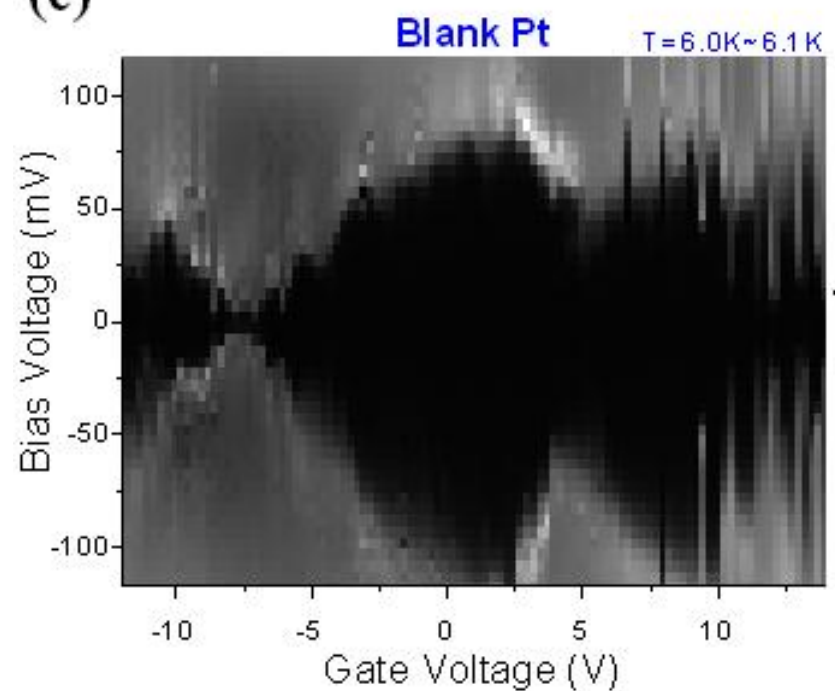

(b)

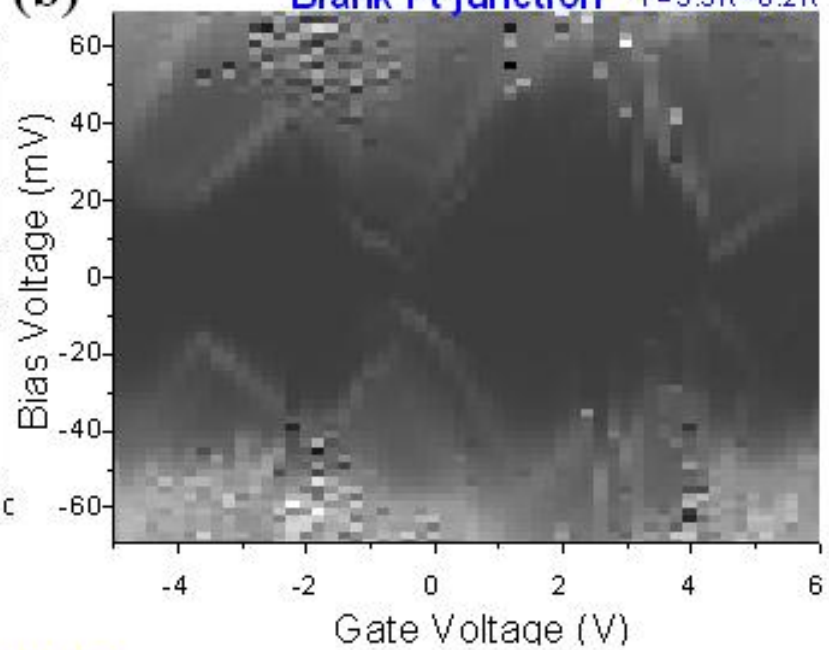

(d)

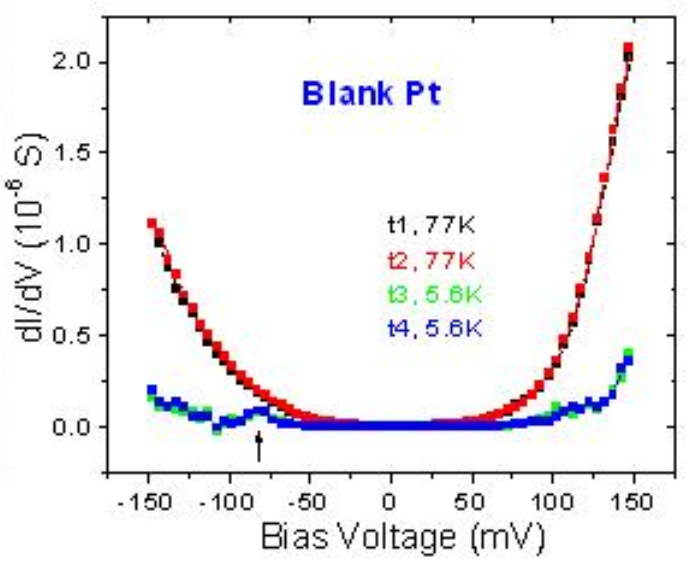

Figure 4.2 Measurements of blank Pt junctions at 5 6 K. (a) A sample that did not have peaks in the conductance vs. bias voltage. The inset shows the junction-breaking process. (b) One Pt junction with Coulomb diamonds, characteristic of SET behavior, was observed in the conductance as a function of gate and bias voltages. (c) Another sample with Coulomb diamonds, characteristic of SET behavior. (d) Conductance of blank Pt junction was broken by electromigration at $77 \mathrm{~K}$ and then cooled down to $5 \mathrm{~K}$. 
Although a more detailed analysis of these data could be made, the focus here is on the samples containing proteins, and therefore the aim is simply to point out the importance of being able to distinguish the conductance due to the presence of the protein from artifact arising from the fabrication of the junctions.

\subsection{The Behavior of Metal Grains in the Gap of Electrodes}

In this section, some results of the gold grains from other groups will be introduced in order to understand the behavior of metal grains in the gap of electrodes.

\section{(1) No Grain or Single Grain}

Instead of breaking a Au junction, the Au electrodes with nanometer gap can be fabricated at $4 \mathrm{~K}$ by depositing at an angle through a mask on a substrate and placing the evaporator in a helium cryostat. ${ }^{54}$ The gap can be controlled by tilting the deposition angle. ${ }^{55}$ Figure 4.3 shows the fabrication and transport properties of metallic nanogaps ( $T$ $=4.2 \mathrm{~K}$ ). When a single gold grain is left between the source and drain electrodes (Fig. 4.3(a)), the corresponding differential conductance plot has Coulomb diamonds (Fig. 4.3(d)); when the grain is attached to one of the leads (showed in Fig. 4.3(b)) by changing the deposition conditions, the differential conductance does not depend on the gate voltage (Fig. 4.3(e)).

The experimental platform introduced above allow the nanometer electrodes to be fabricated at low temperature and in high vacuum. And single molecules can be evaporated into the gap of the electrodes without changing any conditions after the electrodes is fabricated. This method can provide a clean background. 


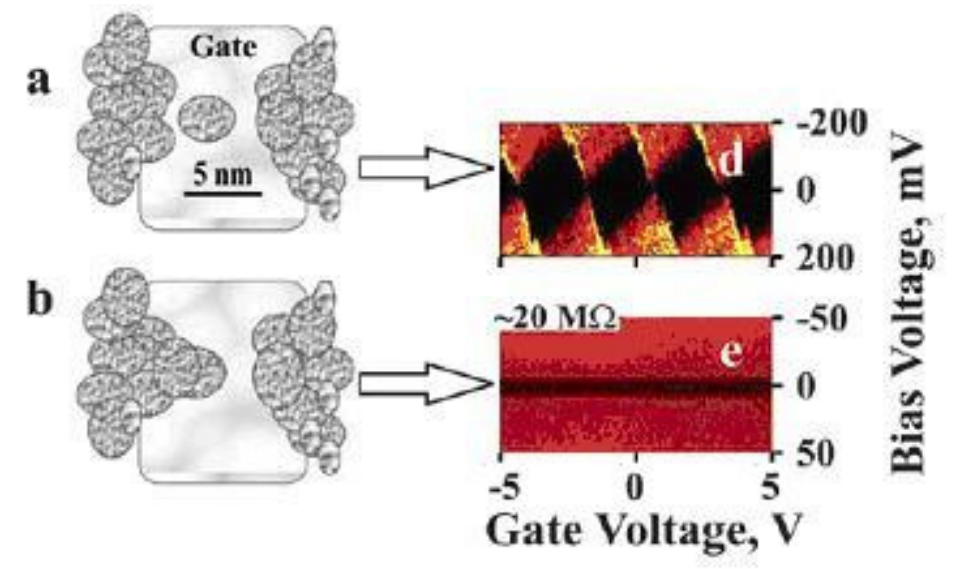

Figure 4.3 Some results from Ref. 54. (a) shows when a single gold grain is left between the source and drain electrodes, the corresponding differential conductance plot has Coulomb diamonds (shown in (d)); (b) when the grain is attached to one of the leads by changing the deposition conditions, the differential conductance does not depend on the gate voltage (shown in (e)).

\section{(2) More Complicated Results from Au Grains}

The gate-dependent transport measurements of Kondo impurities in bare gold break junctions has been reported in a study which showed that $30 \%$ of the broken devices have an enhanced zero-bias conductance. ${ }^{47}$ Figure 4.4(a) show Coulomb diamonds like that of Ref. 49. Figures 4.4(b) to (c) show a Kondo-like effect that in one case depends on the gate voltage and in the other that does not.

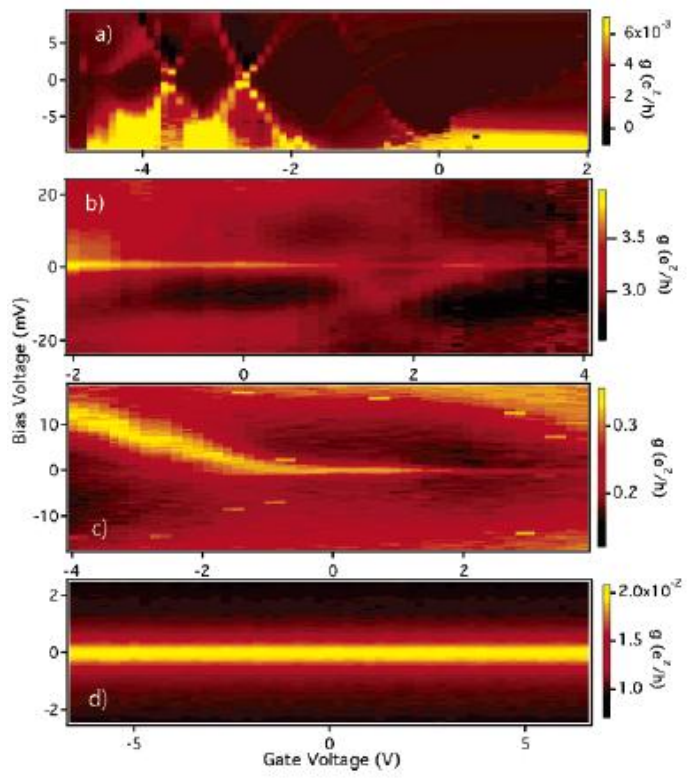

Figure 4.4 Differential conductance for four blank Au devices (at $250 \mathrm{mK}$ ) (from Ref. 47). (a) Coulomb blockade. (b) Superposition of a Coulomb blockade diamond and the Kondo effect. (c) Transition from Kondo effect to Coulomb diamond. (d) Kondo effect that is gate-independent. 


\section{(3) Two Quantum Dots (Simulation)}

Some papers have discussed the behavior of two quantum dots connected in series. ${ }^{56,57}$ In general, it is impossible to ensure that there is only one quantum dot in the gap of the electrodes. ${ }^{53}$ For example, although electron transport could be dominated by a single quantum dot, other dots around it could affect its transport behavior by capacitance coupling. ${ }^{53}$ Simulations of two metal grains in the gap of electrodes will be introduced below in order to distinguish transport mediated by two molecules from two metal grains in the next chapters.

Figure 4.5 shows an arbitrary double-drain system and the theoretical results concerning the multigrain systems. ${ }^{53}$ Figure 4.5 (a) shows the system topology and energy decrements for each tunneling jump. $\Delta K_{\alpha, \beta}$ is the electrostatic charging energy decrement during the tunneling jump of an electron between the grain $\alpha$ and the electrode $\beta$. Figure 4.5(b) shows the differential conductance graph as a function of bias and gate voltage for the general case of a double-dot SET. The top graph is the result of a Monte Carlo simulation and the bottom graph is the result of direct numeric integration. Figure 4.5(c) show all possible cases for a double-dot SET. 

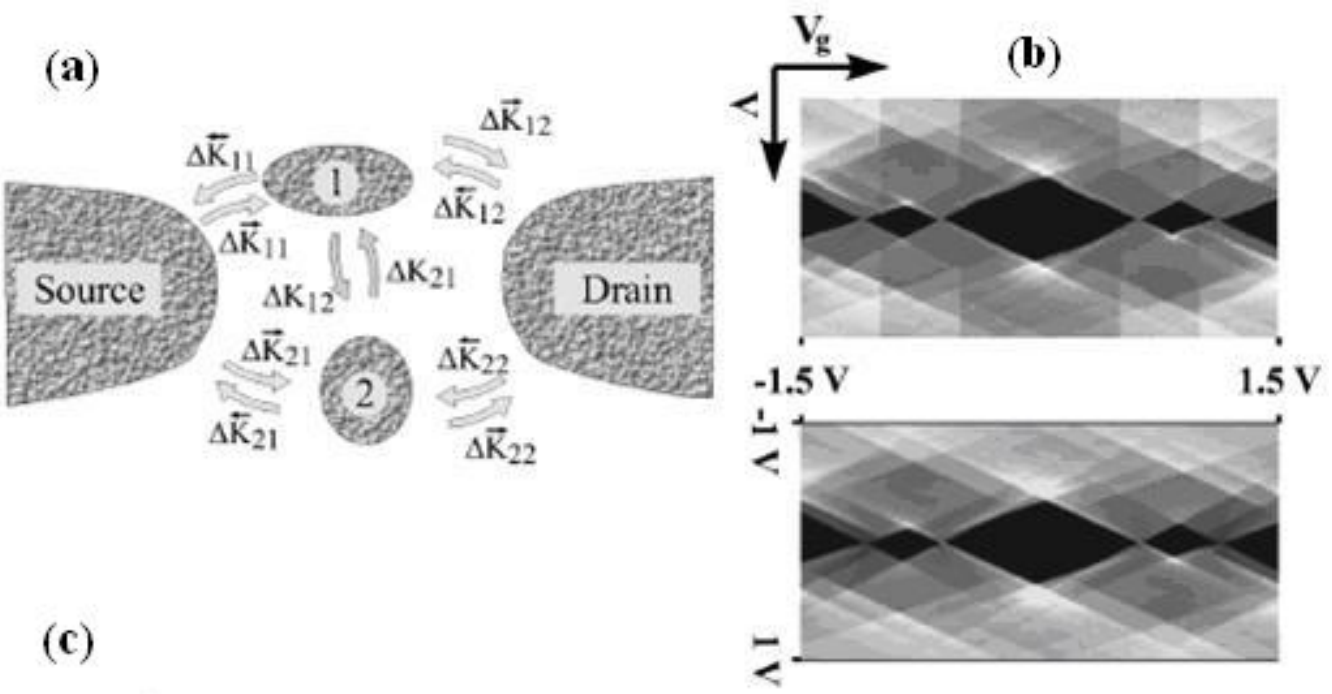

(c)

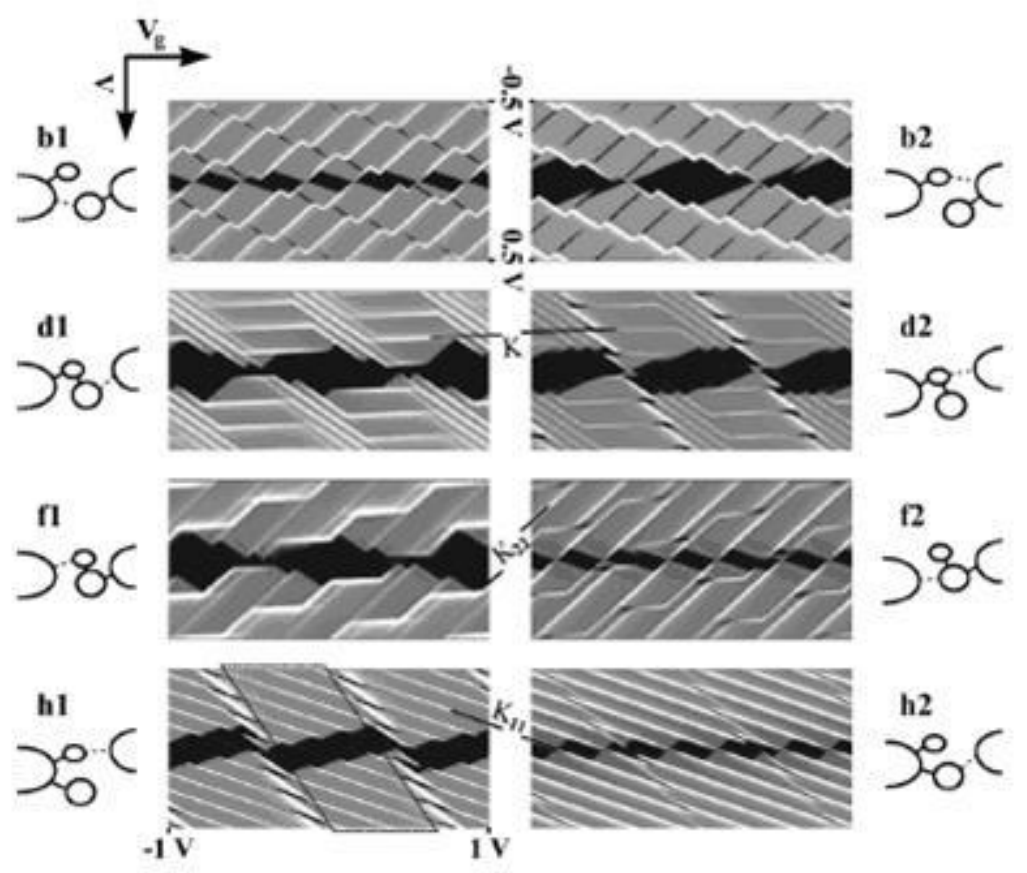

Figure 4.5 Simulation for two metal grains (from Ref. 53). (a) shows the system topology and energy decrements for each tunneling jump. (b) shows the differential conductance graph as a function of bias and gate voltage for the general case of a double-dot SET. The top graph is the result of a Monte Carlo simulation and the bottom graph is the result of direct numeric integration. (c) show all possible cases for a double-dot SET.

Another simulation has discussed negative differential conductance (NDC) from two quantum dots connected in series. ${ }^{58}$ The proposed theory is based on a diagrammatic technique for non-equilibrium many-body operator Green functions. It suggests that scattering between the states in the two quantum dots suppresses the total current greatly as the bias voltage is increased. The effect is present in Fig. 4.6 as the solid line for the 
system where the double-quantum-dot states are asymmetrically coupled to the electrodes. This theory predicts that there is no NDC for symmetric coupling.

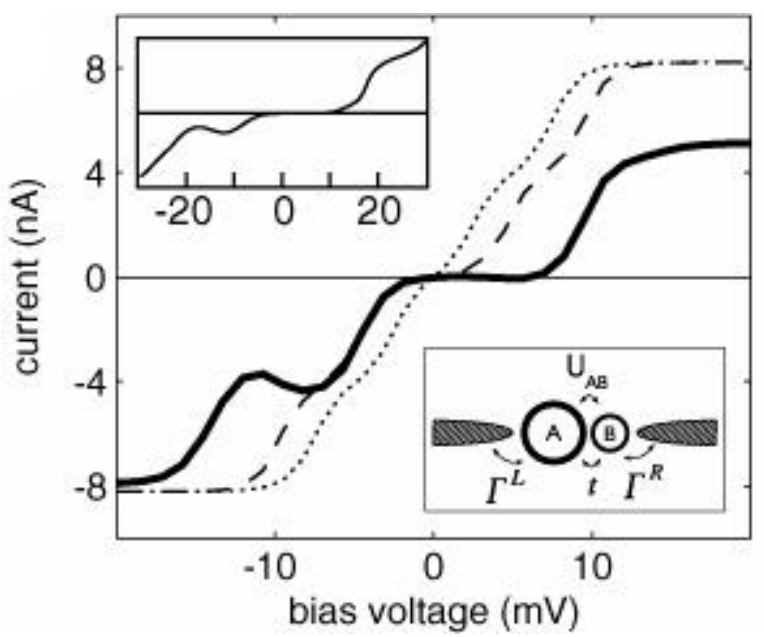

Figure 4.6 I- $V$ characteristics of the two quantum dots system (from Ref. 58). The solid line for the system where the double-quantum-dot states are asymmetrically coupled to the electrodes.

\subsection{Localized Heating}

The local temperature to which metal junctions become heated during the electromigration process is not well understood. Some groups have discussed this problem because the molecules used for making single-molecule transistors are often attached to the device before electromigration is performed, and high temperatures might damage or destroy some molecules. ${ }^{59,60,61}$ This issue is especially important for biomolecules which can be easily denatured at high temperatures (above room temperature). One estimate of the temperature of a gold junction during EM is that it is a few hundred Kelvins, low enough to allow the study of many molecular adsorbates without desorption or dissociation of the molecules. ${ }^{59}$ Another estimate for the effective temperature experienced by molecular adsorbates on the gold junction during the electromigration process is $515 \mathrm{~K}$ at least. ${ }^{60}$

The temperatures reached during electromigration in Pt junctions are considerably higher than those observed for Au junctions. ${ }^{61}$ The heating in Pt junctions has been investigated before and during electromigration using thermal emission microscopy. The wires were observed to reach temperatures in excess of $1000 \mathrm{~K}$, which was measured in air at room temperature. ${ }^{61}$ The effective temperature during the electromigration process 
experienced by molecular adsorbates on a metal junction cooled down to cryogenic temperatures is not known.

\subsection{Some Effective Methods}

Considering the shortcomings of the break-junction method discussed above, improvements can be made. One method is breaking the junction first and then depositing molecules. After that, the devices are cooled down and measured. This method has been successfully used to study discrete energy-level spectra in individual chemically synthesized gold nanoparticles. ${ }^{62}$ Some previous papers have discussed to make similar devices to trap metal nanoparticles to study Coulomb blockade. ${ }^{63,64}$

Another method for measuring bio-molecules is the self-breaking junction. The mechanism of this method is not well understood. A thin film with thickness less than 10 nm will become discontinuous when cooling down to cryogenic temperatures. ${ }^{13}$ The thickness in the middle of the junction can be made to be less than $10 \mathrm{~nm}$ by using magnetron sputtering with approximately a $15^{\circ}$ degree deposition angle with respect to the surface normal of the sample. In this case, the junction will demonstrate some cracking from the middle at low temperature (shown in Figure 4.7(A)), probably due to surface stress. The metal thin film does not recover when warmed up to room temperature. Another possibility is that the junction is broken already during fabrication or loading. This result is different with from what happens after breaking by an electrostatic discharge (shown in Figure 4.7(B)). Whether small metal grains are formed in the gap region need to be determined. At least, using self-break method can avoid the problem of the local heating during the electromigration process. 


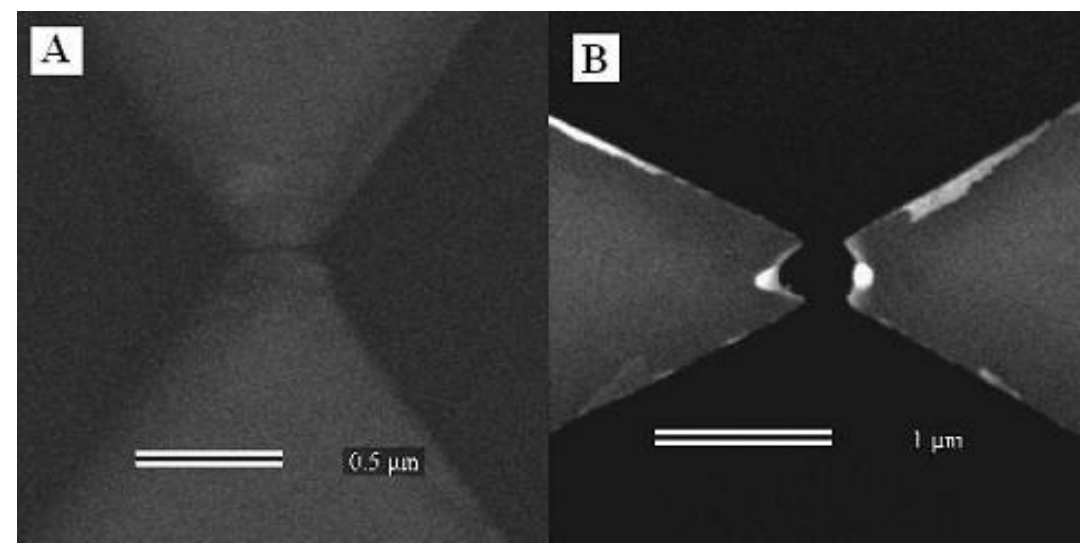

Figure 4.7 Two broken junctions. (A) Self-break. (B)Break by electrostatic discharge.

\subsection{Summary}

In this chapter, electron transport by metal grains ( $\mathrm{Pt}, \mathrm{Au})$ was discussed. One important distinction between transport through single molecules and metal grains is the characteristic of electron-molecular vibration coupling. The discrete vibrational modes of the molecules (as opposed to continuous levels expected for metallic islands) have been observed in several experiments.

The technique of electromigration has the disadvantage that small gold grains are formed in the gap region. Unfortunately, a more reliable method suitable for measurement protein conductance does not exist at the present time. 


\section{Chapter 5}

\section{Protein Immobilization}

\subsection{Introduction}

There has been a growing interest in immobilizing proteins on a surface in order to study the electron transfer mechanism in a single protein. A major concern regarding protein immobilization is whether they can retain their functionality. Therefore, protein immobilization on different chips has become a broad research area and some methods have been successful. ${ }^{65,66}$

Protein immobilization techniques can be classified into three different mechanism based on how the molecule is bound to the carrier, either by covalent binding, adsorption or bioaffinity immobilization. A few of these techniques will be introduced in this chapter and then the effects of these methods on the electrical measurements will be discussed.

\subsection{Covalent Binding}

Covalent binding is the most widely used technique for protein immobilization. This method is based on the development of covalent bonds between the protein and the support. Covalent linkages are strong and the protein will not be released from the support material easily. Some proteins can covalently bind to the immobilization support by its functional groups of exposed amino acids. The covalent bond formed between the protein and the substrate should involve amino acid residues located away from the active site of the protein in order to maintain protein biological activity.

Covalent binding requires the activation of specific functional groups of the support. It is also possible to introduce into the protein being immobilized additional reactive groups by chemical modification. These chemical modifications of either the protein or support are performed to obtain a strong and specific attachment of the protein to the support.

\subsubsection{DTSP}


For proteins with cysteines on the surface, direct bonding to Au or Pt substrates is possible. Myoglobin has no cysteines and an alternative approach is required. Here, the linker DTSP (dithiobis [succinimidyl propionate], SIGMA) was used (shown in Fig. 5.1). ${ }^{67}$ This linker bonds to the Pt surface via a thiol group. ${ }^{68}$ The succinimidyl portion of the linker can be bonded to the protein at either the N-terminus or via a surface lysine. The thiol linker, which is than $1 \mathrm{~nm}$ in length, was used in some of our experiments with myoglobin. In these cases, the DTSP was dissolved in DMF ( $N, N$-dimethylformamide, Sigma) and the Pt chip immersed in this solution for at least 24 hours. The Pt chip was then washed with PBS and immersed in a PBS solution of the myoglobin for 24 hours. The Pt chip was then removed and washed with water. This procedure resulted in the myoglobin being bonded to the Pt chip via a DTSP linker (shown in Fig. 5.2).

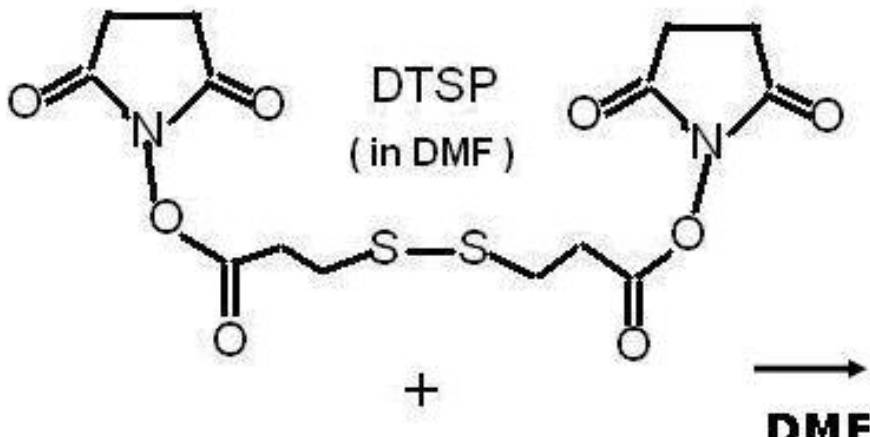

All or Pt

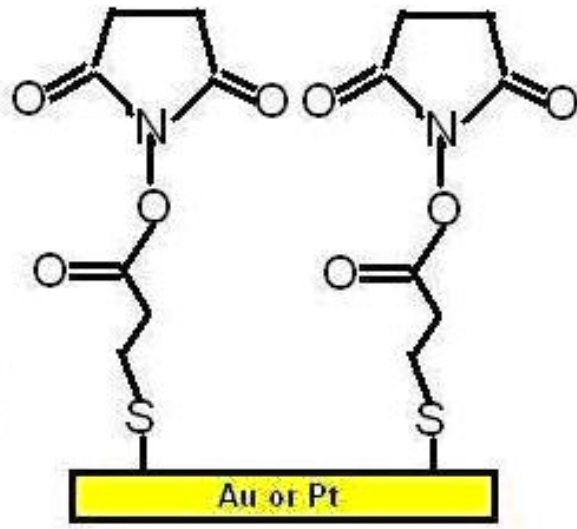

Figure 5.1 DTSP can bind with Au or Pt in DMF.

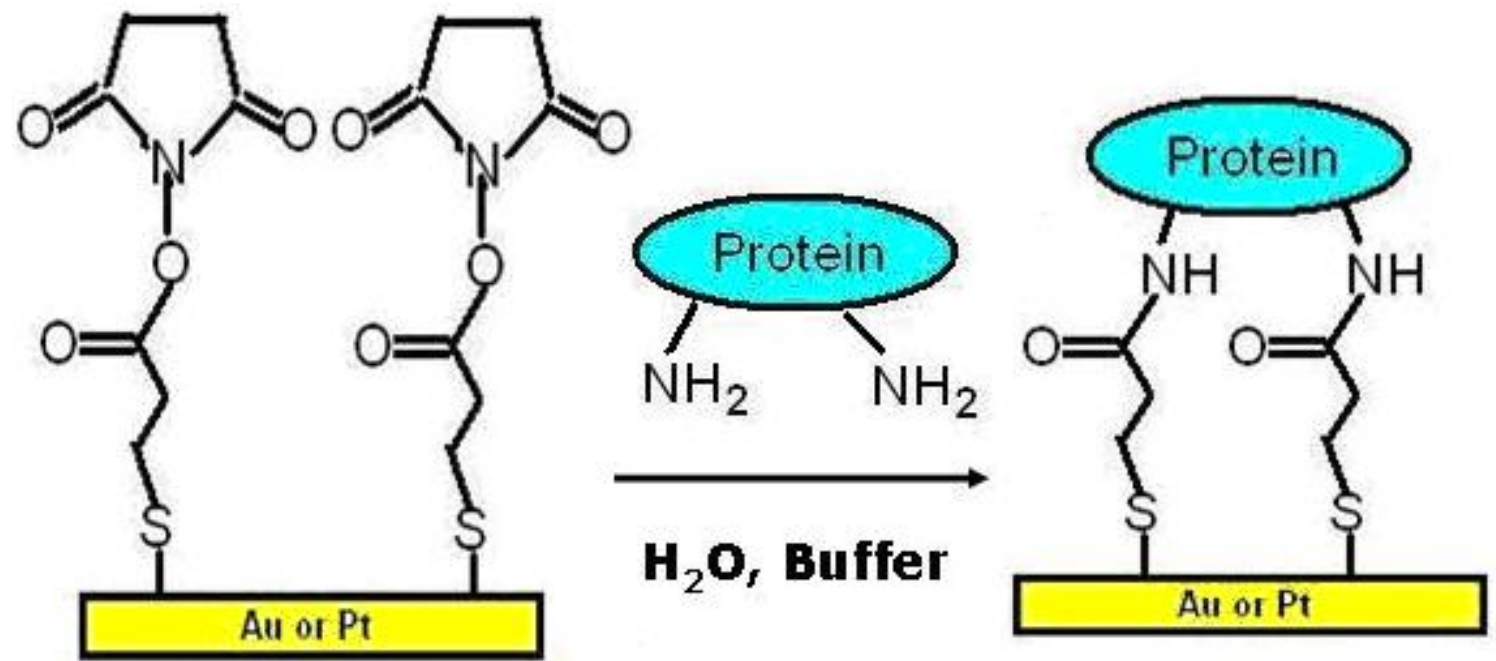

Figure 5.2 DTSP used to bind a protein to a Au or Pt surface. 
At total of $53 \mathrm{Pt}$ junctions coated with DTSP were broken with feedback by electromigration at $77 \mathrm{~K}$. Out of these, 12 junctions showed conductance peaks. Figure 5.3 show two samples bound with DTSP from the 12 samples. Because the linker itself has a significant electronic signature, the data analysis of proteins where these types of linkers are used will be more complicated (Fig. 5.4).
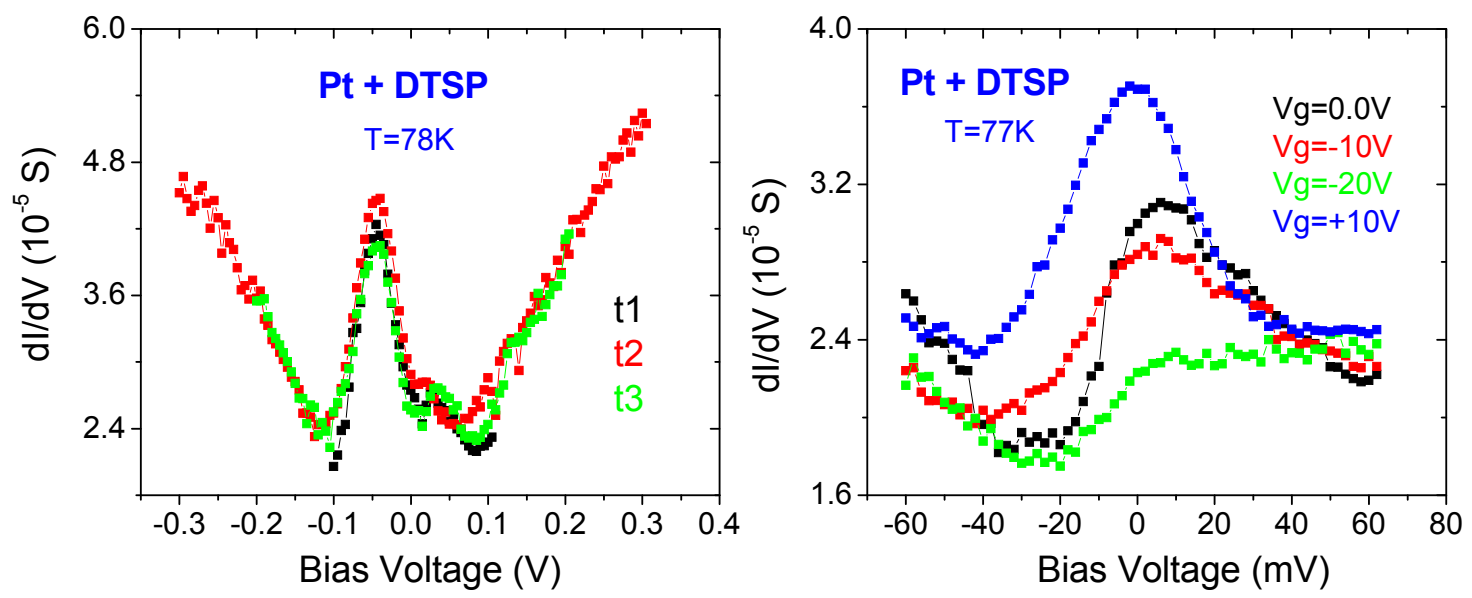

Figure 5.3 Two results of DTSP at $77 \mathrm{~K}$. Left graph shows there are two reproducible conductance peaks, measured at different times: $\mathrm{t} 1, \mathrm{t} 2, \mathrm{t} 3$. Right graph shows there is one conductance peak dependence of gate voltage.
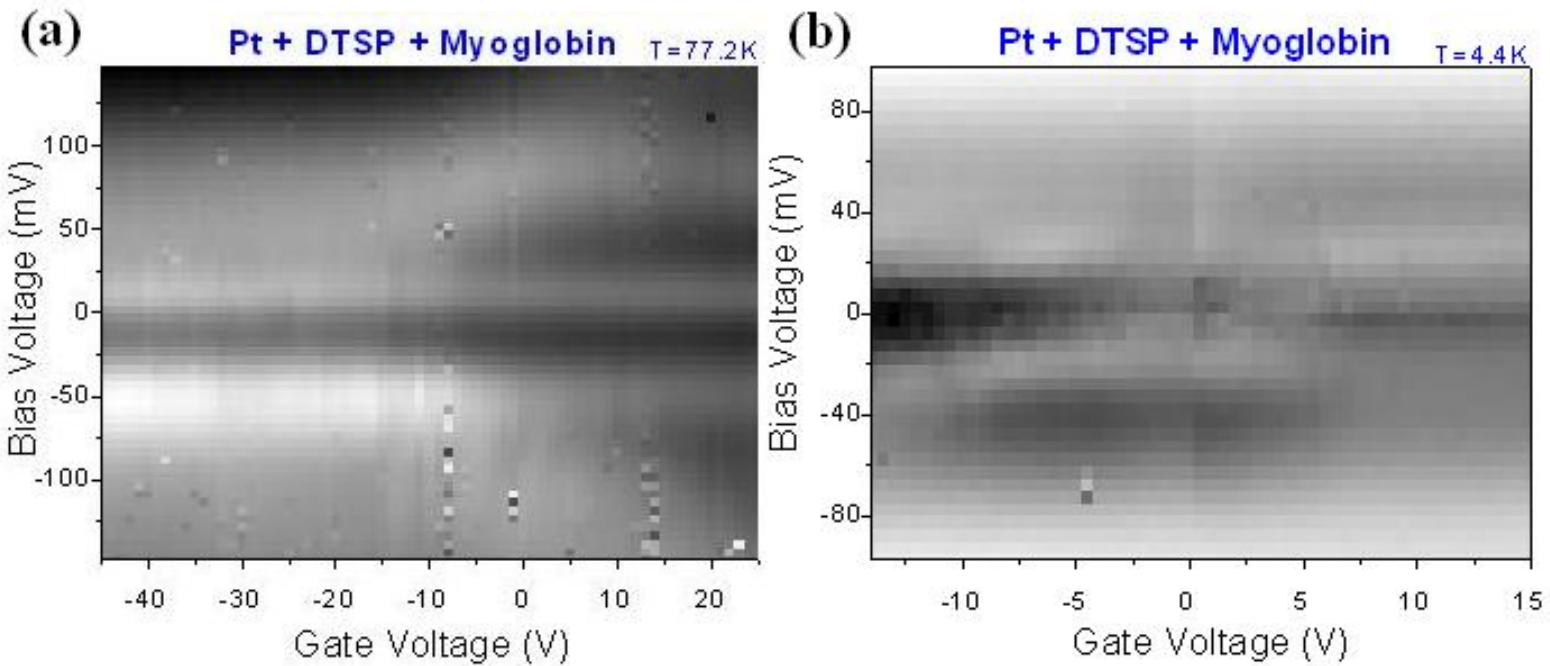

Figure 5.4 Two results of myoglobin bound with DTSP. (a) One result at $77 \mathrm{~K}$. (b) One result at 4 K. 


\subsubsection{Cystamine}

Cystamine, which is another kind of linker, had the same problem as DTSP. In our experiments, cystamine dihtdrochloride (98\%) was used as linker. Cystamine can be dissolved in DI water and can be bonded on a Pt junction by the thiol group as shown in Fig. 5.5. Fourteen Pt junctions coated with cystamine were broken by electromigration at $77 \mathrm{~K}$ with feedback. Three junctions showed one conductance peak. Figure 5.6 shows two results from cystamine. One peak can be found in each sample. The peak is not stable and may be due to, for example, the molecule being only bound to one side of the gap junction.

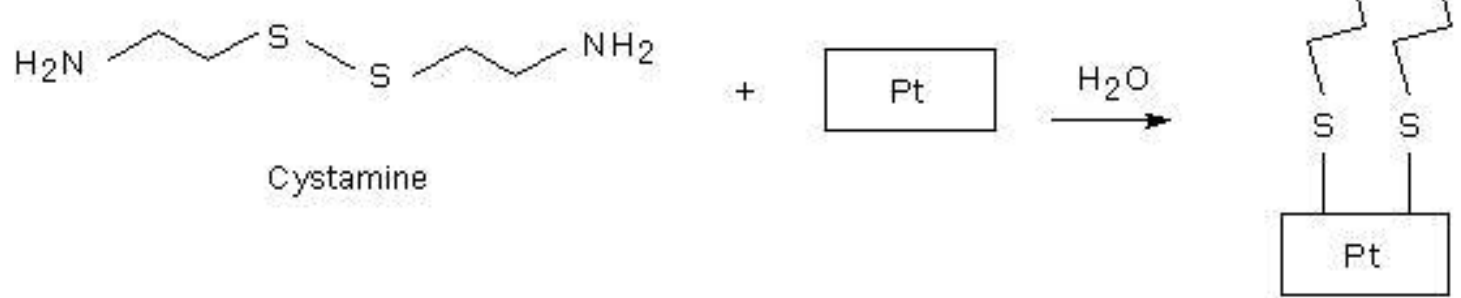

Figure 5.5 Cystamine can bind with Au or Pt in $\mathrm{H}_{2} \mathrm{O}$.

(a)

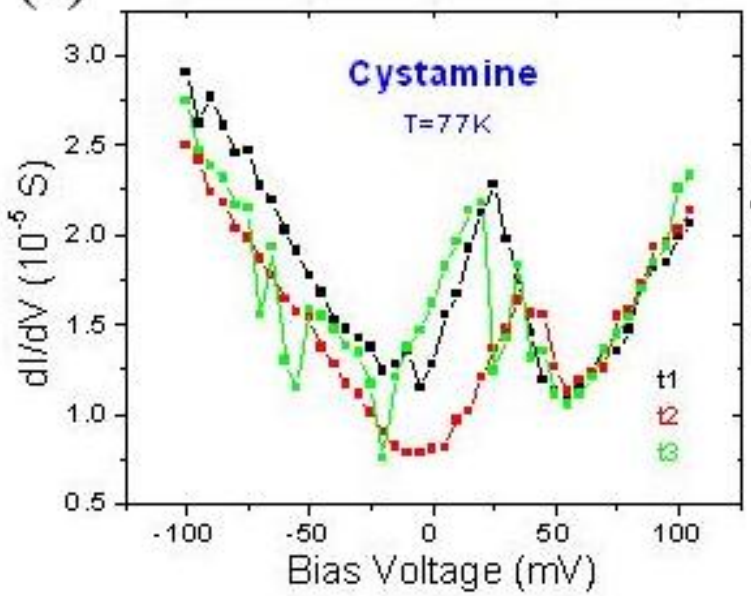

(b)

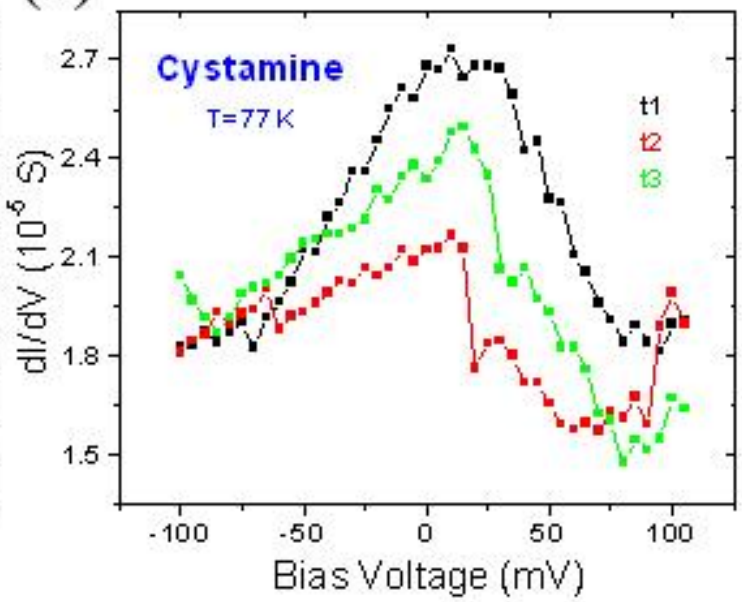

Figure 5.6 Two results from Cystamine bound with Pt at $77 \mathrm{~K}$. (a) One sample with one conductance peak at $77 \mathrm{~K}$. (b) Another sample at $77 \mathrm{~K}$ measured at different time: t1, t2, t3.

In summary, two popular linkers used to bind organic molecules and biomolecules to $\mathrm{Au}$ and other noble metals are DTSP and cystamine. Both of these 
molecules rely on a thiol group to bind to the noble metal and an amine group on the opposing end to bind to the protein. These kind of linkers may provide additional tunneling channels for electron transport, thus complicating the data analysis. Therefore, we elected not to use a linker to attach the proteins to our devices.

\subsection{Physical Immobilization}

Proteins can be adsorbed on the surface of a substrate by intermolecular forces. The forces responsible for this kind of immobilization are relatively weak compared with covalent binding. They include hydrogen bonds, van de Waals forces, and hydrophobic interactions. $^{69}$ In addition, adsorption may involve ionic binding, based on ionic interactions between the protein and the opposite charge of the carrier. The weakness of the forces involved in protein immobilization could allow desorption of the protein from the support. Changes in $\mathrm{pH}$, ionic strength, and temperature disrupt the interactions between the protein and the carrier. Proteins immobilized by adsorption, however, are expected to retain high activities. ${ }^{70}$ Adsorption mainly occurs with three-dimensional porous materials, ${ }^{66}$ but in our experiments, direct deposition was achieved as in experiments performed on smaller molecules. ${ }^{71}$

In our experiments, a $0.01 \mathrm{M}$ phosphate buffered saline solution (PBS) (Sigma) $\left(\mathrm{NaCl}-0.138 \mathrm{M}\right.$; KCl-0.0027M, pH 7.4 at $25{ }^{\circ} \mathrm{C}$ ) was used to prevent denaturing of the protein. The protein used here was essentially salt-free. As a reference, myoglobin without PBS was also measured. In the latter case, the conductance peaks were not stable (i.e., it was difficult to reproduce the same conductance as a function of bias voltage for different runs on the same sample, as shown in Fig. 5.7). This could be a result of protein conformation changes induced by the applied electric field. Because some amino acids have positively charged groups and some have negatively charged groups, differently charged functional groups may react differently to an applied electric field, thereby causing conformation changes in the protein. One possible reason that PBS could stabilize the protein is that the negatively charged groups of amino acids attract the $\mathrm{Na}^{+}$ and $\mathrm{K}^{+}$ions in the PBS solution and the positively charged groups of amino acids attract the $\mathrm{Cl}^{-}$ions. The other reason is that PBS may crystallize while drying, trapping the protein in place. 


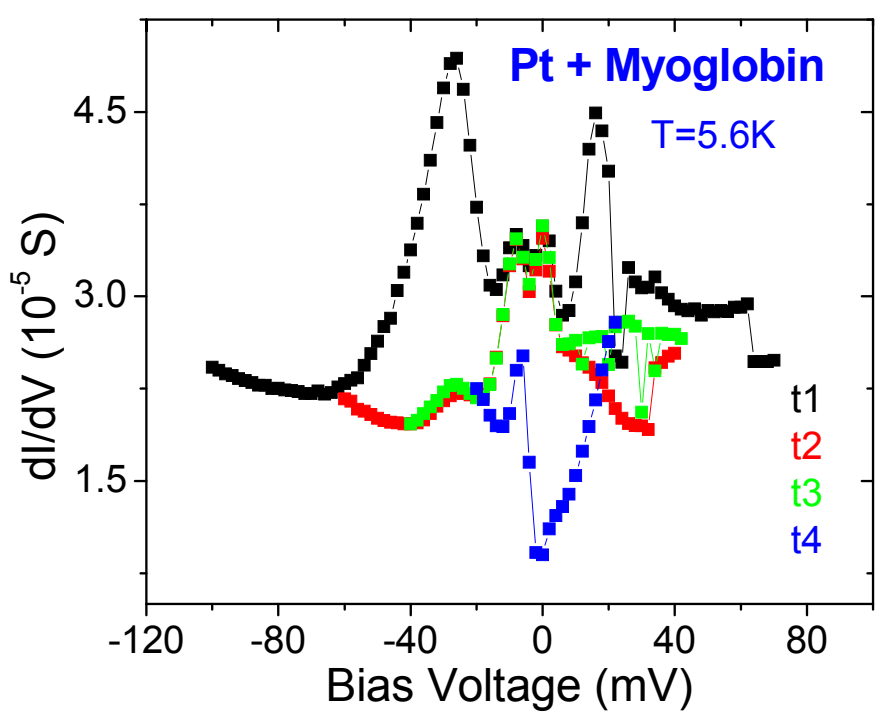

Figure 5.7 One myoglobin sample without PBS measured at different time: $\mathrm{t} 1, \mathrm{t} 2, \mathrm{t} 3$ and $\mathrm{t} 4(\mathrm{t} 1<\mathrm{t} 2<\mathrm{t} 3<\mathrm{t} 4)$.

\subsection{PBS Control Experiments}

Conductance measurements with only PBS (no protein) in 50 junctions were performed at $77 \mathrm{~K}$ and three junctions at $5.5 \mathrm{~K}$. Figure 5.8(a) shows the conductance of one sample after breaking the junction at $77 \mathrm{~K}$. Only one of these samples had a conductance step or peak at $77 \mathrm{~K}$, perhaps as a result of an impurity in that sample (shown in Fig. 5.8(b)). The mechanism of electrical conductivity in alkali halide crystals is usually the motion of ions and not the motion of electrons ${ }^{37} ; \mathrm{NaCl}$ and $\mathrm{KCl}$ are insulators with an energy band gap of over $8 \mathrm{eV}$, and therefore tunneling is difficult via these energy levels. 
(a)

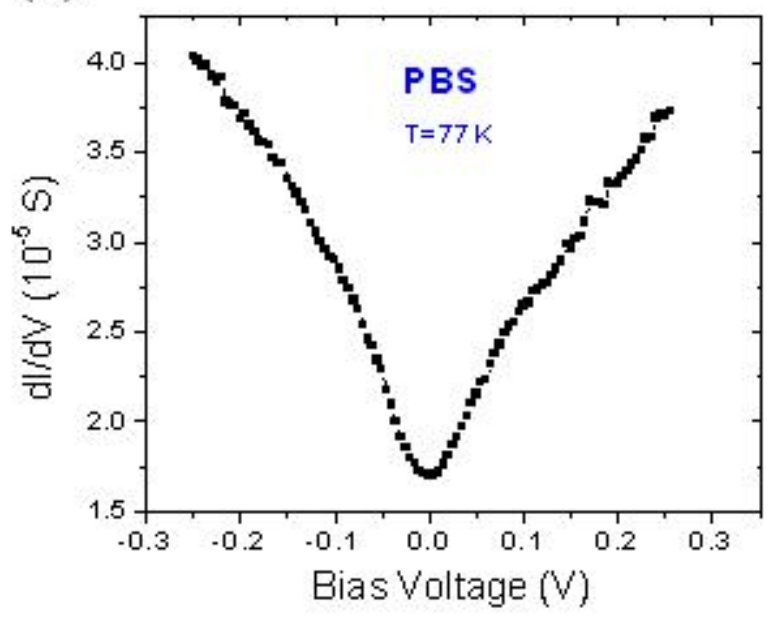

(b)

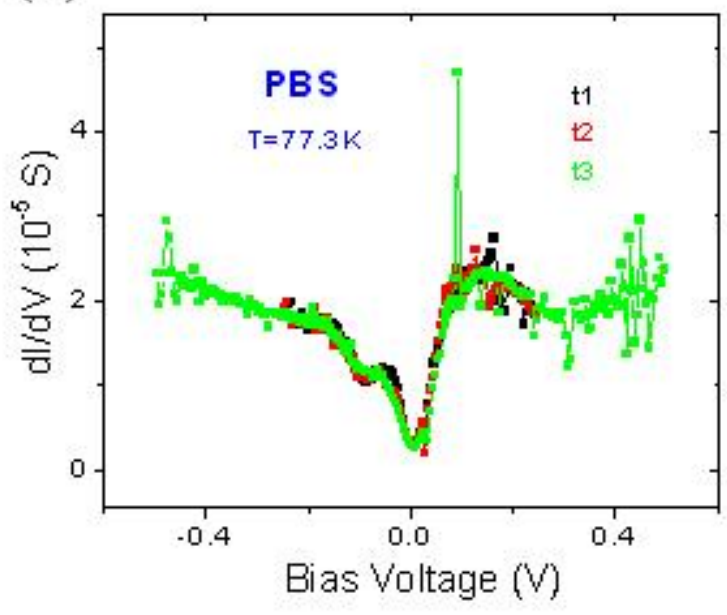

Figure 5.8 Two results from PBS at $77 \mathrm{~K}$. (a) One sample without conductance peak at $77 \mathrm{~K}$. (b) One sample had a conductance step or peak at $77 \mathrm{~K}$, Measured at different time: $\mathrm{t} 1, \mathrm{t} 2, \mathrm{t}$.

\subsection{Summary}

Several strategies were developed to immobilize protein on a chip in protein biochip technology. While it is difficult to tell which strategy is the best one, it is important to avoid the interference from the background signals. 


\section{Chapter 6}

\section{Protein Vibrational Spectroscopy}

\subsection{Introduction}

As discussed in the Chapter 2, a molecular vibration is excited when the molecule absorbs a quantum of energy $\hbar \omega$, corresponding to the vibration's resonant frequency $\omega$. The vibrational states of a molecule can be probed with infrared and Raman spectroscopies. These two methods are complementary because some vibrational transitions that are observed in IR spectroscopy are not observed in Raman spectroscopy, and vice-versa.

The vibrational spectra of proteins are complicated and are determined by its structure and function. Some information can be derived from the vibrational spectra, such as the redox state, chemical structure, bond lengths and bond strength, bond angles and conformation, hydrogen bonding, etc.

In this chapter, vibrations will be discussed first in terms of a simple diatomic oscillator in order to illustrate some of the fundamental principles that govern the relationship between the vibrational spectrum of a molecule and its structure and environment. Then, Raman spectroscopy will be introduced. After that, Raman results of apomyoglobin and myoglobin deposited on Pt and cooled to cryogenic temperatures will be discussed.

\subsection{Vibrational State}

The vibration of a diatomic molecule can be regarded as if the two atoms were joined by a spring, and stretching and contracting of the spring results in the vibrations. In this model, the chemical binding force in the diatomic molecule behaves like a spring. ${ }^{72,73}$ 


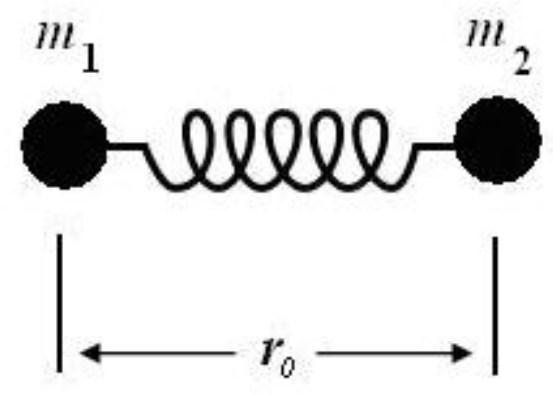

Fig. 6.1 Equivalent diatomic molecule.

For a diatomic molecule, consisting of two atoms with masses $m_{1}$ and $m_{2}$, the Schrödinger equation can be written in their center of mass coordinate as

$$
\frac{\hbar^{2}}{2 m} \nabla^{2} \psi+[E-V(r)] \psi=0
$$

Here $m=m_{1} m_{2} /\left(m_{1}+m_{2}\right)$ is the reduced mass and $r$ is the distance between the two nuclei. To find the vibrational energy levels, the form of $V(r)$ must be known. In the simplest case, we can take $V(r)$ to be parabolic,

$$
V(r)=\frac{1}{2} k\left(r-r_{0}\right)^{2}
$$

where $k$ is a force constant between the two atoms and $r_{0}$ is the equilibrium distance. With this potential, the solution of the Schrodinger equation for the energy of the vibrational mode in one dimension is

$$
E_{v}=\left(n+\frac{1}{2}\right) \hbar \omega_{0},
$$

where $\omega_{0}$ is the classical vibration frequency, $\omega_{0}=\sqrt{k / m}$, and $n$ is an integer greater than or equal to zero. The resonant frequency increases as the force constant increases, that is, when the electron density in the bond between the two atoms increases. Any inter- or intramolecular factor that alters the electron density in the bonds will affect the vibrational spectrum. The second important influence on the frequency is the mass of vibrating atoms; the larger the mass, the slower the vibration. This effect is often used as a tool for interpreting spectra when the sample is isotopically labeled in order to shift the frequency of vibrations that involve the labeled atoms. 
Figure 6.2 show the vibrational energy levels, vibrational-zero-point energy and dissociation energy of a diatomic molecule. The transitions among these vibrational levels follow the selection rule $\Delta n= \pm 1$, where $\Delta n=+1$ corresponds to absorption and $\Delta n=-1$ corresponds to emission. ${ }^{73}$

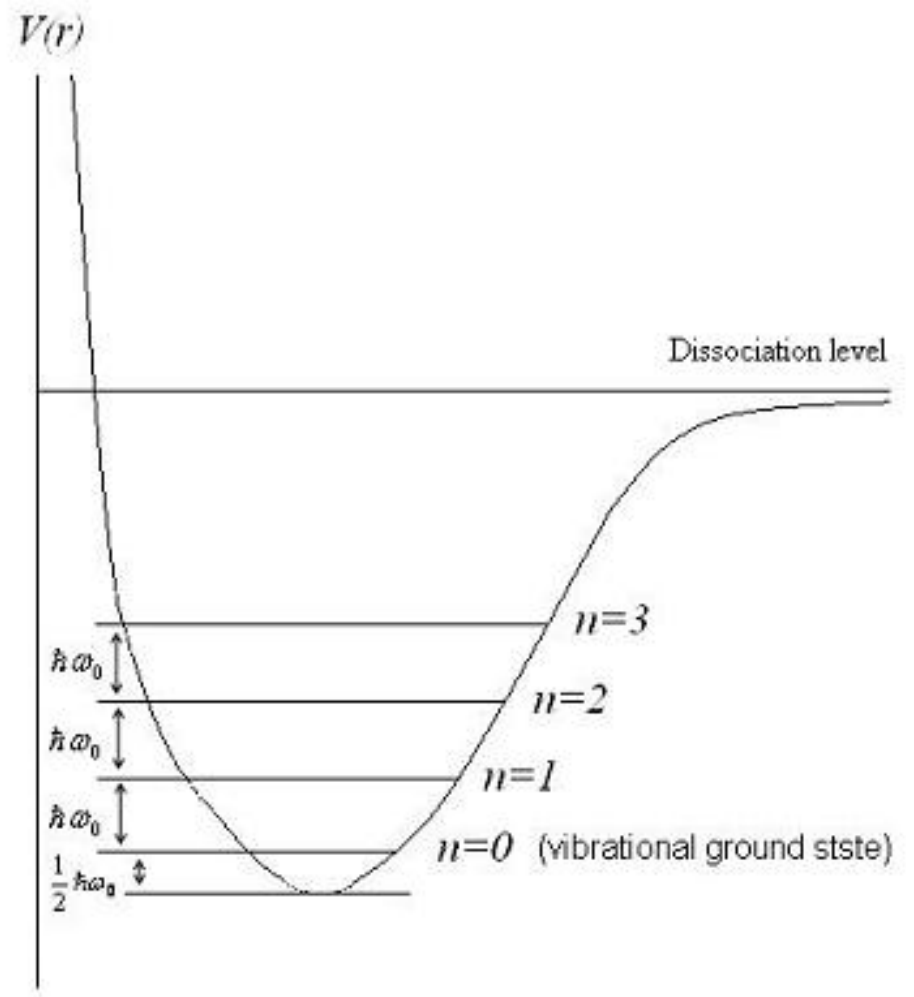

Figure 6.2 The vibrational levels of a diatomic molecule.

Raman spectroscopy can be used to identify chemical compounds because the frequencies of vibrational transitions depend on the atomic masses and the bond strengths. Heavier atoms correspond to lower vibrational frequencies, while stronger bonds correspond to higher vibrational frequencies. Thus, armed with a database of spectra from known compounds, one can unambiguously identify many different known chemical compounds based on a Raman spectrum. The number of vibrational modes scales with the number of atoms in a molecule, which means that the Raman spectra from large molecules will be very complicated. For example, proteins typically contain thousands of atoms, and will therefore have thousands of vibrational modes. 


\subsection{Raman Spectroscopy}

Raman spectroscopy relies on inelastic scattering of light usually provided by a laser in the visible, near infrared, or near ultraviolet range. The laser light interacts with phonons or other excitations in the system, resulting in the energy of the laser photons being shifted up or down. The shift in energy gives information about the phonon modes in the system.

The Raman effect occurs when light excites a molecule and interacts with the electron cloud of the bonds of that molecule. The incident photon excites one of the electrons into a virtual state. For the spontaneous Raman effect, the molecule will be excited from the ground state to a virtual energy state, and relax into a vibrational excited state, which generates Stokes Raman scattering. If the molecule was in an excited vibrational energy state to start with, the energy can be absorbed by the incoming photon, thus generating anti-Stokes Raman scattering (showed in Fig. 6.3). It is important to note that the intensity of the anti-Stokes lines will vanish while the relative intensity of the Stokes lines will increase as $T \rightarrow 0 .^{37}$ This is because as the temperature is lowered, the probability of the molecule being in an excited state due to temperature is diminished, while absorption of energy from the incoming photon is always possible.

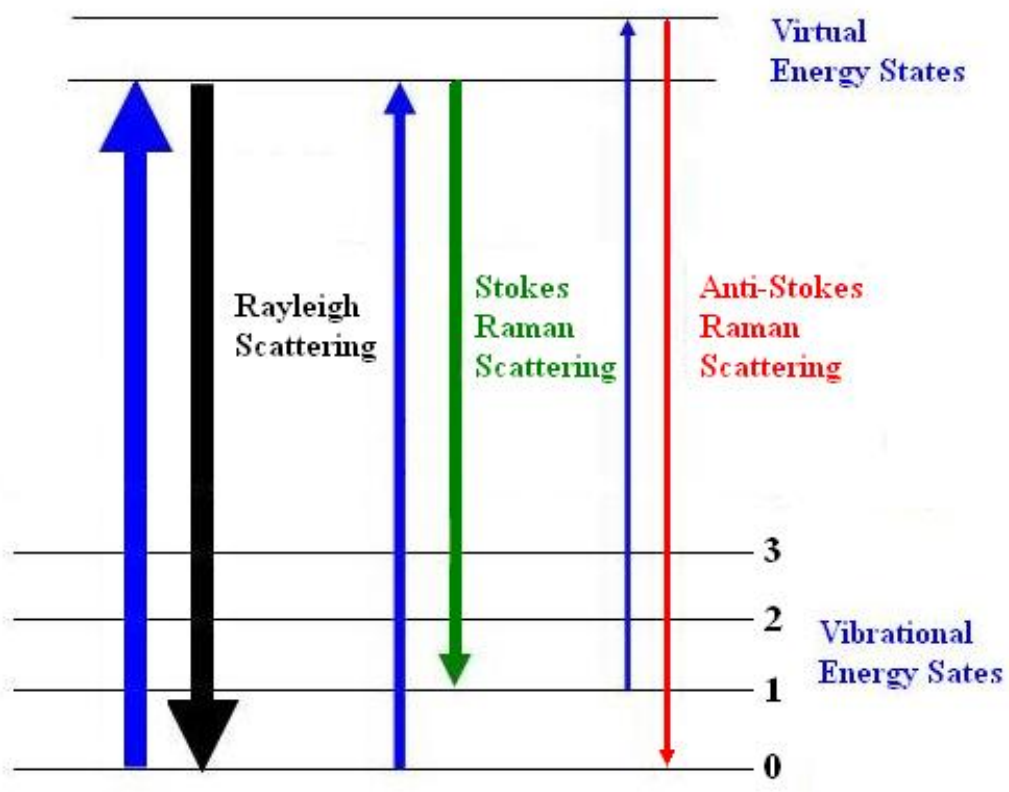

Figure 6.3 Raman energy levels. 
In resonance Raman spectroscopy, the energy of the incoming or scattered light is adjusted such that it coincides with an electronic transition of the molecule or crystal. In most materials the incoming and outgoing electronic resonances are sufficiently broad that they can not be distinguished. So, rather than exciting the molecule to a virtual energy state, it is excited to near one of its excited electronic transitions (showed in Fig. 6.4). When the frequency of the laser beam is tuned to be near an electronic transition (resonance), the vibrational modes associated with that particular transition exhibit a greatly increased Raman scattering intensity.

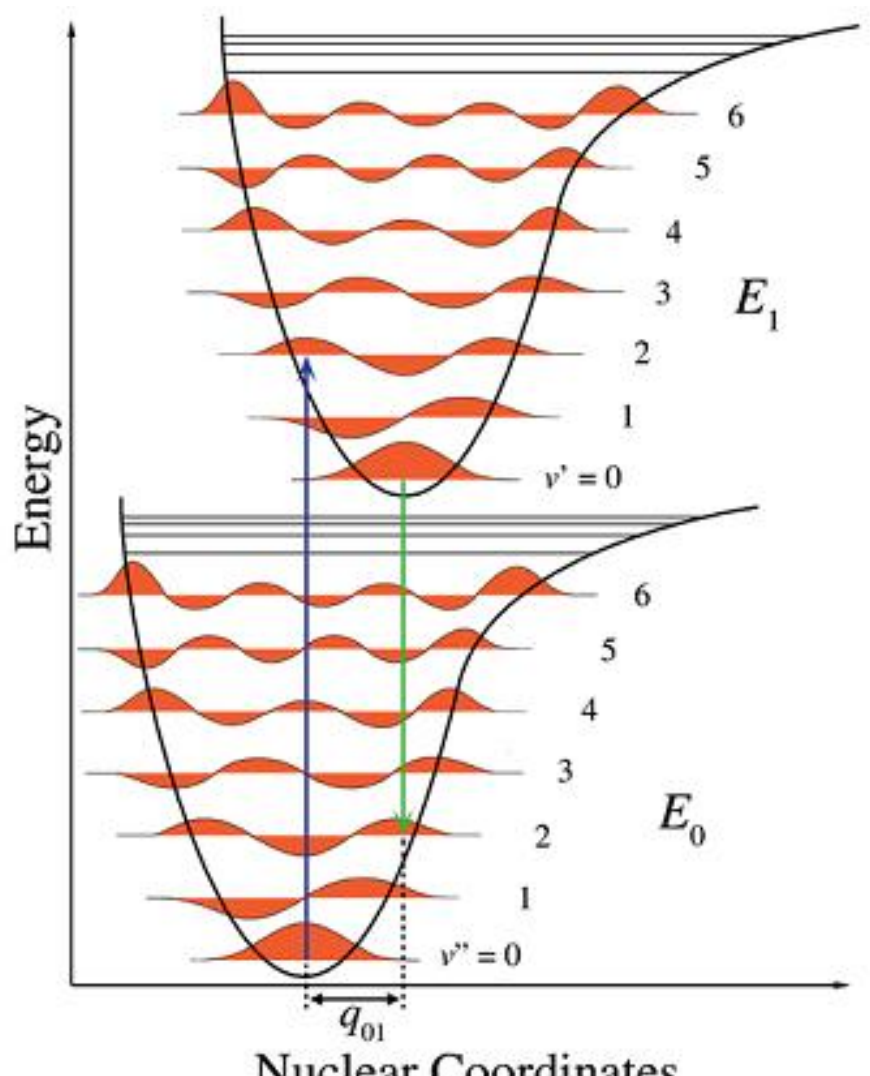

Figure 6.4 Vibronic transitions diagram based on the Franck-Condon principle (from Wikipedia).

\subsection{Raman Spectra for Myoglobin Proteins}

Raman spectra show that the heme group of myoglobin has two different electronic transitions which give rise to two bands near $400 \mathrm{~nm}$ (also known as Soret or 
$\gamma$ bands) and 500-550 $\mathrm{nm}$ ( $\alpha-\beta$ band) via different resonance scattering mechanisms. ${ }^{74}$ Recently, Raman spectra ( $\alpha-\beta$ bands with the $514.5 \mathrm{~nm}$ excitation and Soret band with $406.7 \mathrm{~nm}$ excitation) was obtained for a dry myoglobin film (Fig. 6.5). ${ }^{75}$

(a)

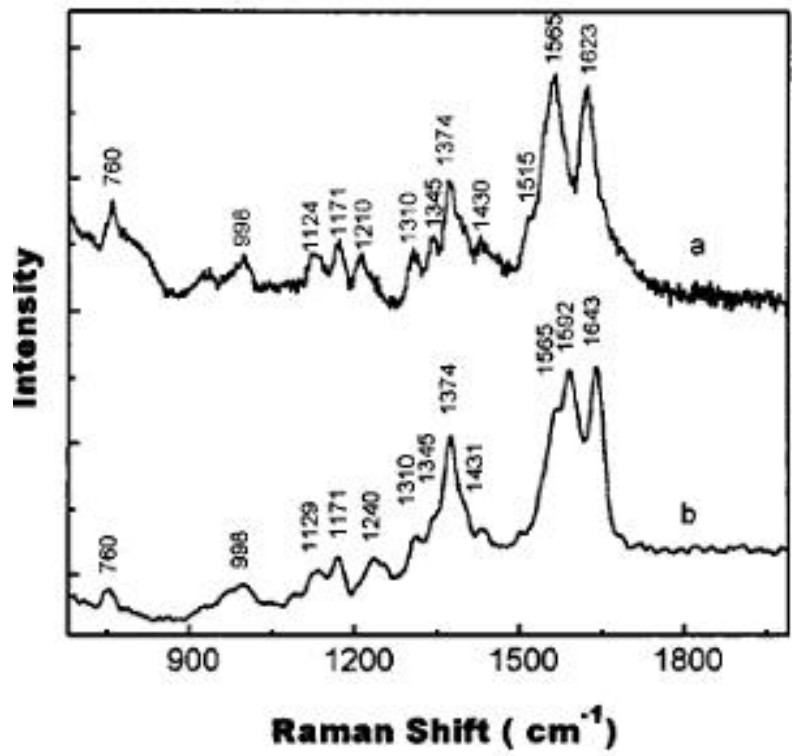

(b)

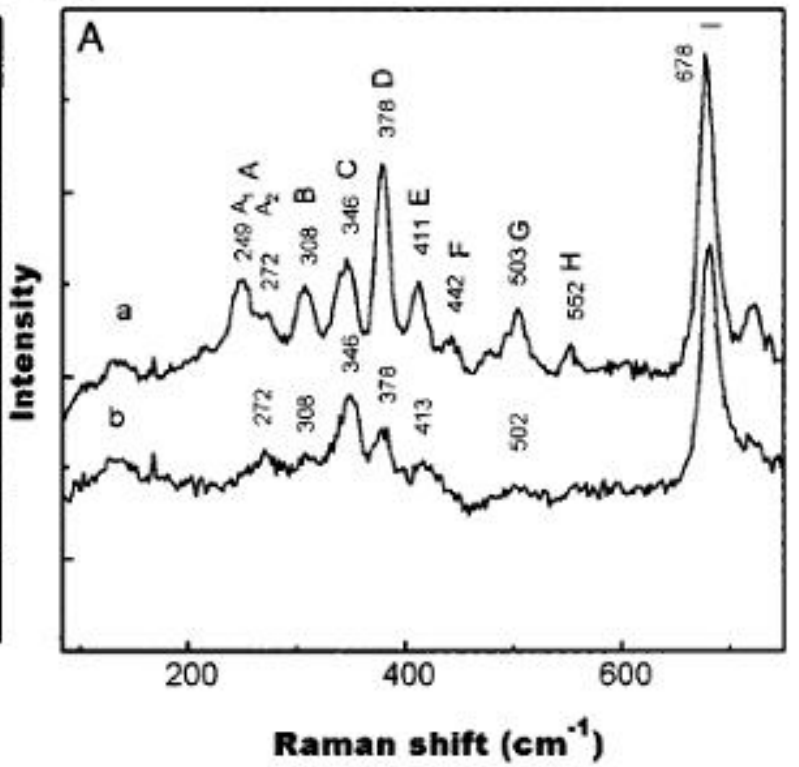

Figure 6.5 Resonance Raman spectra for aqueous metMb a and Mb film b in (a) $\lambda_{e x}=514.5 \mathrm{~nm}$ and (b) $\lambda_{e x}=406.7 \mathrm{~nm}$ (from Ref. 75$)$.

In order to perform measurements at cryogenic temperatures, it is important to determine whether the protein remains active. Raman specta ( $\alpha-\beta$ bands with the $514 \mathrm{~nm}$ excitation laser) performed on our samples show that it is still active after cooling down to $5 \mathrm{~K}$ for two days and then warming up to room temperature. Because we were able to observe these lines before and after cooling, it is unlikely that the protein denatured during the cooling process. The Raman vibration bands of apomyoglobin were also identical before and after cooling down to $5 \mathrm{~K}$ and then warming up to room temperature. These results are discussed in more detail below.

\subsubsection{Experimental Procedures}

The myoglobin and apomyoglobin investigated here were obtained from horse skeletal muscle (Sigma) in its powder state and essentially salt-free. The purity of myoglobin was at least $95 \%$ according to the manufacturer. The protein powder was dissolved in a $0.01 \mathrm{~mol} / \mathrm{L}$ PBS (Sigma, $\mathrm{pH}=7.4$ ) solution. The myoglobin and 
apomyoglobin concentrations were $1.42 \times 10^{-2} \mathrm{~mol} / \mathrm{L} 3.15 \times 10^{-5} \mathrm{~mol} / \mathrm{L}$, respectively. All protein solutions were filtered through the filters with $0.2 \mu \mathrm{m}$ pores (Whatman) to remove larger protein clusters that may have formed. Samples were prepared by placing a drop of the solution on a Pt thin film grown on Si substrates identical to those used for the break junction experiments.

A Renishaw Raman microscope was used to obtain the Raman spectra. The laser power was adjusted to $250 \mathrm{~mW}$ for $514 \mathrm{~nm}$ excitation to check the background. The spectrum was recorded with 30 seconds accumulation 15 times. Figure 6.4 shows the results of the background measurement. Figures 6.6(a) and 6.6(b) show the results from substrate. The sharp peak is from the silicon wafer. Figures 6.6(c) and 6.6(d) show the results from dry PBS. The results show the background is featureless in the range of 300 $\mathrm{cm}^{-1}$ to $1800 \mathrm{~cm}^{-1}$. 
(a)

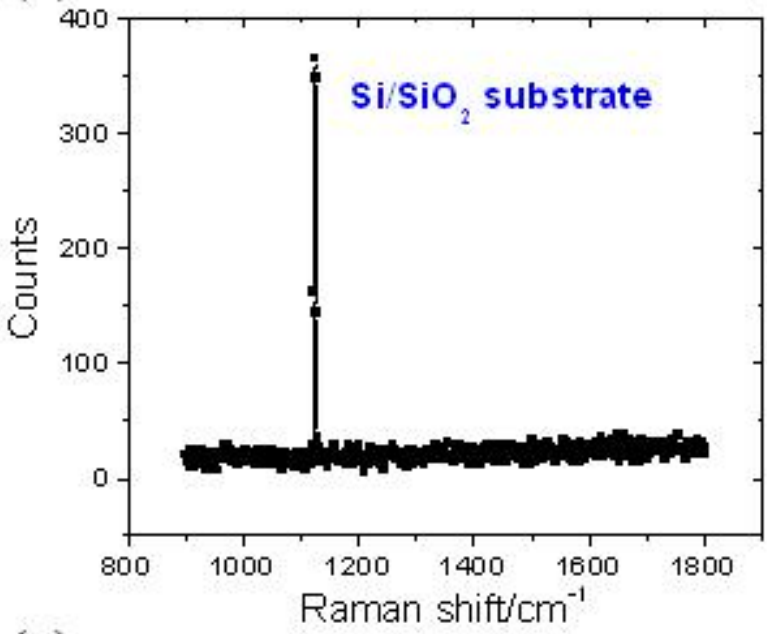

(c)

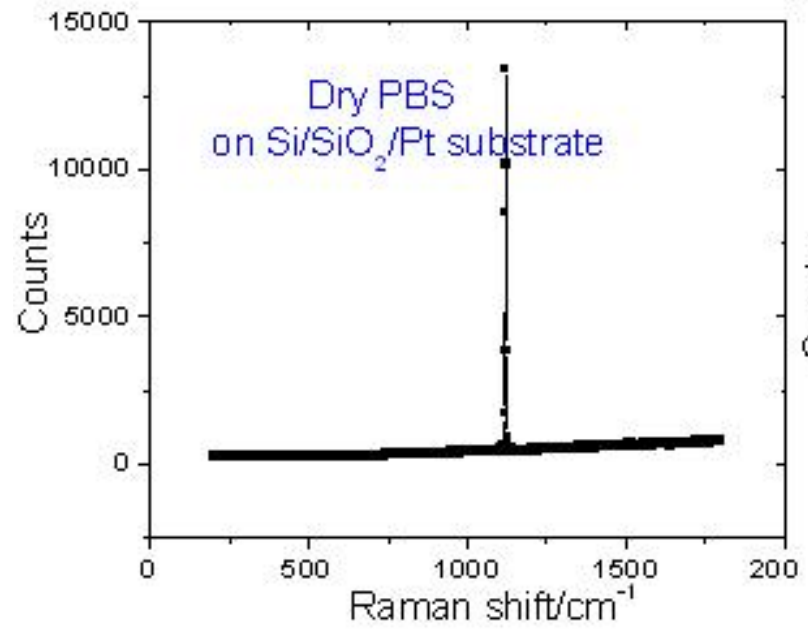

(b)

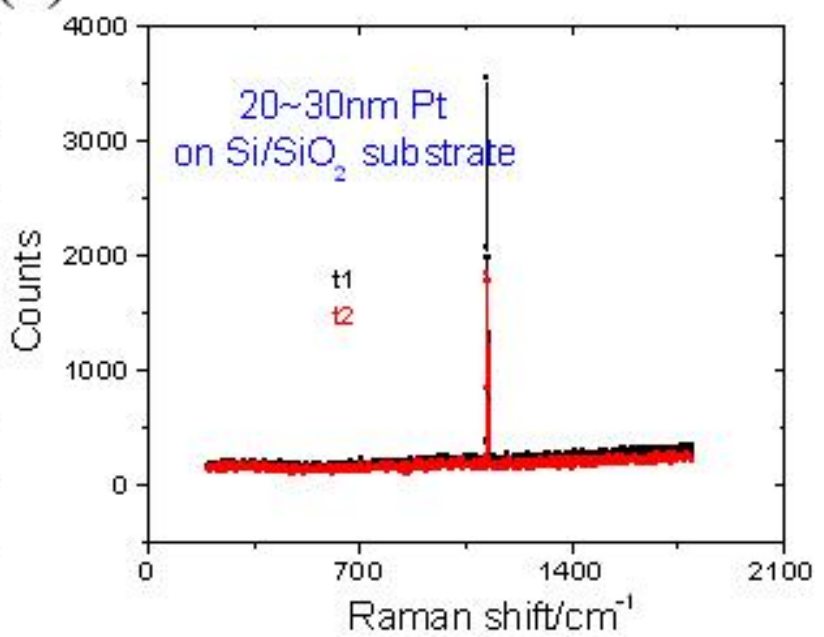

(d)

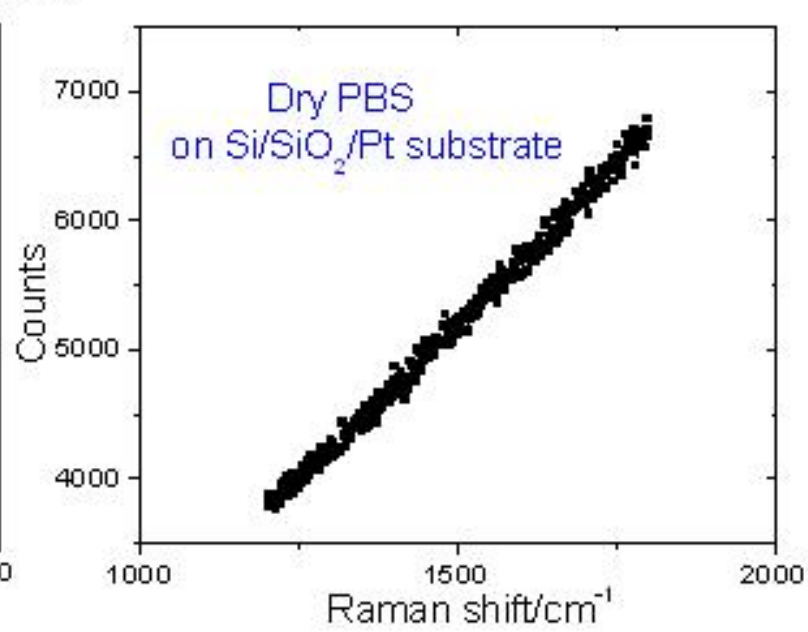

Figure 6.6 Background checking. (a) $\mathrm{Si} / \mathrm{SiO} 2$ substrate. (b) Pt thin film on $\mathrm{Si} / \mathrm{SiO} 2$ substrate. (c) Dry PBS on $\mathrm{Si} / \mathrm{SiO} 2 / \mathrm{Pt}$ substrate. (d) Dry $\mathrm{PBS}$ on $\mathrm{Si} / \mathrm{SiO} 2 / \mathrm{Pt}$ substrata at different conditions.

\subsubsection{Apomyoglobin}

As a reference, Raman spectroscopy was performed for apomyoglobin (myoglobin without the heme group) using a $514 \mathrm{~nm}$ excitation at $250 \mathrm{~mW}$ power. The spectrum was recorded with 30 seconds accumulation and repeated 5 times. The scan range was 1200-1800 $\mathrm{cm}^{-1}$. Two kinds of Raman spectra were compared (Fig. 6.7): (1) before cooling down (thin film); (2) after cooling down to $2.5 \mathrm{~K}$ for 6 hours and then warming up (thin film). The fact that the data were identical show that the protein is unaffected by thermal cycling. 


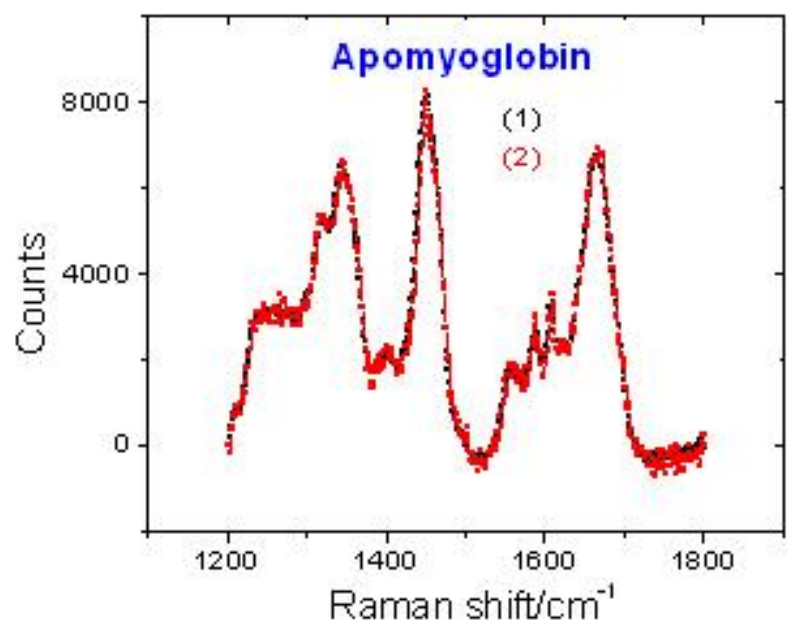

Figure 6.7 Raman spectroscopy for apomyoglobin. The black line shows the Raman spectra (1)(before cooling), the red line shows the Raman spectra (2) (after cooling).

\subsubsection{Myoglobin}

For the myoglobin samples the spectrum was recorded with 30 seconds accumulation and averaged 15 times. The laser power was adjusted to $5 \mathrm{~mW}$. The scan range was 1200-1800 $\mathrm{cm}^{-1}$. Four types of Raman spectra were taken (Fig. 6.8): (1) before cooling down (thin film); (2) after cooling down to $\sim 5.5 \mathrm{~K}$ for two days and then warming up (thin film); (3) after dropping some PBS on the sample after warming up; (4) with myoglobin in PBS at room temperature without cooling down.

(a)

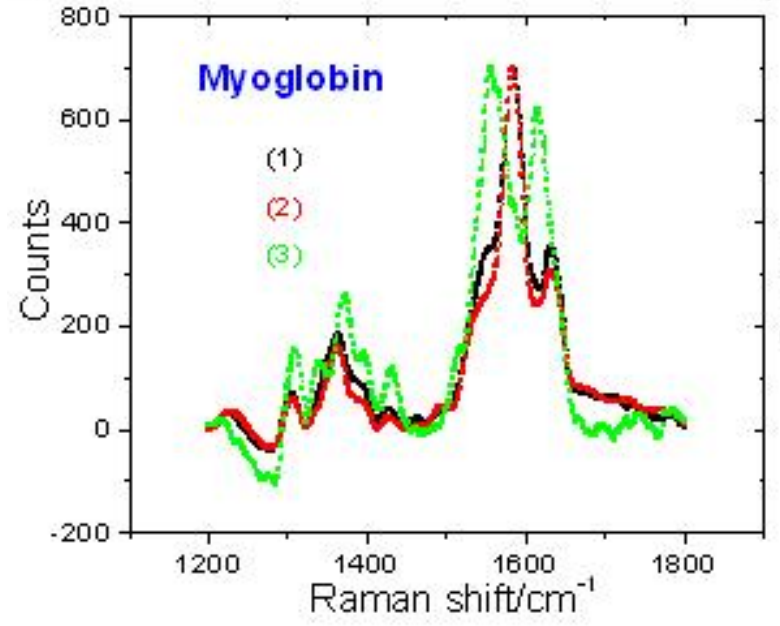

(b)

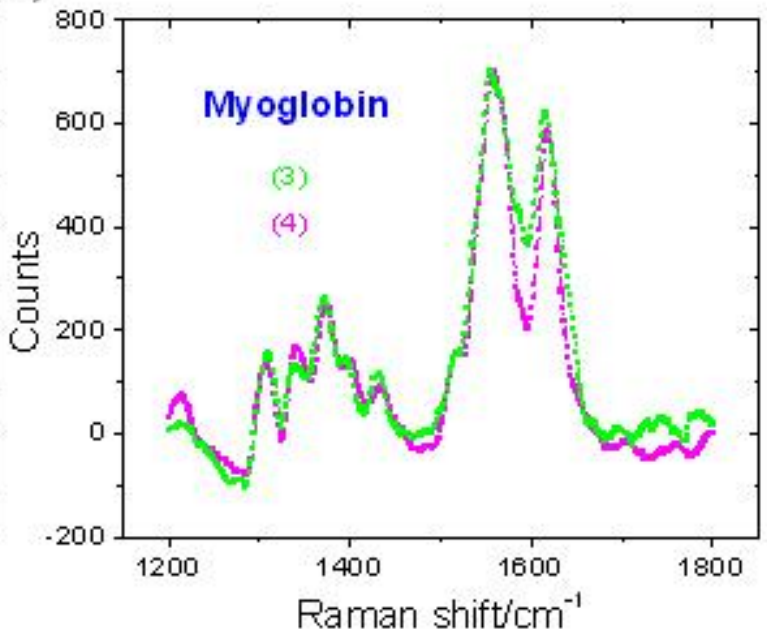

Figure 6.8 Raman spectroscopy for myoglobin. The black line shows the Raman spectra (1)(before cooling). The red line shows the Raman spectra (2) (after cooling). The green line shows the Raman spectra 
(3)(drop PBS again after cooling down). The pink line shows the Raman spectra (4)(Mb in PBS and never cool down).

The Mb Raman spectra can be explained using the heme structure in Mb. Figure 6.9 shows structural diagrams of the heme group in $\mathrm{Mb}$ for different situations. The case where $\mathrm{Mb}$ bound with water is shown in Fig. 6.9(a). After the Mb is dry, the heme iron loses the water and is bound to the amino acid His (Fig. 6.9(b)) so that the Fe spin state changes from high spin to low spin. ${ }^{75}$ This causes the main Raman peaks to shift because they are sensitive to the spin state of the heme iron. After cooling down and warming up, and dropping PBS into the dry $\mathrm{Mb}$, the Raman peaks moved to the initial positions. This means that the heme iron re-binds with water changing its low spin configuration to the high spin configuration. Because this is a clearly reversible process, these data show that the protein heme group does not denature during the cooling process.

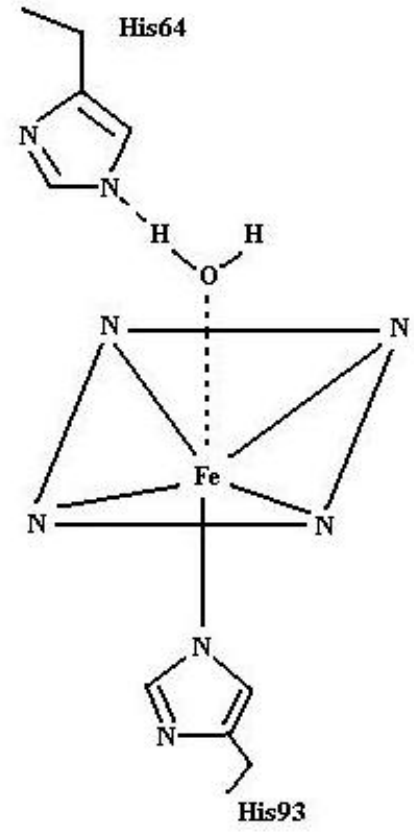

(a)

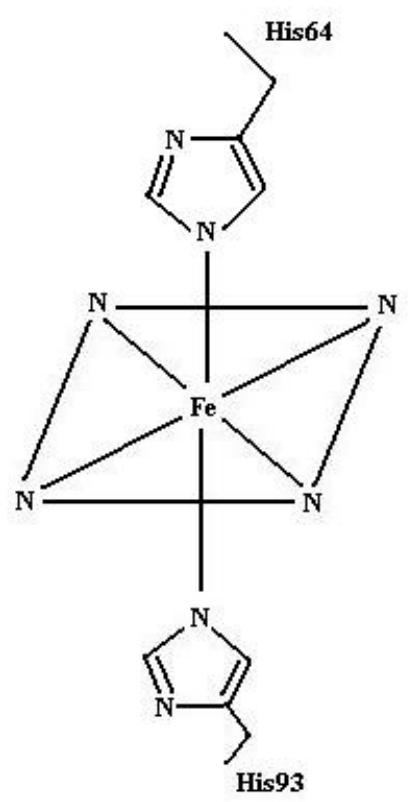

(b)

Figure 6.9 The heme structural diagrams of (a) Mb in PBS; (b) dry Mb film (revised from Ref. 75). 


\subsection{Summary}

In this chapter, protein vibrations treated as a simple diatomic oscillator and basic concepts of Raman spectroscopy were discussed. Raman scattering results ( $\alpha-\beta$ bands with the $514 \mathrm{~nm}$ excitation laser) show that myoglobin is still active after cooling down to $\sim 5 \mathrm{~K}$ for two days and then warming up to room temperature. Therefore, it is likely that myoglobin can retain its ability to undergo redox reactions at cryogenic temperatures. 


\section{Chapter 7}

\section{Electron Transfer by Apomyoglobin}

\subsection{Introduction}

Apomyoglobin is composed of 153 amino acids bound together to form a polypeptide chain. Experiments have found that the conductance of single peptide chains decrease with molecular length. ${ }^{41,76}$ There are 19 kinds of common amino acids in apomyoglobin, but there are no cysteine (Cys, C) groups. ${ }^{5}$ There are four groups of amino acids: nonpolar amino acids, polar uncharged amino acids, acidic amino acids with negatively charged $\mathrm{R}$ groups and basic amino acids with positively charged $\mathrm{R}$ group. The behavior and reactivity of specific $\mathrm{R}$ groups affect the electrical properties of the protein and determine its biological functionality. Recent research show that tryptophan, one kind of common amino acids, can accelerate electron flow through proteins. ${ }^{77,78}$

The aim of this work is to investigate the electron transport mediated by the heme group of myoglobin proteins. Apomyoglobin (myoglobin without the heme group) was used as a reference in our experiments.

In this chapter, the mechanism of electron transfer by apomyoglobin will be analyzed and one-dimensional conductance will be discussed. Results of conductance measurements of apomyoglobin at $77 \mathrm{~K}$ and $\sim 5 \mathrm{~K}$ will also be presented and analyzed.

\subsection{Electron Transfer Mechanism}

As introduced in the Chapter 2, the single electron transfer mechanism relies on elastic and inelastic tunneling mediated by vibrational excitations. However, because apomyoglobin does not contain a heme group, there is no elastic tunneling via heme group orbital energy levels. Inelastic tunneling measurements of vibrational spectra for some amino acids sandwiched in $\mathrm{Pb}$-based tunnel junctions have been performed at $4.2 \mathrm{~K}$ by measuring the second derivative of the current $\left(d^{2} I / d V^{2}\right){ }^{32}$ Since only a small fraction of electrons tunnel inelastically, the conductance from inelastic tunneling is too small to be conveniently detected. ${ }^{31}$ 
Elastic tunneling process can occur as a combination of tunneling through empty space and tunneling through hydrogen bonds. ${ }^{2} 79$ Here the repulsions from two or more covalent bonds or spaces in a row within the protein act as potential barriers for incident electrons. The attraction created by hydrogen bonds (the proton) is a potential well for electron tunneling. In this case, resonant tunneling would occur for certain energies of incident electrons, similarly to what happens in semiconductor superlattices. ${ }^{80}$ The simplest example is the well surrounded by two barriers ${ }^{34}$ discussed in the Chapter 2. Figure 7.1 show the possible mechanism for electron resonant tunneling through apomyoglobin. This will show up in the conductance vs. bias voltage characteristics as one or more conductance peaks.

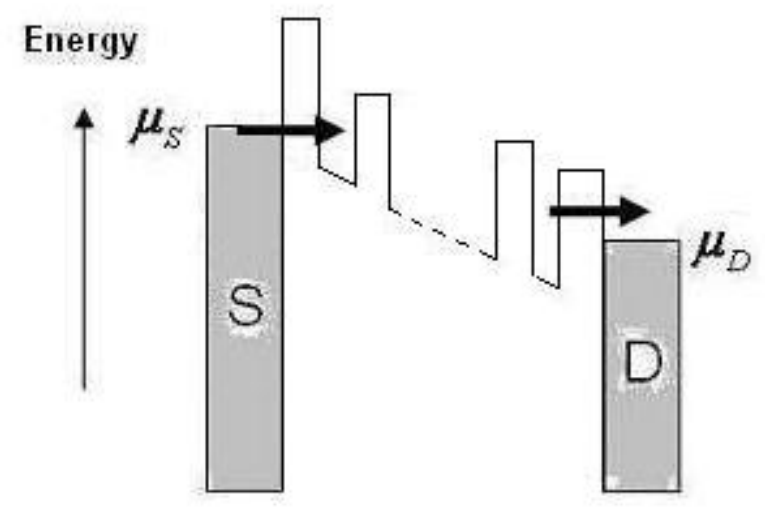

Figure 7.1 The possible mechanism for electron resonant tunneling through apomyoglobin.

\subsection{One-dimensional Conductance}

The conductance $G$ of a one-dimensional system due to elastic scattering of an obstacle can be expressed by Landauer's formula ${ }^{81,82,83,84}$

$$
G=\left(e^{2} / \pi \hbar\right) T / R,
$$

where $T$ and $R$ are the transmission and reflection coefficients, respectively.

A number of papers have argued that conductance $G \sim T$ or $G \sim T /(1-T)$ after the equation (7.1) is found. ${ }^{82,85,86}$ One explanation is that when deriving Equation (7.1), the system is connected to a classical wire, behaving as a current source. ${ }^{85}$ For a onedimensional chain without interacting (spinless) electrons, the DC conductance is found to be ${ }^{85}$ 


$$
G=\left(e^{2} / \pi \hbar\right) T,
$$

where $T$ is the transmission coefficient.

By contrast, equation (7.2) yield quantum conductance for resonance tunneling $T$ $=1$ and equation (7.1) yield an infinite conductance for $T=1$. Equation (7.2) has turned

out to have a very significant range of applicability. ${ }^{82,87}$ Later, Landauer's formula was expanded to higher dimensions such that ${ }^{82}$

$$
G=\left(e^{2} / \pi \hbar\right) \operatorname{Tr}\left(t t^{+}\right),
$$

where $t$ is the transmission matrix of the device. The trace in the equation (7.3) represents a sum over the conduction channels of an ideal conductor.

\subsection{Some Results at $77 \mathrm{~K}$}

In the apomyoglobin experiments, $125 \mathrm{Pt}$ protein-coated junctions were broken by electromigration at $77 \mathrm{~K}$. Of these, 20 junctions had conductance peaks and nine of these had a very weak response to the electric field applied through the gate electrode. Figure 7.2 shows typical results from one apomyoglobin sample with a single conductance channel. Figures 7.2(a) and 7.2(b) show $I-V$ characteristics during the junction-breaking process. Figure 7.2(b) shows that this junction was broken four times until steps in the $I$ $V$ graph were found. Figure $7.2(\mathrm{c})$ is the conductance measured at $77 \mathrm{~K}$ with zero gate voltage which shows one conductance peak at $V_{\text {bias }}=-15 \mathrm{mV}$. This conductance peak may display the resonance tunneling behavior described by Equation 7.2. The data became very noisy at higher bias voltages, indicating that perhaps the protein's interaction with the applied electric field ( $\left.V_{\text {bias }}\right)$ made its conformation unstable. At $77 \mathrm{~K}$, however, the conductance features remained more or less constant as a function of $V_{\mathrm{G}}$ (Fig. 7.2(d)). Figure 7.2(e) shows how this conductance peak changed with temperature. The temperature dependence is evidence that this conduction mechanism is due to resonant tunneling because of the amplitude of the conductance peak decreases as the temperature increases as $\mathrm{G} \sim 1 / T^{88,}{ }^{89}$ This is illustrated in Fig. 7.2(f), where the amplitude of the peak, fitted to a Gaussian line shape, is plotted as a function of $1 / T$ which yields a straight line fit. Figure 7.2(g) shows that the conductance peak changes slightly with gate voltage after cooling down again. Nevertheless, as Fig. 7.2(h) 
illustrates, the background conductance changed after warming up to $150 \mathrm{~K}$ and cooling back down to $77 \mathrm{~K}$. This suggests that the protein conformation or orientation may have changed after cycling the temperature. In contrast, Fig. 7.2(h) also shows two conductance channels (peaks) after applying $V_{G}=+9.0 \mathrm{~V}$ (t3). In this case, the conductance profile changed drastically, suggesting that a substantial change in the conformation of the protein occurred. These experiments illustrate the importance of the effects of the conformation of the protein adopted when bound to the electrical contacts on the measurements. 

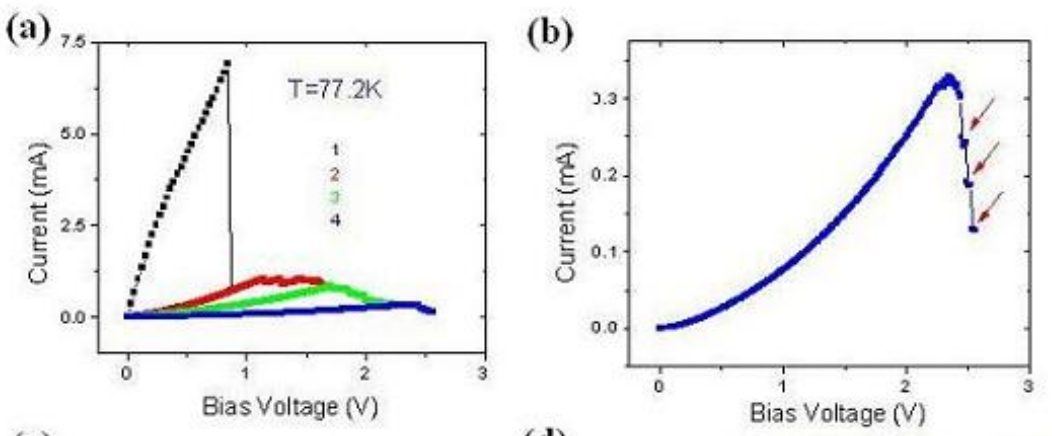

(c)
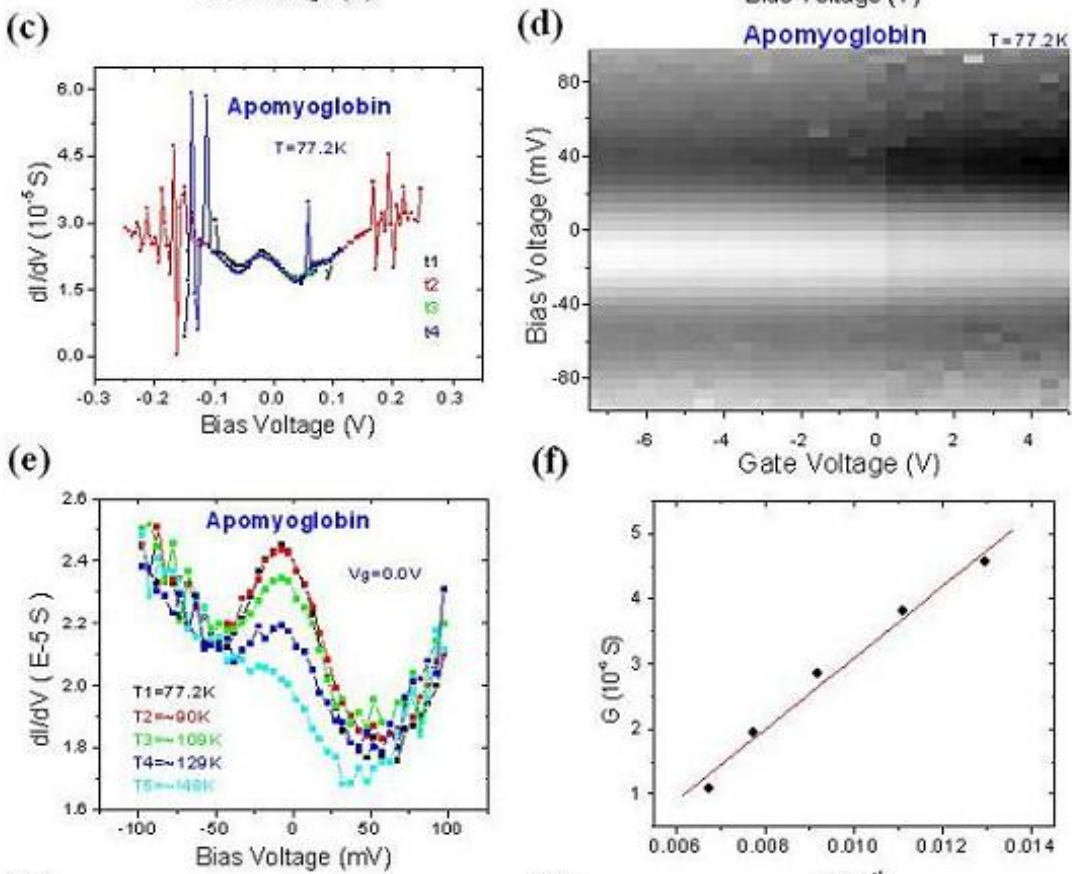

(f)
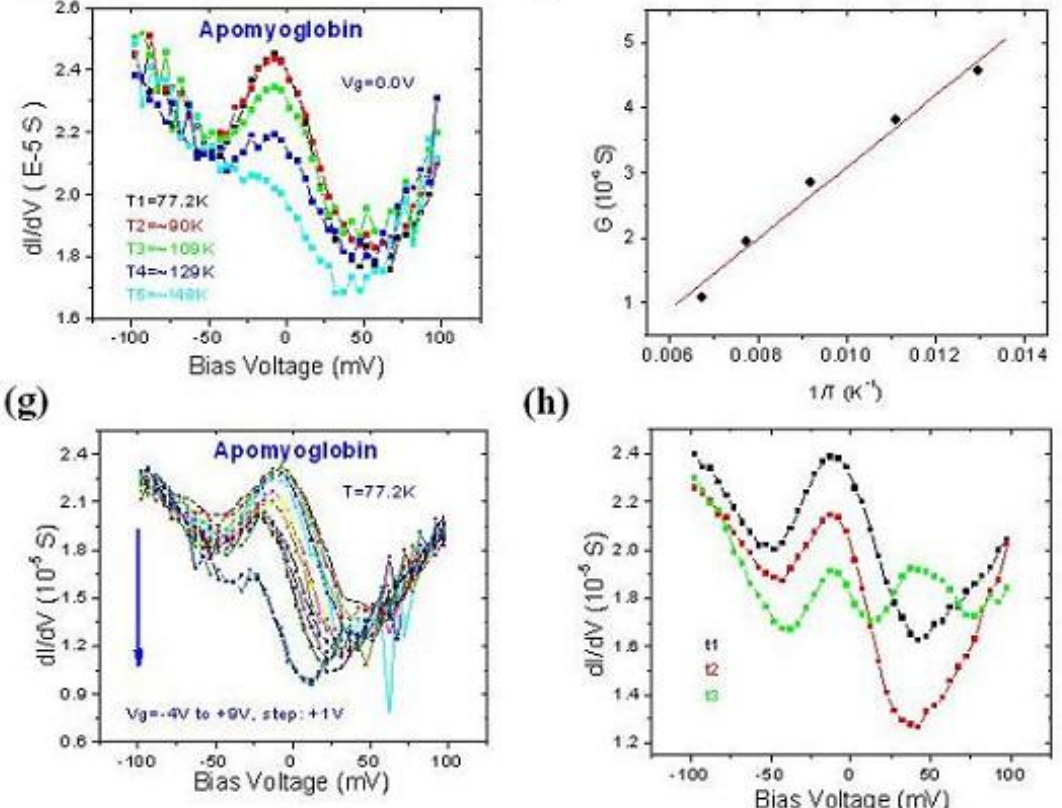

(h)

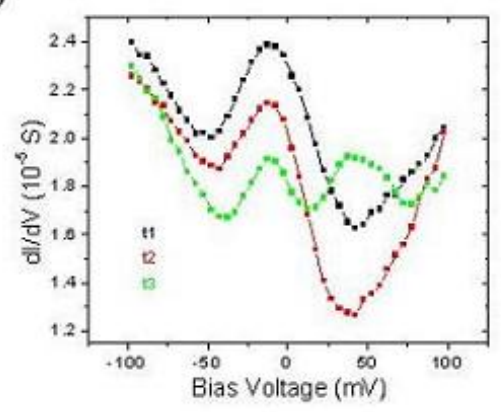

Figure 7.2 One apomyoglobin sample at $77 \mathrm{~K}$. (a) shows $I-V$ characteristics during the junction-breaking process. (b) shows that this junction was broken four times until steps in the $I-V$ graph were found. (c) is the conductance measured at $77 \mathrm{~K}$ with zero gate voltage which shows one conductance peak at $V_{\text {bias }}=-15$ $\mathrm{mV}$. (d) shows the conductance features remained more or less constant as a function of $V_{\mathrm{G}}$. (e) shows how this conductance peak changed with temperature. (f) shows the amplitude of the peak is plotted as a function of $1 / T$ which yields a straight line fit. (g) shows that the conductance peak changes slightly with gate voltage after cooling down again. (h) illustrates that the background conductance changed after warming up to $150 \mathrm{~K}$ and cooling back down to $77 \mathrm{~K}$. The black curve (t1) shows the initial conductance, the red curve (t2) shows the conductance after cooling down and warning up, and the blue one (t3) shows the final conductance after applying $V_{G}=+9.0 \mathrm{~V}$. 
(a)

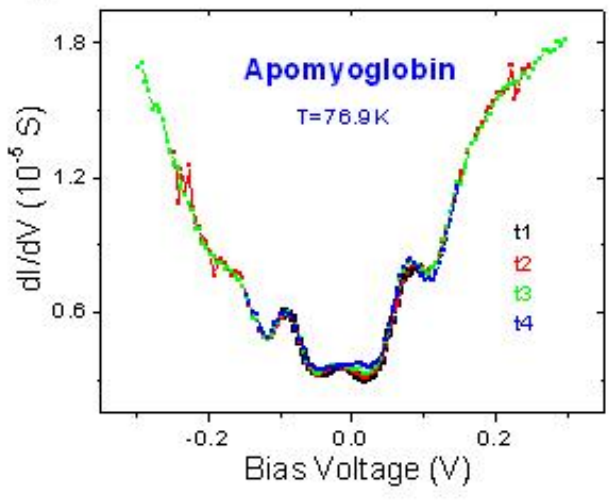

(b)

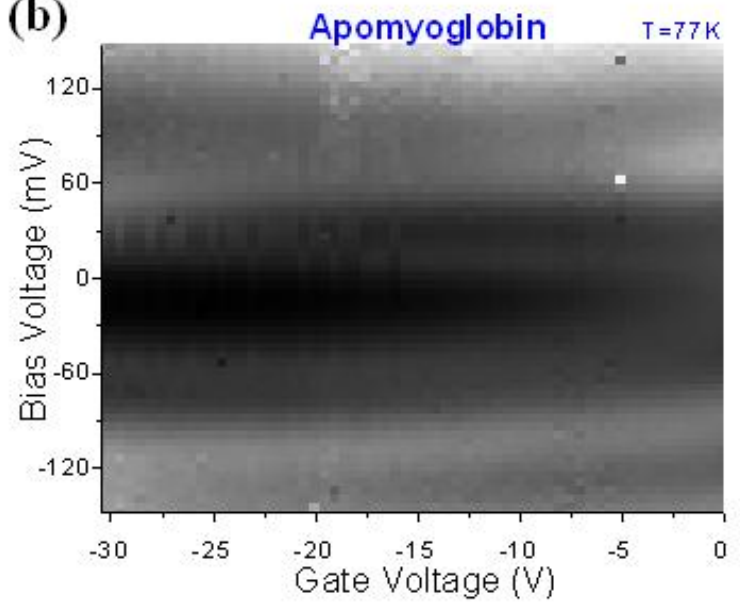

Figure 7.3 One apomyoglobin sample at $77 \mathrm{~K}$. (a) shows that some conductance peaks can be found after breaking the junction. (b) shows the dependence of there conductance lines on bias and gate voltages.

Figure 7.3(a) shows that some conductance peaks can be found after breaking the junction of another sample at $77 \mathrm{~K}$. Figure 7.3(b) shows the dependence of there conductance lines on bias and gate voltages. These conductance lines could be due to the incident electron resonance tunneling by potential barriers. Once again, there is little dependence of the conductance on the gate voltage.

\subsection{Some Results at $\sim 5 \mathrm{~K}$}

Forty-three apomyoglobin samples were measured at 5.5 K. Fifteen of them had conductance peaks and two had a very weak field (gate voltage) dependence. For one of the latter two samples, Fig. 7.4(a) shows the existence of some conductance peaks after breaking the junction. Figure 7.4(b) shows that their conductance lines changed slightly with gate voltage. The red arrow indicates where one of the conductance peaks splits with gate voltage. Figure 7.4(c) shows the temperature dependence of these conductance lines. The strong degradation of the features with increasing temperature indicates that tunneling is the mechanism that dominates the conduction.

As mentioned above, the conformation of the protein is not likely to be the same in different devices. Therefore, the asymmetrical sequence of amino acids in the narrowest part of electrodes could significantly affect the conductance measurements. 
(a)

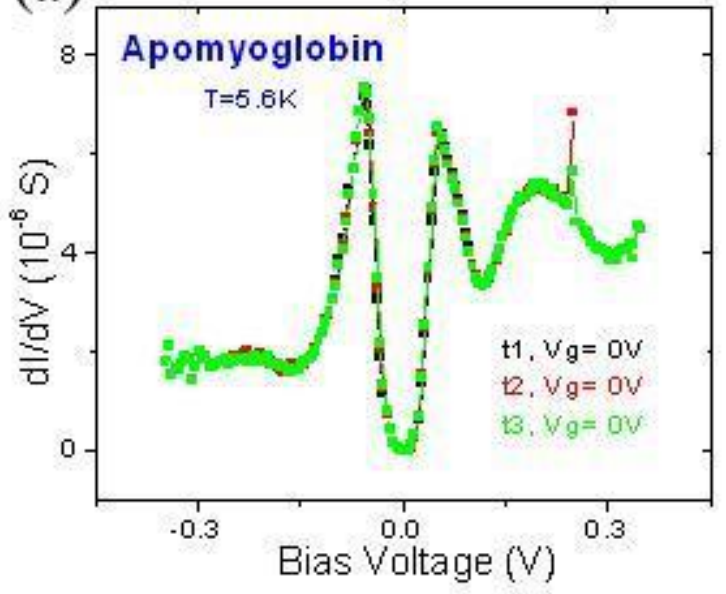

(c)

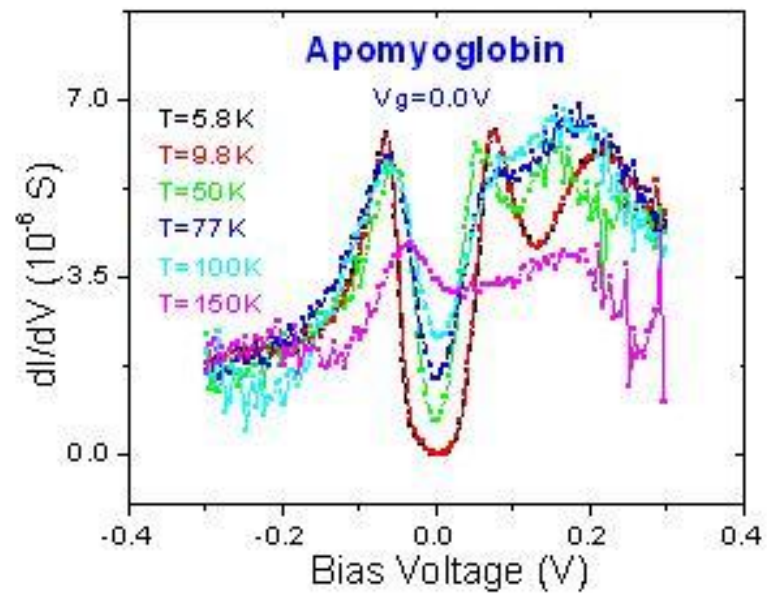

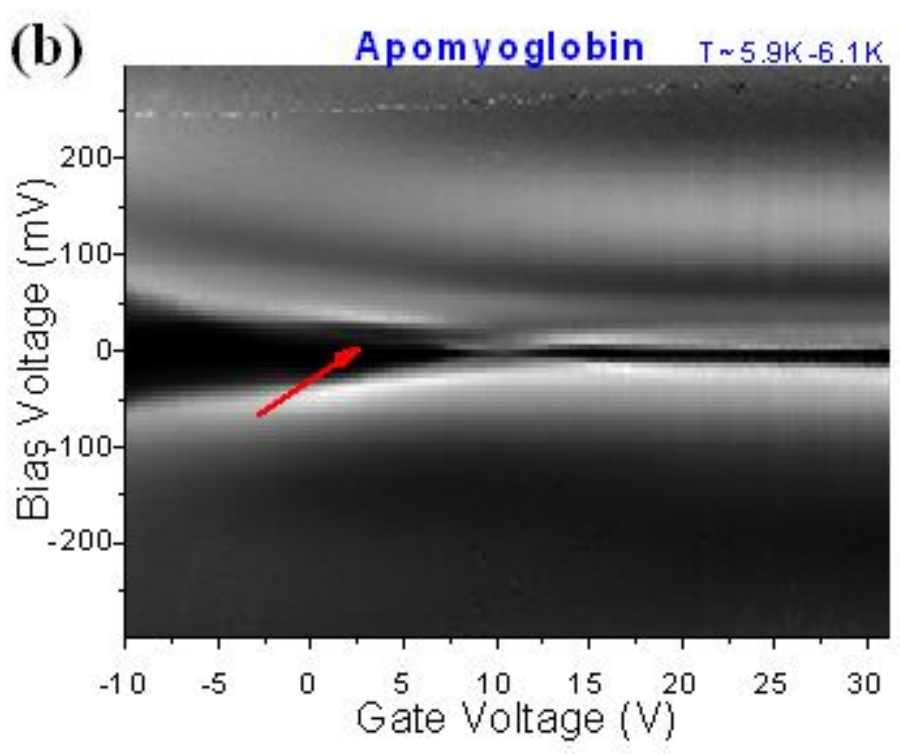

Figure 7.4 One apomyoglobin sample at $6 \mathrm{~K}$. (a) Reproducible conductance peaks after breaking the junction. (b) Conductance lines changed slightly with gate voltage. The red arrow indicates where one of the conductance peaks splits with gate voltage. (c) Temperature dependence of these conductance lines.

\subsection{Summary}

The possible mechanisms of electron transport by apomyoglobin were discussed. Our experimental results show that resonance tunneling was observed in some samples. The orientation of the protein is not likely to be the same in different devices and therefore different devices have different tunneling spectra, but in all devices the dependence on gate voltage was weak. 


\section{Chapter 8}

\section{Electron Transfer by Myoglobin}

\subsection{Introduction}

Myoglobin is a protein found in muscles containing a heme group. It has been used in electrochemical studies as a model system because its crystal structure has been determined by high-resolution X-ray crystallography. ${ }^{2,90}$ Although its function is oxygen storage and transport, it can be used to study enzyme electron transfer. ${ }^{4,91}$ The heme group has a porphyrin ring with a $\mathrm{Fe}$ ion redox center that has two oxidation states: the ferrous $\left(\mathrm{Fe}^{2+}\right)$ and the ferric $\left(\mathrm{Fe}^{3+}\right)$ states. The $\mathrm{Fe}$ ion changes valence state depending on the whether an electron is accepted or given up by the protein.

In this chapter, some results of cyclic voltammetry (CV) measurements in $\mathrm{Mb}$ films previously performed by others will be reviewed. Then the mechanism of electron transport by myoglobin, and the experimental results will be discussed.

\subsection{Cyclic Voltammetry (CV) for Mb Film}

Myoglobin was chosen not only for its relatively small size $(4.4 \mathrm{~nm} \times 4.4 \mathrm{~nm} \times$ $2.5 \mathrm{~nm}$ ) with respect to other proteins, ${ }^{5}$ which is desirable to increase the electron tunneling probability, but also for its well-studied electrochemical properties. CV measurements in $\mathrm{Mb}$ films (from horse skeletal muscle) indicate that the redox potential is at $-0.34 \mathrm{~V}$ measured with a scan rate $0.1 \mathrm{~V} / \mathrm{s}$ in a $\mathrm{pH}=7.4$ buffer solution (Fig. 8.1) ${ }^{75}$ Raman spectra indicate that this kind of $\mathrm{Mb}$ films is in the ferric state. ${ }^{75}$ Therefore, when the protein is placed between two metallic electrical contacts (Fig. 8.2(a)), its electrochemical potential $\mu\left(\mathrm{Fe}^{3+} \rightarrow \mathrm{Fe}^{2+}\right)$, or vacant redox level, is above the Fermi level of the electrodes when no bias and gate voltages are applied (Fig. 8.2(b), $\mu=E_{0}$ for $V_{\text {bias }}=$ $\left.V_{\text {gate }}=0\right)$. 


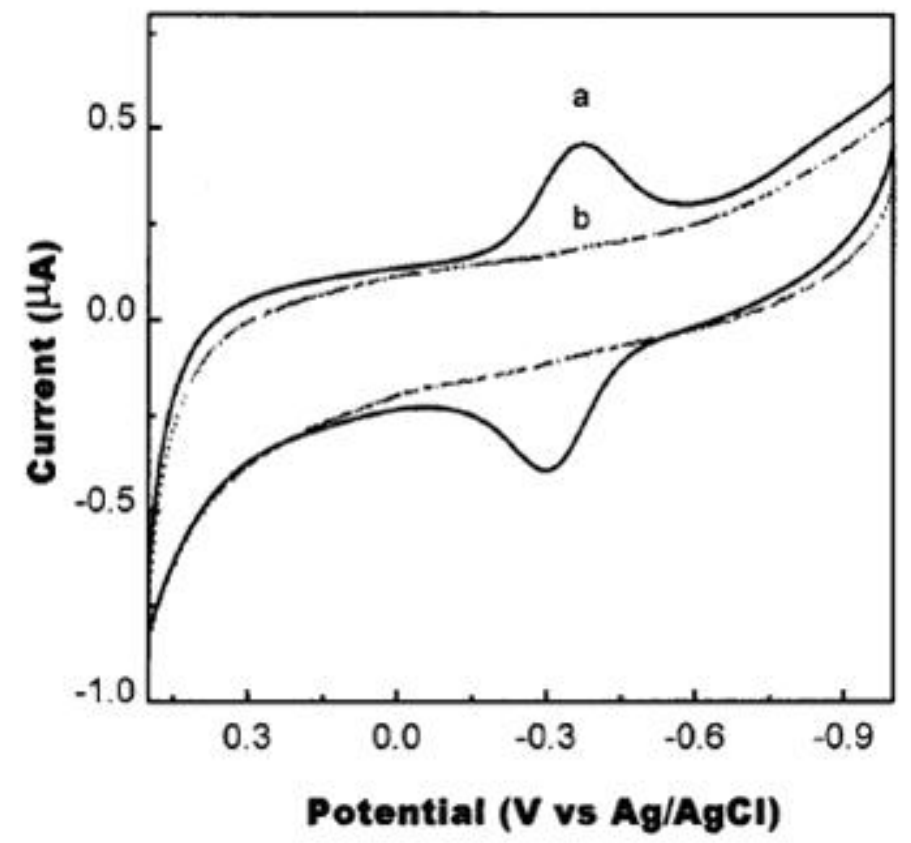

Figure $8.1 \mathrm{CV}$ of (a) the $\mathrm{Mb}$ film electrode in a $\mathrm{pH} 7.4$ phosphate buffer and (b) a bare glassy carbon electrode in a buffer solution containing $5 \times 10^{-4} \mathrm{M}$ metMb. Scan rate $=0.1 \mathrm{~V} / \mathrm{s}$. (from Ref. 75).

(a)

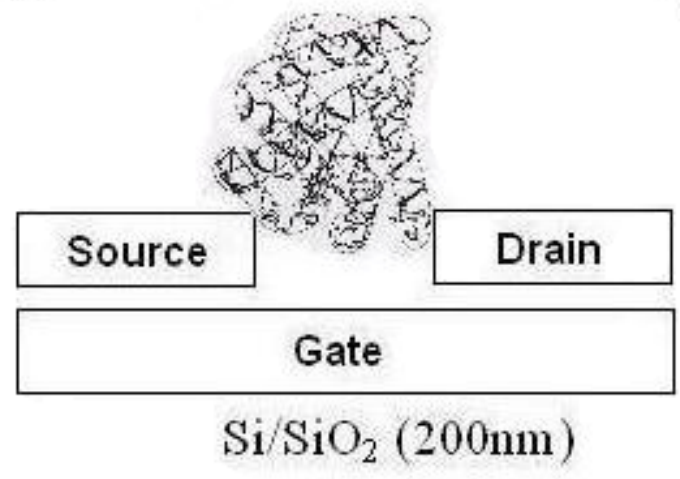

(b)

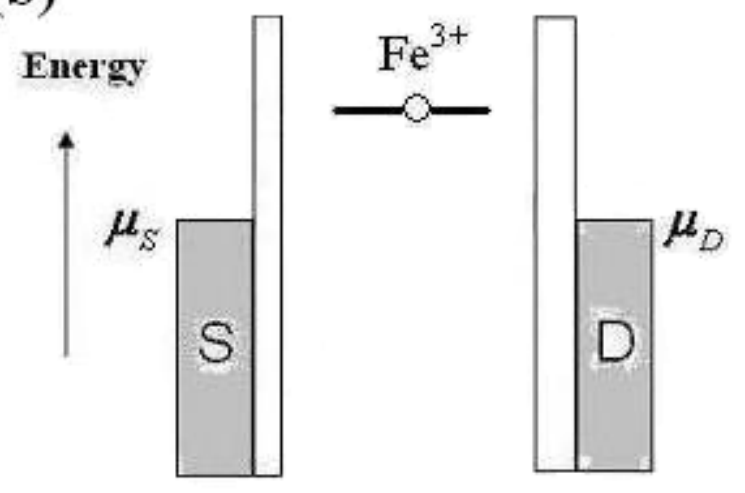

Figure 8.2 (a) Protein measurement device. (b) There is an electrochemical potential $\mu\left(\mathrm{Fe}^{3+} \rightarrow \mathrm{Fe}^{2+}\right)$ above the Fermi level. Just this level is drawn for simplicity (S: source, D: drain).

An important question is what is the redox potential $E_{0}$ for a single protein. The midpoint potential at $-0.34 \mathrm{~V}$ was obtained from $\mathrm{Mb}$ film measurements that averaged over a large number of proteins. In addition, $\mathrm{CV}$ measurements show that the midpoint potential becomes less negative as the solution $\mathrm{pH}$ is decreased (Fig. 8.3). ${ }^{92}$ Indeed, the midpoint potential of myoglobin is $-0.15 \mathrm{~V}$ in a $\mathrm{pH}=7.1$ buffer. $^{93}$ The material used for the electrode has a strong influence on the redox potential. ${ }^{4}$ These dependencies may be 
different for single proteins in nanometer electrodes because of the particular protein conformation $^{94}$ and the local electrostatic environment (i.e. the interface between protein and metal electrode).

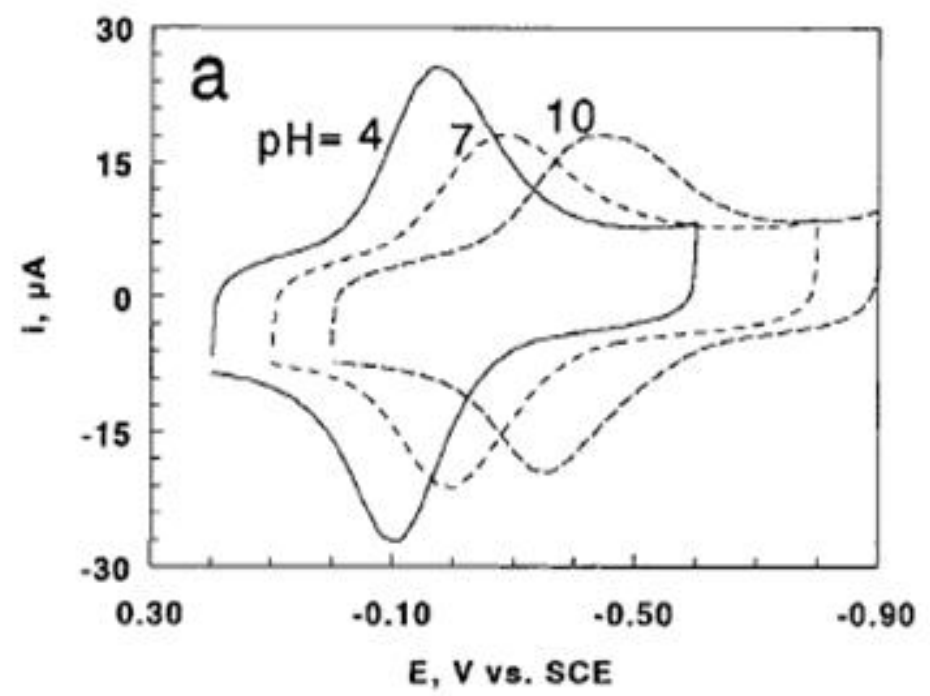

Figure 8.3 CV at $2 \mathrm{~V} / \mathrm{s}$ in buffers containing $100 \mathrm{mM} \mathrm{NaBr}$ of Mb-DDAB on PG electrode (from Ref. 92).

\subsection{The Charge State and Charging Energy}

The charge state and charging energy of the heme iron have been introduced above by some electrochemical measurements of MB film. It is difficult to figure out its charge state and charging energy in the transistor geometry just by electrochemical data. Previous work has shown that there is a charging energy reduction in the transistor environment due to image charges in the metal and the polarizability of the molecule ${ }^{13}$, as well as shifts in the charge degeneracy point on an absolute scale from device to device. These factors all make charge state assignment more complicated than direct comparison to electrochemical data. From this point of view, the free heme iron has a large gap between the HOMO and LUMO levels as shown in Fig. 8.4(a). When the protein is trapped in the noble metal electrodes, the energy spacing between neighboring electrochemical potentials becomes smaller (shown in Fig. 8.4(b)) due to the moleculeelectrode interaction. ${ }^{13}$ 
(a)
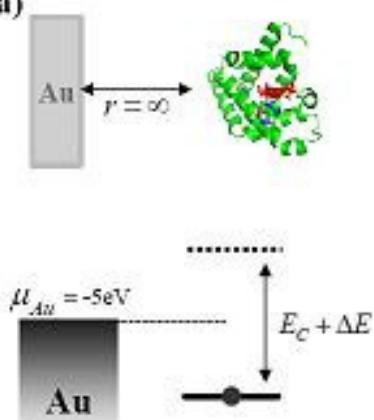

(b)
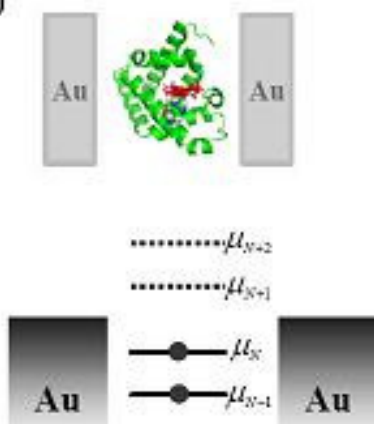

Figure 8.4 The electrochemical potential of protein. (a) for the isolated protein; (b) for the trapped protein.

\subsection{Electron Transfer Mechanism}

There are several factors that influence the redox potential of a molecule. ${ }^{94,95}$ One way is by applying a gate voltage through a back gate electrode, which is one of the three electrodes in a SET three-terminal device (Fig. 8.2(a)). ${ }^{13,96}$ In normal transistor geometries, the metal ion charge state in the molecule can be tuned to the Fermi level of the electrodes by changing the back gate voltage, as Fig. 8.5(a) illustrates.

As discussed in the Chapter 1, electron transfer by a single protein has been probed using EC-STM. Two mechanisms for electron transfer (ET) by single redox metalloproteins in aqueous solutions at room temperature were mentioned. The resonant tunneling model implies that the electron transfer between the tip and the substrate is much faster than the relaxation time of the redox center. At cryogenic temperatures, the ET mechanism by redox metalloproteins is single electron transfer, with the accompanying Coulomb blockade effect, if the charging energy is much larger than the thermal energy. ${ }^{97}$ In this case, the conductance mechanism can also be regarded as sequential resonance tunneling. It is expected that the current is blocked at the Coulomb blockade region and conductance peaks will be found at the tunneling thresholds for transitions between two charges states, as has been observed in some small molecules with redox center. ${ }^{13,36}$ Nevertheless, for the two-step ET process, nuclear relaxation upon tunneling is still available due to conformational changes in the protein induced by the redox reaction. In particular, it is known that in heme-containing proteins redox

reactions cause significant conformational changes of the heme group. ${ }^{98}$ Therefore, the two-step ET transfer process is expected to be valid at low temperatures in these proteins. 
The electron transport theory of a quantum dot with a two-step tunneling process can be used to describe the electron transport of the device.

(a)

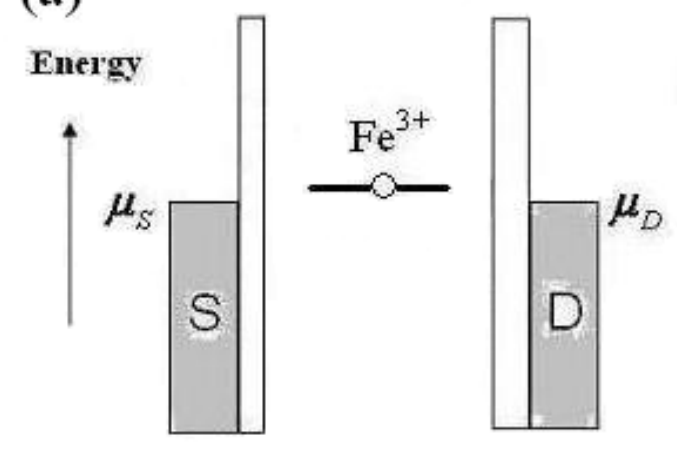

Redox level shift with Vgate

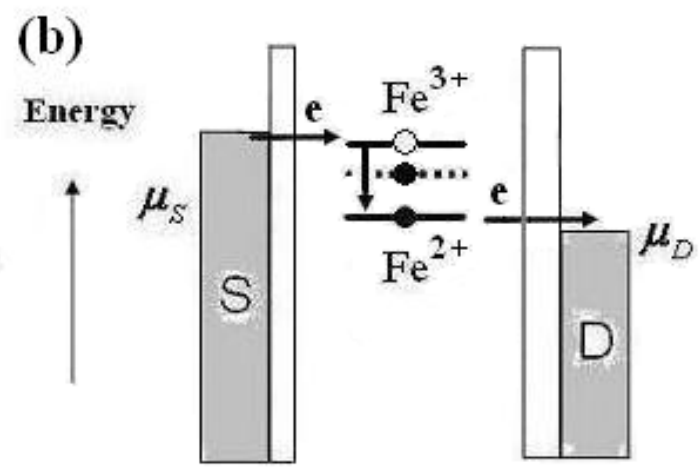

Two-step ET

Figure 8.5 Electron transport mechanism. (a) The redox level can be shifted to the Fermi level of the electrodes by the gate voltage. (b) Two-step electron transfer by protein.

For single electron transport at cryogenic temperature, the total tunneling time $\tau$ can be estimated from $\tau=|e| / I$, where $\tau \sim$ ps for a current $I \sim 10^{-7} \mathrm{~A}$. The protein relaxation time from the intermediate states to the ground state could range from picoseconds to seconds. ${ }^{99}$ Because of the redox properties of the Fe ion, as electrons tunnel into the protein, the $\mathrm{Fe}$ ion fluctuates between its two charged states (an empty level and an occupied level). If the relaxation time is long, the electron can resonantly tunnel through the protein with the protein remaining in the same intermediate state. In this case, Coulomb blockade behavior will show up in a differential conductance graph with its degeneracy point at zero bias and the crossing potential $\left(V_{C}\right)$ by tuning the gate voltage $\left(V_{g}\right)$ up to $V_{C}$. If the relaxation time is very short $(\sim \mathrm{ps})$, the protein can quickly relax from the intermediate state to its ground state, whose level may be below the Fermi level of electrodes, as Fig. 8.5(b) illustrates. In this case, the electron will be trapped in the protein, the current is blocked and Coulomb blockade behavior will not have a degeneracy point in the differential conductance graph. The calculations in Fig. 8.6 were performed using the rate equation ${ }^{13,36}$ for electron tunneling via discrete quantum states. 

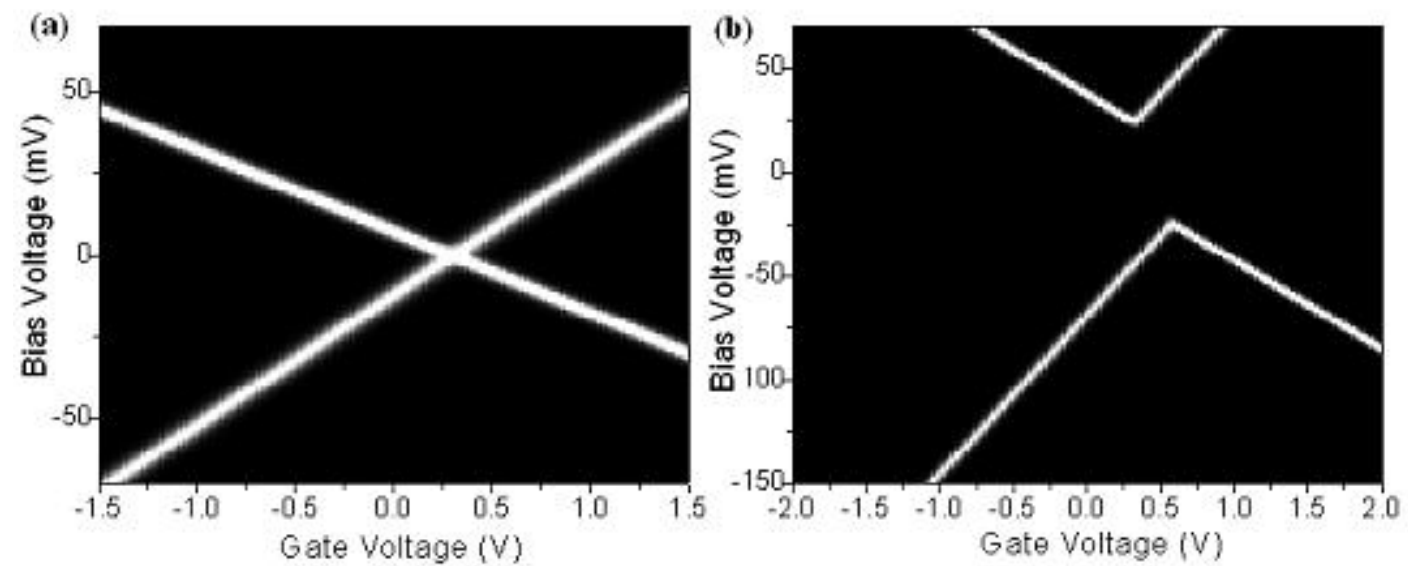

Figure 8.6 (a) Calculated differential conductance $d I / d V$ as a function of gate and bias voltages without the two-step process for $T=6 \mathrm{~K}, E_{0}=4.72 \mathrm{mV}, C_{D}: C_{S}: C_{G}=24: 40: 1$, tunneling rates $\Gamma_{S}=\Gamma_{G}=10 \mathrm{GHz}$. (b) Calculated differential conductance $d I / d V$ as a function of gate and bias voltages with a two-step process for $T=6 \mathrm{~K}, \delta E=25 \mathrm{mV}, E_{0}=25 \mathrm{mV}, C_{D}: C_{S}: C_{G}=12: 23.5: 1$, tunneling rates $\Gamma_{S}=\Gamma_{G}=10 \mathrm{GHz}$.

Although a single myoglobin protein could be viewed as a quantum dot, as discussed above, the question remains as to the importance of conduction through the amino acids around the heme group which could provide parallel conduction channels. The conductance channels could be resonant tunneling via the redox level or other electronic states in the protein, both of which could be enhanced by the vibrational states of the heme group and/or surrounding amino acid groups. It is well-known that the heme group is not at the center of the protein. It should be also noted that the orientation of the proteins in the gap can not be controlled through the processes of protein deposition and electromigration. Therefore, the asymmetrical structure and numerous orientations of the protein could significantly affect the conductance measurements (i.e., the relative importance of the different conductance channels) from sample to sample.

\subsection{Some Results at $77 \mathrm{~K}$}

A total of $96 \mathrm{Pt}$ junctions coated with myoglobin were broken by electromigration with feedback at $77 \mathrm{~K}$. Out of these, 23 had clear conductance peaks as a function of bias voltage. Three of these samples showed a clear dependence on the gate voltage. Figure 8.7 shows some results from one of these samples. Figure 8.7(a) shows the $I-V$ characteristics after breaking the junction several times until conductance peaks or steps were found as shown in Fig. 8.7(b). The red arrow in Fig. 8.6(a) indicates that the current increased even after the junction broke. In single molecular measurements, this is a 
signature of at least one molecule being incorporated into the gap. ${ }^{13,24}$ Figure 8.7(c) shows the dependence of the conductance of myoglobin on the gate voltage at $85 \mathrm{~K}-90$ $\mathrm{K}$. The conductance lines had a weak dependence on the gate voltage. One peak, indicated by the red arrow, split into two peaks with increasing gate voltage. This suggests that the split peak came from the overlay of two resonant tunneling processes. However, the heme group conductance is not evident because of the weak gate voltage dependence. This could be a result of the way the protein orients in the electrodes, which could favor transport through the amino acid shell rather than by resonant tunneling through the heme group, or simply due to the temperature of the sample, which tends to mask SET behavior. 
(a)

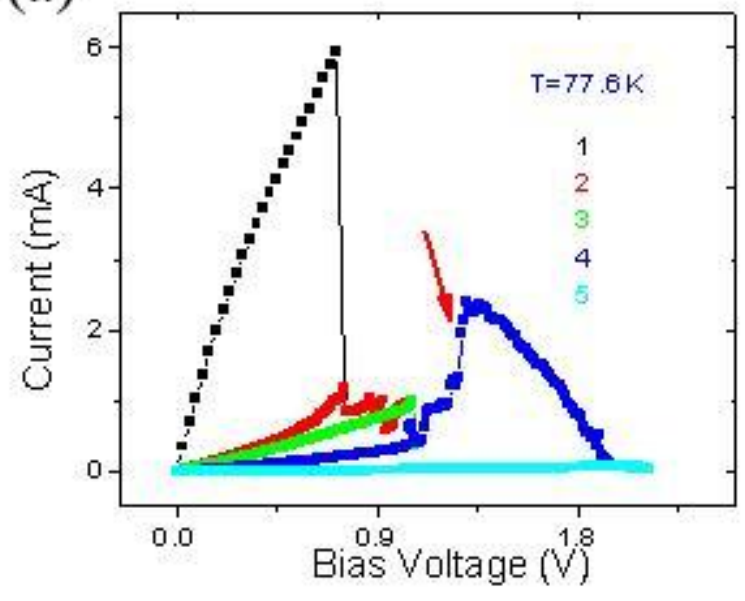

(b)

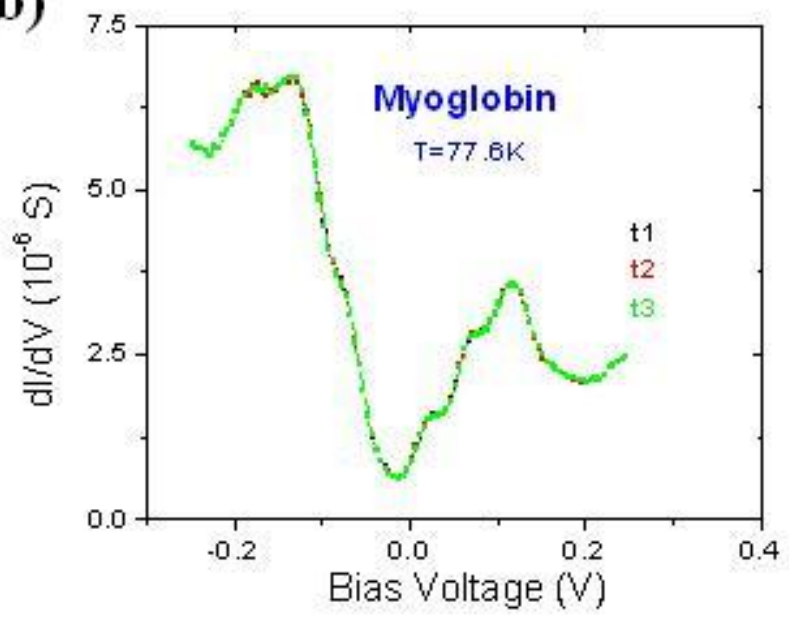

(c)

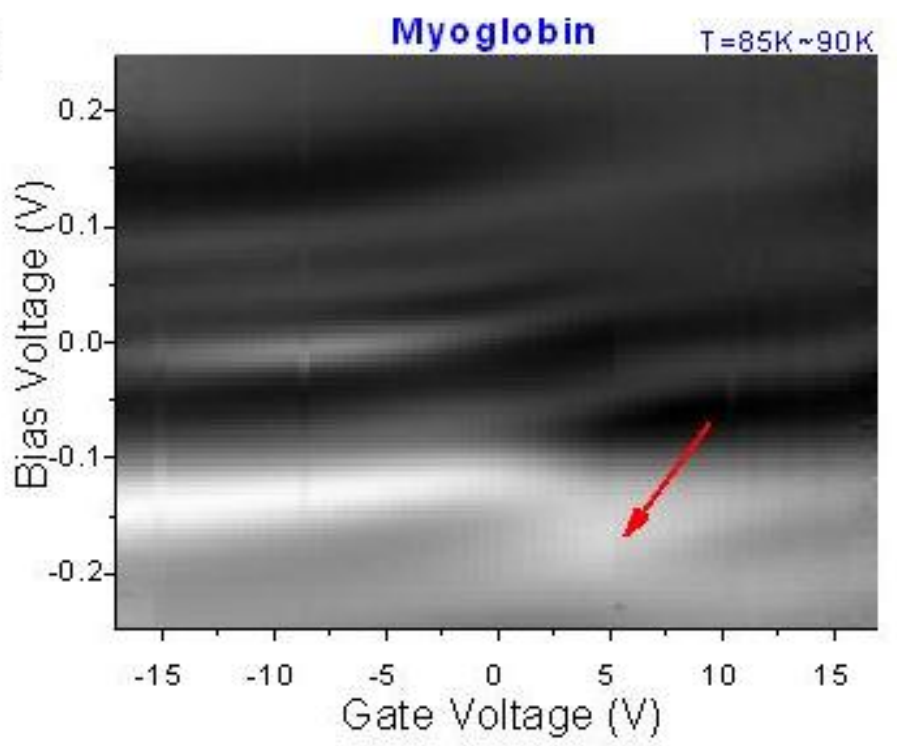

Figure 8.7 One myoglobin sample at $85 \mathrm{~K}$ (a) Breaking junction process; (b) Some peaks or steps can be found in $d I / d V_{\text {bias }}$ as function of bias voltage, $V_{\text {gate }}=0 \mathrm{~V}$; (c)Differential conductance $d I / d V_{\text {bias }}$ as function of gate and bias voltage at $85 \sim 90 \mathrm{~K}$ (gray scale from 7.0E-7 S [black] to $1.0 \mathrm{E}-5 \mathrm{~S}$ [white]).

Figure 8.8 shows some results from one $\mathrm{Mb}$ sample at $77 \mathrm{~K}$ made with an $\mathrm{AC}$ measurement technique. The conductance spectra of this sample were measured using a lock-in technique with a modulation signal. Figure 8.8(a) shows some conductance peaks found in the range of $V_{\text {bias }}= \pm 0.5 \mathrm{~V}$. The peaks were altered by the application of a gate voltage $(-20 \mathrm{~V},-30 \mathrm{~V},-40 \mathrm{~V})$. Figure 8.8(b) shows the dependence of the conductance of myoglobin on the gate voltage from $-22 \mathrm{~V}$ to $-34 \mathrm{~V}$ at $77 \mathrm{~K}$. It was not possible to map the whole conductance behavior because this sample was damaged at $V_{\text {gate }}=-35 \mathrm{~V}$. 
(a)

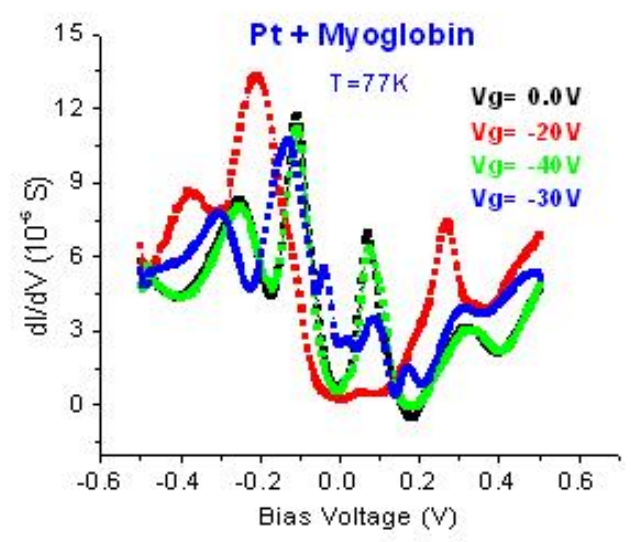

(b)

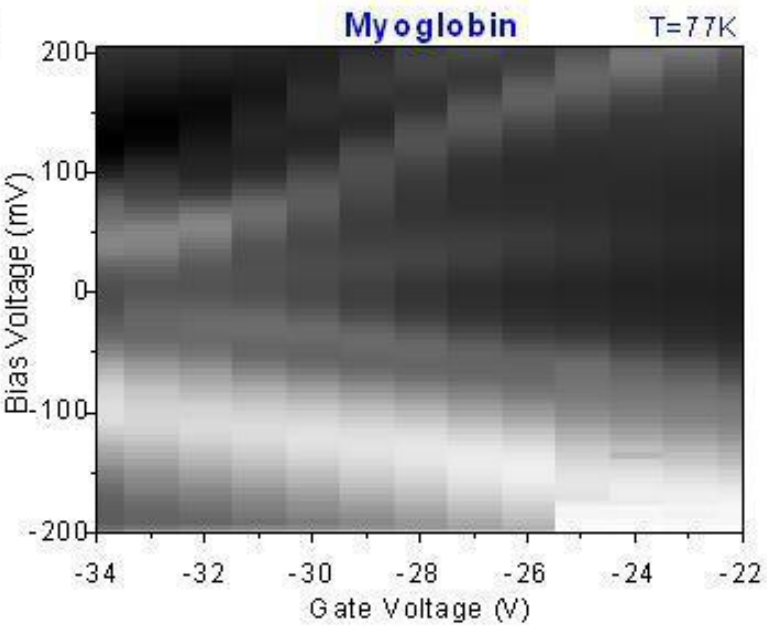

Figure 8.8 One of Mb samples at $77 \mathrm{~K}$ by AC measurement. (a) Some peaks can be found in $d I / d V_{\text {bias }}$ as function of bias voltage and gate voltage; (b)Differential conductance $d I / d V_{\text {bias }}$ as function of gate and bias voltage at $77 \mathrm{~K}$ (gray scale from $-1.5 \mu \mathrm{S}$ [black] to $13.0 \mu \mathrm{S}$ [white]).

\subsection{Some Results at $\sim 5 \mathrm{~K}$}

Another 215 myoglobin samples were measured at $5 \mathrm{~K}$, out of which 73 had conductance peaks. Of these, 21 had conductance peaks that depended on the gate voltage and 21 had a very weak dependence on gate voltage. Figure 8.9(a) shows the result from one sample which has a conductance peak at zero bias that is independent of the gate voltage. Enhanced zero bias conductance was occasionally observed in $\mathrm{Mb}$ samples and some apomyoglobin samples (shown in Fig. 8.9(b)). Kondo effects with gate-independence can also be observed in bare metal break-junctions (Fig. 4.4(d)). ${ }^{47}$ It is impossible to tell the origin of Kondo peak in Fig. 8.9. Furthermore, it is hard to distinguish by applying a magnetic field because the Kondo peak from a metal grain can also be split by a magnetic field. ${ }^{47}$ 
(a)

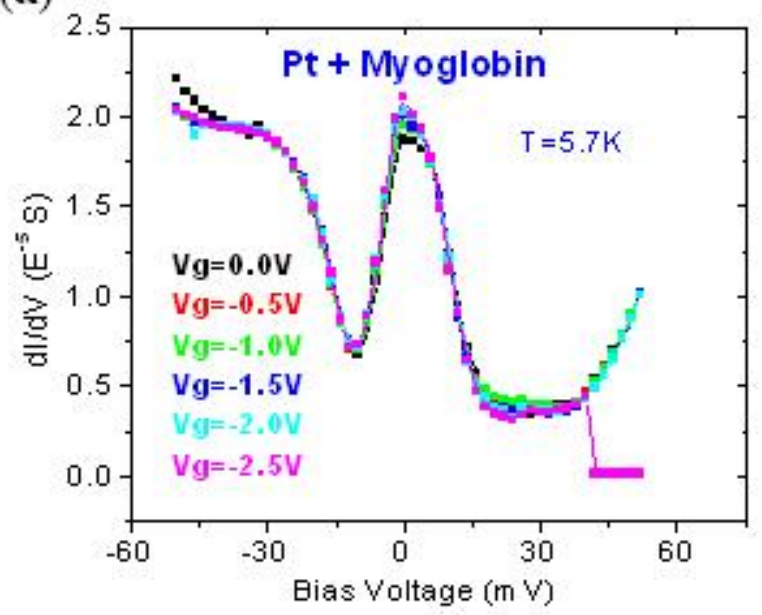

(b)

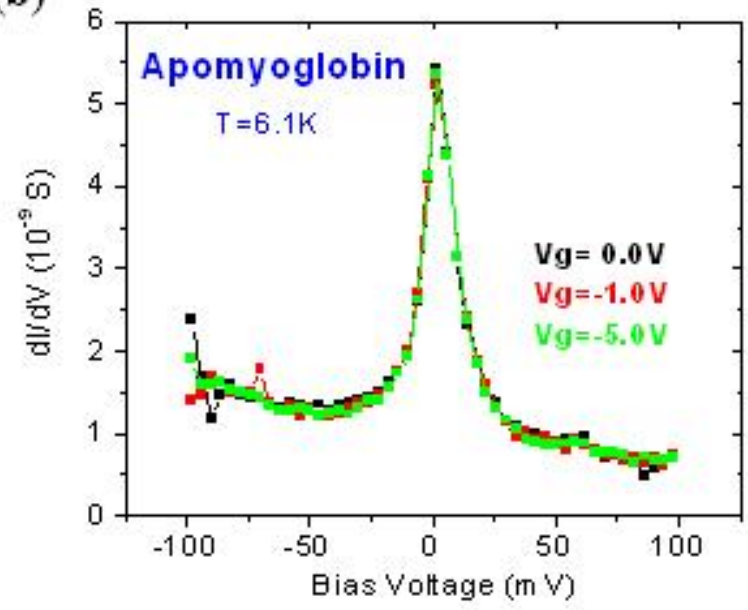

Figure 8.9 Enhanced zero bias conductance was observed in Mb samples (a) and apomyoglobin samples (b). The conductance of peaks are independence of gate voltage.

Figure 8.10 shows the results from one sample whose conductance peaks depended on the gate voltage. Figure 8.10(b) shows that some conductance peaks can be found after breaking the junction (Fig. 8.10(a)). Figure 8.10(c) shows the typical signature of single-electron transistor. The conductance gap keeps opening on the left side of the graph and exceeds $100 \mathrm{mV}$. The conductance on the right side of the graph becomes noisy at gate voltages over $+1.5 \mathrm{~V}$. The crossing potential is at $V_{C}=+30.7 \mathrm{mV}$. $E_{0}$, the electrochemical potential for $\mathrm{Fe}^{2+} / \mathrm{Fe}^{3+}$ transition or redox potential, can be calculated using the equation ${ }^{13}$

$$
V_{C}=\frac{E_{0}}{|e|} \frac{C_{\text {total }}}{C_{G}},
$$

where the total capacitance of the device, $C_{\text {total }}=C_{D}+C_{S}+C_{G}$, with $C_{D}, C_{S}$, and $C_{G}$ being capacitances of the drain, source, and gate contacts, respectively. From Fig. 8.10(c), the positive slope for the resonance line at $V_{G}<0$ and $V<0$ is $C_{G} /\left(C_{G}+C_{D}\right) \approx 0.040$, whereas the negative slope for the resonance line at $V_{G}<0$ and $V>0$ is $-C_{G} / C_{S} \approx-0.025$. Therefore, $C_{\text {total }} / C_{G} \approx 65$ and, using the equation above, $E_{0}=+4.72 \mathrm{meV}$. Figure 8.6(a) is a calculation of the conductivity using a single energy level quantum dot model with these parameters which compares favorably with the experimental data.

In order to determine whether this value is reasonable, we note that previous $\mathrm{CV}$ measurements show that $\mathrm{Mb}$ films are in the ferric state and the electrochemical redox 
potential $E_{0}>0$, in agreement with our result. We also note the appearance of two wide conductance lines that start at $V \sim 5.2 \mathrm{mV}$, indicated by the red arrows in Fig. 8.10(c), that could arise from the vibrational excitation of the protein. Previous spectroscopic studies have shown that a low-frequency mode in myoglobin exists with an energy $\sim 40$ $\mathrm{cm}^{-1}$ ( 4.96 $\mathrm{mV}$ at room temperature). ${ }^{100,101}$ This energy agrees well with the observed resonant line, especially since the peak tends to shift to higher energies at low temperatures. ${ }^{102}$ Therefore we surmise that the wide conductance line is due to electronmolecular vibration coupling.

Another piece of evidence that is consistent with single-protein measurements is the sudden increase in current at $2.0 \mathrm{~V}$ during the junction-breaking process, as shown in Fig. 8.10(a). This is an indication of protein incorporation in the gap of the electrodes, as has been observed previously in junction measurements involving single molecules. ${ }^{24}$ This was not observed in any of our blank Pt junctions. Furthermore, this measurement was made on a self-breaking junction, which could avoid the problem of local heating. 
(a)

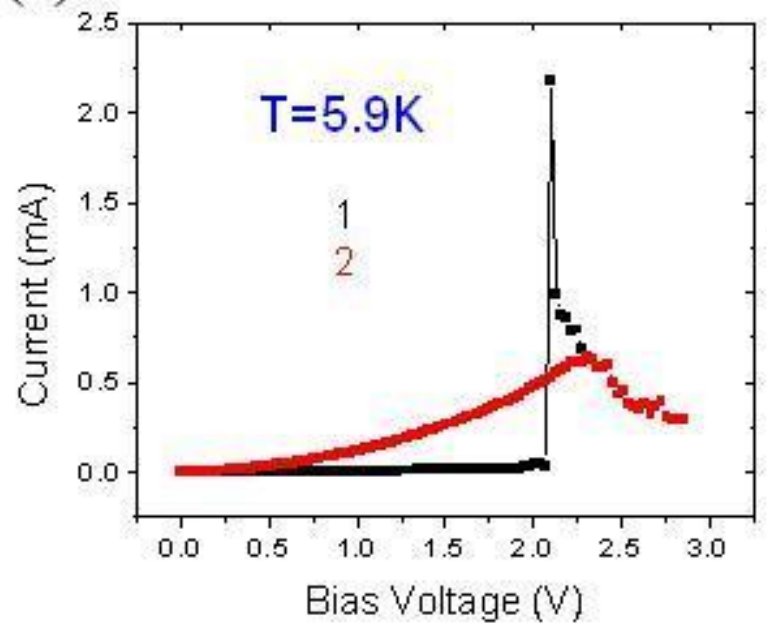

(b)

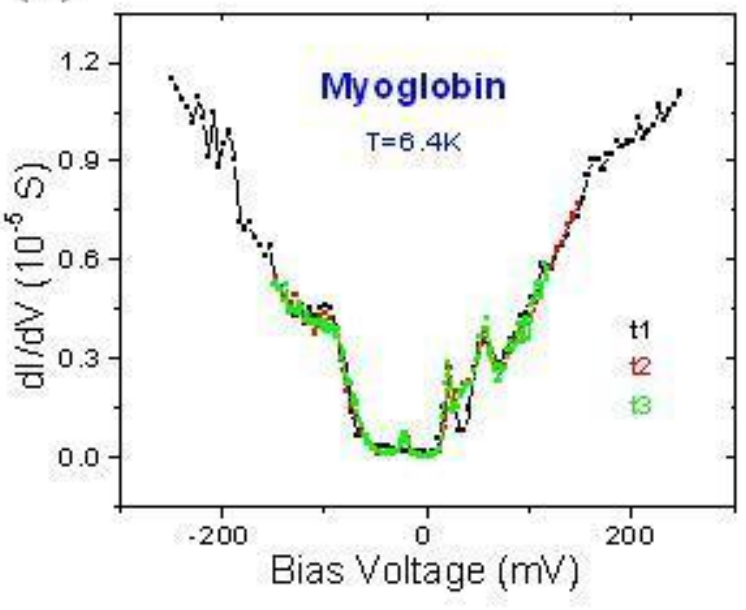

(c)

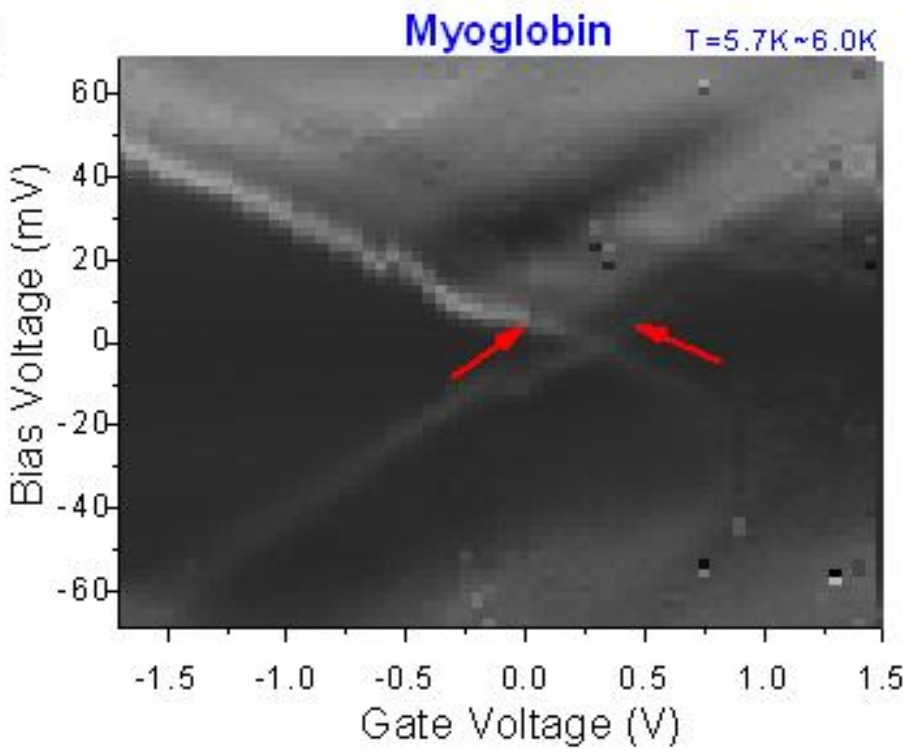

Figure 8.10 One myoglobin sample at $6 \mathrm{~K}$. (a) The current suddenly increase a lot during breaking junction by electromigration; (b) Some conductance peaks can be found; (c) One the typical signature of singleelectron transistor (gray scale from $-1.5 \mu \mathrm{S}$ [black] to $8.2 \mu \mathrm{S}$ [white]).

Another sample with SET behavior is shown in Fig. 8.11. In this case, two deformed Coulomb blockade triangles near zero bias voltage, indicated by the red dots, can be found without a degeneracy point in the differential conductance graph (Fig. 8.11(a)). This means that two charge states do not have the same energy regardless of the gate voltage; indeed, the separation with the top triangles is greater than $30 \mathrm{mV}$. As demonstrated above, this large separation is an indication that the two-step electron transfer process is a viable mechanism. 
In addition, there are three areas that exhibit negative differential conductance (NDC), indicated by three blue arrows, in regions ranging in gate voltage from $-0.7 \mathrm{~V}$ to $0.9 \mathrm{~V}$, from $-0.4 \mathrm{~V}$ to $+1.0 \mathrm{~V}$, and from $+1.7 \mathrm{~V}$ to $+2.2 \mathrm{~V}$. Figure 8.11 (b) shows the conductance near $V_{g}=-0.8 \mathrm{~V}$ and Fig. 8.11(c) shows the $I-V$ measurements taken near $V_{g}$ $=0.2 \mathrm{~V}$, where the NDC is clearly evident. NDC behavior may arise from two quantum dots connected in series. ${ }^{58}$ This suggests that these measurements were the result of transport through two myoglobin proteins in series with each other. Moreover, the existence of several Coulomb triangles is an indication that the multiple-protein complex can have more than two electronically stable states.

The faint line at $V_{\text {bias }}=-25 \mathrm{mV}$ indicated by the green arrow in Fig. 8.11(a) did not change significantly with gate voltage, which indicates electron tunneling by the medium around the heme group. And this peak is also indicated by the green arrow in Fig. 8.11(b).

Finally, we also note that the conductance feature that starts at $V=-97 \mathrm{mV}$, indicated by the red arrow, could arise from one of the excited electronic level of the protein or a vibrational excitation of the protein. In particular, this energy coincides with the energy of the tryptophan amino acid in Mb which is known to have an energy of 760 $\mathrm{cm}^{-1}=94.2 \mathrm{mV}$ (measured at room temperature) ${ }^{75,103}$ The wide conductance line that starts at $V \sim 93 \mathrm{mV}$, indicated by the pink arrow, is another candidate that could arise from the excited state or vibrational excitation of the protein. 
(a)

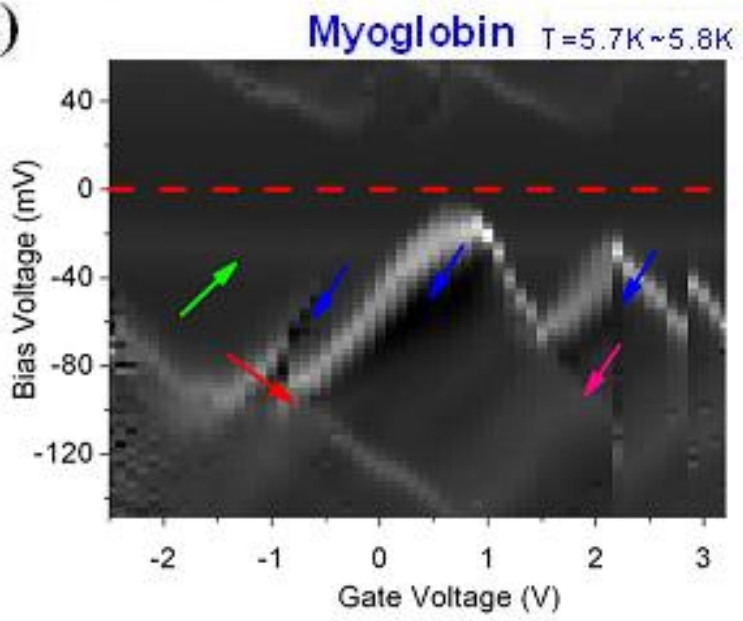

(c)

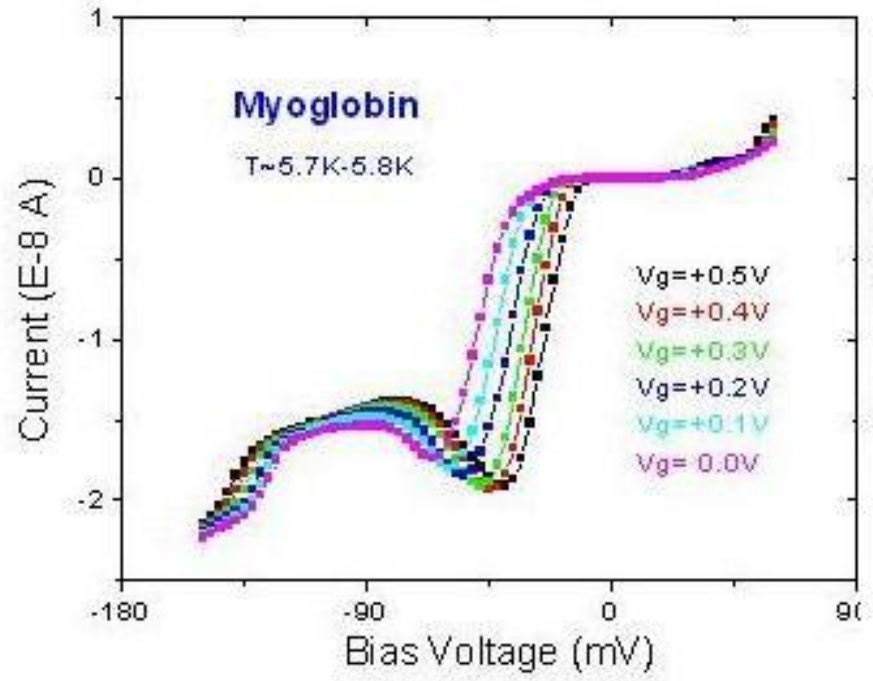

(b)

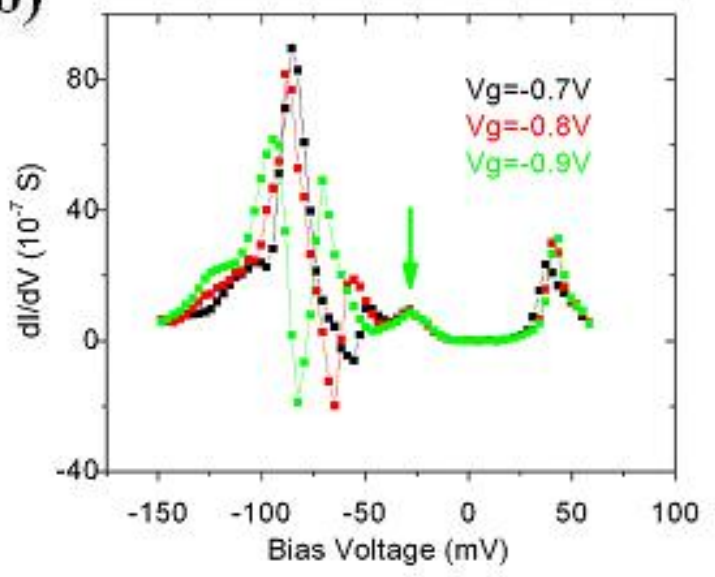

(d)

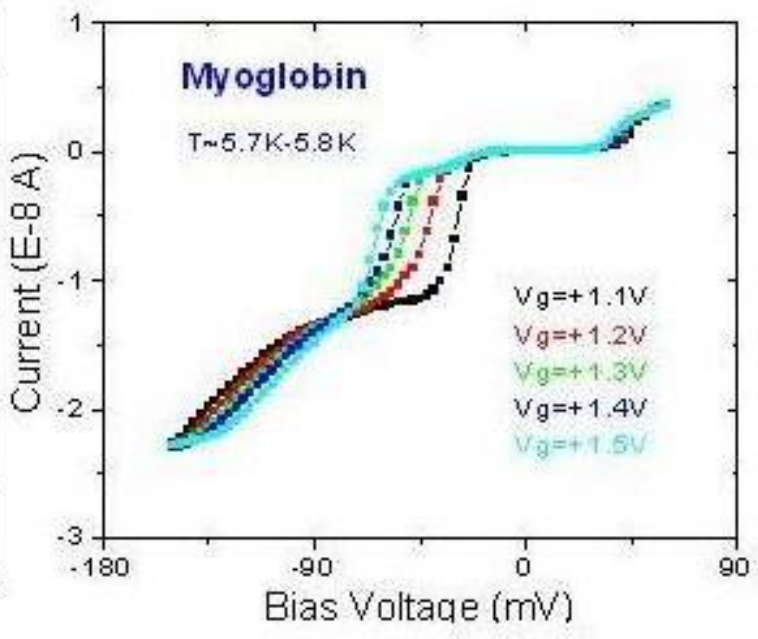

Figure 8.11 One myoglobin sample at $6 \mathrm{~K}$. (a) Differential conductance $d I / d V_{\text {bias }}$ as function of gate and bias voltage at $6 \mathrm{~K}$ (gray scale from $-0.27 \mu \mathrm{S}$ [black] to $1.6 \mu \mathrm{S}$ [white]); (b) Negative differential conductance from $-0.7 \mathrm{~V}$ to $-0.9 \mathrm{~V}$ (gate voltage); (c) The $I-V_{\text {bias }}$ curves change with gate voltage; (d) The $I$ $V_{\text {bias }}$ curves change with gate voltage.

\subsection{Summary}

A weakness of our experiments is that the orientation of myoglobin in the gap of electrodes is essentially unknown, and the electron transfer pathway is very important for single electron transport by single proteins. Therefore, different protein orientations may result in different electron transfer pathways and different protein relaxation times. This may explain why a two-step process was observed in some of our samples and not in 
others. The mechanism of electron transfer by the protein in nanometer-gap electrodes could be explained simply by the relaxation time. Our experimental results show that fast protein relaxation is the condition for two-step SET behavior.

In summary, we have immobilized myoglobin protein between nanometer gap electrodes and have observed single electron transistor behavior that may be associated with the iron ion redox reaction. In particular, indications of resonant tunneling and two step transfer processes were identified. 


\section{Chapter 9}

\section{Conclusions}

The electronic properties of myoglobin proteins with ordinary transistor geometry at cryogenic temperatures were studied in order to provide more detailed information for the electron transfer by protein. To measure the proteins ( $\sim 4 \mathrm{~nm}$ in diameter), an electromigration technique using Pt instead of Au was employed to fabricate electrodes with nanometer-sized gaps in order to create single electron transistors based on myoglobin proteins. A significant effort was made to check for artifacts by measuring blank junctions and junctions with apomyoglobin and demonstrating that the protein's structure remains unchanged after cooling to cryogenic temperatures. The appearance of $\mathrm{Au}$ or Pt grains with nanometer-sized in the gap of the electrodes after breaking the junction was a problem because the electrical properties of small islands display SET behavior.

For the immobilized myoglobin proteins, single electron transistor behavior that may be associated with the iron ion redox reaction was observed. It is well-known that the heme group is not at the center of myoglobin protein, and that the conformation and orientation of the proteins can not be controlled through the processes of protein deposition and electromigration. Therefore, the asymmetrical structure and numerous orientation configurations of the protein could significantly affect the conductance measurements. One method used to identify conduction by the protein is to look for conductance enhanced by vibrational excitations. When an electron tunnels on to or off from a molecule, the molecular equilibrium configuration undergoes a certain change that also reflects the change in the local electrostatic environment. Due to variations in the local environment of each single molecule device, the observed excitation spectrum usually differed from device to device.

In this work, the mechanism of electron transfer by myoglobin using nanometergap platinum electrodes at cryogenic temperatures was investigated. The experimental results suggest single electron transport behavior is mediated by resonance of the electronic levels of the heme group in the myoglobin protein. This demonstrates that 
myoglobin across nanometer-gap electrodes could be utilized to fabricate single electron transistors. In particular, indications of resonant tunneling and two step transfer processes were identified. 


\section{Bibliography}

1. R. A. Marcus and N. Sutin, Biochim. Biophys. Acta, 811, 265 (1985).

2. D.S. Bendall, Protein Electron Transfer (BIOS Publishers, Oxford, 1996).

3. N. J. Tao, Phys. Rev. Lett., 76, 4066 (1996).

4. A. Brajter-Toth and J. Q. Chambers, Electroanalytical Methods for Biological Materials (Marcel Dekker, New York, 2002).

5. R. H. Garrett and C. M. Grisham, Biochemistry (Harcourt Brace College Publishers, 1999), p480, p. 481, p. 114.

6. R. S. Drago, Physical Methods in Inorganic Chemistry (New York: Reinhold Publishing Corporation, 1965).

7. B. N. Flggis and M. A. Hitchman, Ligand Field Theory and Its Applications (Wiley$\mathrm{VCH}, 2000)$.

8. H. Wang, G. Peng, L. M. Miller, E. M. Scheuring, S. J. George, M. R. Chance, and S.

P. Cramer, J. Am. Chem. Soc. 119, 4921 (1997).

9. M. W. Makinen, A. K. Churg, and H. A. Glick, Proc. Natl. Acad. Sci. USA 75, 2291 (1978).

10. D. Segal, Ph.D. Dissertation, Tel-Aviv University, 2003.

11. A. J. Bard \& L. R. Faulkner, Electrochemical Methods: Fundamentals and Applications (New York: John Wiley \& Sons, 2nd Edition, 2000).

12. R. S. Nicholson \& I. Shain, Anal. Chem. 36, 706 (1964).

13. J. Park, Ph.D. Dissertation, University of California-Berkeley, 2003.

14. A. Aviram and M. A. Ratner, Chem. Phys. Lett. 29, 277 (1974).

15. A. Alessandrini, S. Corni and P. Facci, Phys. Chem. Chem. Phys. 8, 4383 (2006).

16. J. Zhang, Q. Chi, A. M. Kuznetsov, A. G. Hansen, H. Wackerbarth, H. E. M. Christensen, J. E. T. Andersen and J. Ulstrup, J. Phys. Chem. B 106, 1131 (2002).

17. Q. Chi, O. Farver, and J. Ulstrup, Proc. Natl. Acad. Sci. USA. 102, 16203 (2005).

18. T. Albrecht, A. Guckian, J. Ulstrup, and J. G. Vos, Nano Lett., 5, 1451 (2005).

19. D. DeVault and B. Chance, Biophys. J. 6, 825 (1966).

20. D. DeVault, Q. Rev. Biophys. 13, 387 (1980).

21. C. W. J. Beenakker, Phys. Rev. B 44, 1646 (1991). 
22. L. L. Sohn, L. P. Kouwenhoven, and G. Schön, Mesoscopic Electron Transport (Kluwer Academic Publishers, Dordrecht, 1997).

23. L. H. Yu, Ph.D. Dissertation, Rice University, 2006.

24. J. Park, A. N. Pasupathy, J. I. Goldsmith, C. Chang, Y. Yaish, J. R. Petta, M. Rinkoski, J. P. Sethna, H. D. Abruna, P. L. McEuen and D. C. Ralph, Nature, 417, 722 (2002).

25. S. Kubatkin, A. Danilov, M. Hjort, J. Cornil, J.-L. Brédas, N. Stuhr-Hansen, P. Hedegård and T. Bjørnholm, Nature, 425, 698 (2003).

26. L. H. Yu, Z. K. Keane, J. W. Ciszek, L. Cheng, M.P. Stewart, J. M. Tour, and D. Natelson, Phys. Rev. Lett. 93, 266802 (2004).

27. R. C. Jaklevic and J. Lambe, Phys. Rev. Lett. 17, 1139 (1966).

28. J. Lambe and R. C. Jaklevic, Phys. Rev. 165, 821 (1968).

29. C. J. Adkins and W. A. Phillips, J. Phys. C. 18, 1313 (1985).

30. W. Ho, J. of Chem. Phys. 117, 11033 (2002).

31. W. Wang, Ph.D. Dissertation, Yale University, 2004.

32. M G. Simonsen and R. V. Coleman, Nature 244, 218 (1973).

33. Y. Glazer and M. Gitterman, Phys. Rev. B 43, 1855 (1991).

34. D. Bohm, Quantum Theory, (Prentice-Hall, New York, 1951).

35. E. Merzbacher, Quantum Mechanics, (John Wiley \& Sons, Inc., 1970).

36. E. Bonet, M. M. Deshmukh, and D. C. Ralph, Phys. Rev. B 65, 045317-1 (2002).

37. C. Kittel, Introduction to Solid State Physics, (John Wiley \& Sons, Inc., 1996), p628, p542.

38. J. Kondo, Progress of Theoretical Physics, 32, 37 (1964).

39. P.W. Anderson, Phys. Rev. 124, 41 (1961).

40. L. Kouwenhoven and L. Glazman, Phys. World, 14, 33 (2001).

41. N. J. Tao, Nature Nanotechnology 1, 173 (2006).

42. R. H. M. Smit, Y. Noat, C. Untiedt, N. D. Lang, M. C. van Hemert and J. M. van Ruitenbeek, Nature 419, 906 (2002).

43. A. F. Morpurgo, C.M. Marcus, and D.B. Robinson, Appl. Phys. Lett. 74, 2084 (1999).

44. J. Chen, M. A. Reed, A. M. Rawlett, and J. M. Tour, Science 286, 1550 (1999). 
45. H. Park, A. K. L. Lim, J. Park, A. P. Alivisatos, and P. L. McEuen, Appl. Phys. Lett. 75, 301 (1999).

46. P. Rai-Choudhury, Handbook of Microlithography, Micromachining, and Microfabrication. Volume 1: Microlithography (SPIE Press Monograph Vol. PM39, 1997).

47. A. A. Houck, J, Labaziewicz, E. K. Chan, J. A. Folk, and I. L. Chuang, Nano Letters 5, 1685 (2005).

48. R. Sordan, K. Balasubramanian, M. Burghard and K. Kern, Appl. Phys. Lett. 87, 013106 (2005).

49. H. S. J. van der Zant, Y.-V. Kervennic, M. Poot, K. O'Neill, Z. de Groot, H. B. Heersche, N. Stuhr-Hansen, T. Bjørnholm, D. Vanmaekelbergh, C. A. van Walree, and L. W. Jenneskens, Faraday Discuss. 131, 347 (2006).

50. L. L. Sohn, L. P. Kouwenhoven, and G. Schön, Mesoscopic Electron Transport (Kluwer Academic Publishers, Dordrecht, 1997).

51. S. Kubatkin, A. Danilov, M. Hjort, J. Cornil, J.-L. Brédas, N. Stuhr-Hansen, P. Hedegård and T. Bjørnholm, Nature, 425, 698 (2003).

52. K. K. Likharev, Proc. IEEE 87, 606 (1999).

53. A. V. Danilov, D. S. Golubev, and S. E. Kubatkin, Phys. Rev. B 65, 125312 (2002).

54. A. V. Danilov, S. E. Kubatkin, S. G. Kafanov and T. Bjørnholm, Faraday Discuss. 131, 337 (2006).

55. S. E. Kubatkin, A. V. Danilov, H. Olin and T. Cleason, J. Low Temp. Phys. 118, 307 (2000).

56. F. R. Waugh, M. J. Berry, D. J. Mar, R. M. Westervelt, K. L. Campman, and A. C. Gossard, Phys. Rev. Lett. 75, 705 (1995).

57. C. Livermore, C. H. Crouch, R. M. Westervelt, K. L. Campman, and A. C. Gossard, Science 274, 1332 (1996).

58. J. Fransson and O. Eriksson, J. Phys.: Condens. Matter 16, L85 (2004).

59. G. Esen and M. S. Fuhrer, Appl. Phys. Lett. 87, 263101 (2005).

60. T. Taychatanapat, K. I. Bolotin, F. Kuemmeth, and D. C. Ralph, Nano Letters 7, 652 (2007).

61. D. R. Ward, N. J. Halas and D. Natelson, Appl. Phys. Lett. 93, 213108 (2008). 
62. F. Kuemmeth, K. I. Bolotin, S. Shi, and D. C. Ralph, Nano Letters 8, 4506 (2008).

63. C. S. Wu, C. D. Chen, S. M. Shih and W. F. Su, Appl. Phys. Lett. 81, 4595 (2002).

64. Y. V. Kervennic, H. S. J. Van der Zant, A. F. Morpurgo, L. Gurevich and L. P. Kouwenhoven, Appl. Phys. Lett. 80, 321 (2002).

65. U. Bilitewski, Anal. Chim. Acta 568, 232 (2006).

66. F. Rusmini, Z. Zhong, and J. Feijen, Biomacromolecules 8, 1775 (2007).

67. D. Hobara, Y. Uno, and T. Kakiuchi, Phys. Chem. Chem. Phys. 3, 3437 (2001).

68. Z. Li, S.-C. Chang, and R. S. Williams, Langmuir 19, 6744 (2003).

69. P. Gemeiner, In Enzyme Engineering: Immobilized Biosystems (Ellis Horwood Limited, 1992).

70. R. F. Taylor, Protein Immobilization: Fundamentals and Applications (Marcel

Dekker, Inc., 1991).

71. H. Park, J. Park, A. K. L. Lim, E. H. Anderson, A. P. Alivisatos and P. L. McEuen, Nature 407, 57 (2000).

72. E. B. Wilson, J. C. Decius and P. C. Cross, Molecular vibrations (McGraw-Hill, 1955).

73. A. P. Arya, Fundamentals of Atomic Physics (Allyn and Bacon, Inc., 1971).

74. T. G. Spiro, T. C. Strekas, J. Am. Chem. Soc. 96, 338 (1974).

75. M. Feng and T. Hiroyasu, J. Am. Chem. Soc. 123, 3013 (2001).

76. X. Xiao, B. Xu and N. J. Tao, J. Am. Chem. Soc. 126, 5370 (2004).

77. C. Shih, A. K. Museth, M. Abrahamsson, A. M. Blanco-Rodriguez, A. J. Di Bilio, J. Sudhamsu, B. R. Crane, K. L. Ronayne, M. Towrie, A. Vlěek, Jr., J. H. Richards, J. R. Winkler, H. B. Gray, Science, 320, 1760 (2008).

78. J. M. Bollinger Jr., Science, 320, 1730 (2008).

79. A. Salomon, D. Cahen, S. Lindsay, J. Tomfohr, V. B. Engelkes, and C. D. Frisbie, Adv. Mater. 15, 1881 (2003).

80. R. Tsu and L. Esaki, Appl. Phys. Lett. 22, 562 (1973).

81. R. Landauer, IBM J. Res. Dev. 1, 223 (1957).

82. R. Landauer, J. Phys.: Condens. Matter 1, 8099 (1989).

83. R. Landauer, Philos. Mag. 21, 863 (1970). 
84. P. W. Anderson, D. J. Thouless, E. Abrahams and D. S. Fisher, Phys. Rev. B 22, 3519 (1980).

85. E. N. Economou and C. M. Soukoulis, Phys. Rev. Lett 46, 618 (1981).

86. H.-L. Engquist and P. W. Anderson, Phys. Rev. B 24, 1151 (1981).

87. O. M. Auslaender, A. Yacoby, R. de Picciotto, K. W. Baldwin, L. N. Pfeiffer and K. W. West, Phys. Rev. Lett. 84, 1764 (2000).

88. A. B. Fowler, G. L. Timp, J. J. Wainer, and R. A. Webb, Phys. Rev. Lett. 57, 138 (1986).

89. M. Ya. Azbel, A. Hartstein and D. P. DiVincenzo, Phys. Rev. Lett. 52, 1641 (1984).

90. J. C. Kendrew, G. Bodo, H. M. Dintzis, R. G. Parrish, H. Wyckoff, and D. C. Phillips, Nature 181, 662 (1958).

91. J. F. Rusling, Acc. Chem. Res., 31, 363 (1998).

92. A.-E. F. Nassar, Z. Zhang, N. Hu, J. F. Rusling, and T. F. Kumosinski, J. Phys. Chem. $B$ 101, 2224 (1997).

93. K. M. Faulkner, C. Bonaventura, and A. L. Crumbliss, J. Biol. Chem. 270, 13604 (1995).

94. G. R. Moore, G. W. Pettigrew, and N. K. Rogers, Proc. Natl. Acad. Sci. U.S.A. 83, 4998 (1986).

95. D. C. Rees, Proc. Natl. Acad. Sci. U.S.A. 82, 3082 (1985).

96. M. Ratner, Nature 435, 575 (2005).

97. J. Zhang, Q. Chi, T. Albrecht, A. M. Kuznetsov, M. Grubb, A. G. Hansen, H. Wackerbarth, A. C. Welinder and J. Ulstrup, Electrochimica Acta 50, 3143 (2005).

98. F. A. Tezcan, J. R.Winkler, and H. B.Gray, J. Am. Chem. Soc. 120, 13383 (1998).

99. D. A. Cherepanov, L. I. Krishtalik, and A. Y. Mulkidjanian, Biophys. J. 80, 1033 (2001).

100. F. Rosca, A. T. N. Kumar, X. Ye, T. Sjodin, A. A. Demidov and P. M. Champion, J. Phy. Chem. A 104, 4280 (2000).

101. F. Gruia, M. Kubo, X. Ye, and P. M. Champion, Biophysical Journal 94, 2252 (2008).

102. M. Korostishevsky, Z. Zaslavsky and S. S. Stavrov, Biophys. J. 86, 656 (2004). 
103. V. L. Tilly, O. Sire, B. Alpert, L. Chinsky and P. Y. Turpin, Biochemistry 30, 7248 (1991).

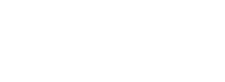

